Supporting Information for

\title{
A mechanism-based sphingosine-1-phosphate lyase inhibitor
}

Guillem Pons, ${ }^{1}$ Daniel Riba, ${ }^{1}$ Mireia Casasampere, ${ }^{1,2}$ Eduardo Izquierdo, ${ }^{1,2}$ José-Luís Abad, ${ }^{1}$ Gemma Fabriàs, ${ }^{1,3}$ Pilar G. Rodríguez Ortega, ${ }^{4}$ Juan J. López-González, ${ }^{4}$ Manuel Montejo, ${ }^{4}$ Josefina Casas, ${ }^{* 1,3}$ and Antonio Delgado*1,2

\section{Index}

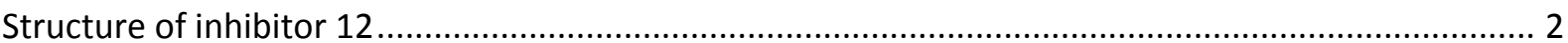

Computational details and conformational search results for $6 \mathrm{a}$ and $6 \mathrm{~b}$ species................................ 2

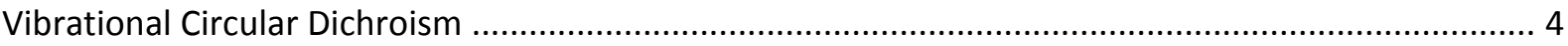

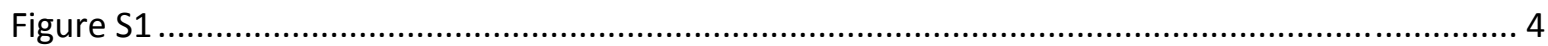

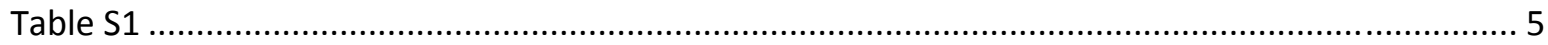

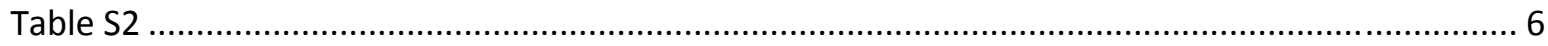

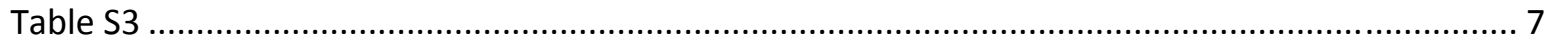

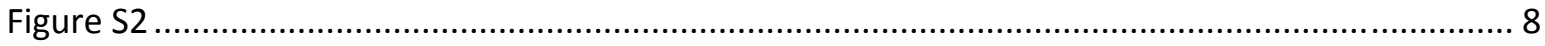

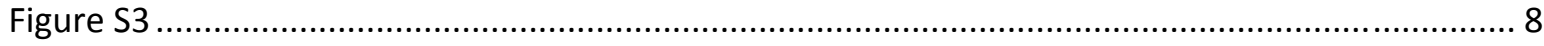

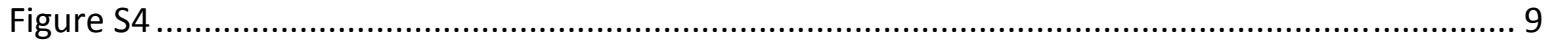

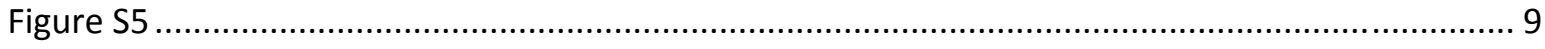

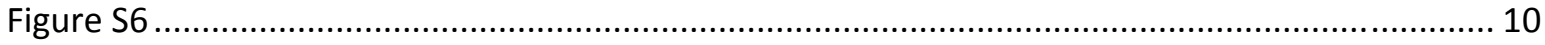

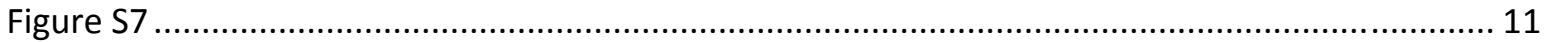

Cartesian Coordinates matrices of the optimized (PCM/M062X/cc-pVTZ) structures of the set of

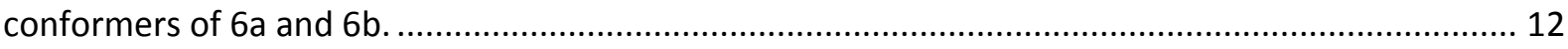

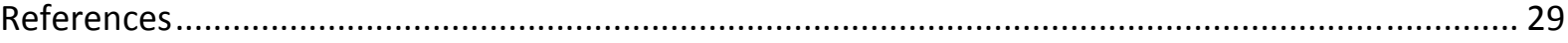

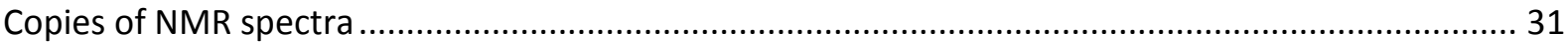




\section{Structure of inhibitor 12}

The hS1PL inhibitor 12 was a donation from Novartis (see ref ${ }^{1}$ )

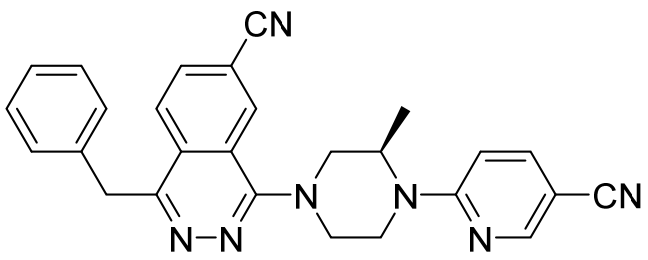

12

\section{Computational details and conformational search results for $6 \mathrm{a}$ and $6 \mathrm{~b}$ species.}

A scheme of the workflow in the conformational search and the definition of the theoretical model is shown in Figure S1. A Montecarlo conformational search has been performed over the structures of $\mathbf{6 a}$ and $\mathbf{6 b}$ with the MMFF force field as implemented in Spartan08 program package. ${ }^{2}$ The selected nonredundant structures were subsequently optimized and confirmed as minima in the potential energy surfaces (PES) of the systems by computation of their harmonic vibrational frequencies with the $\mathrm{B} 3 \mathrm{LYP}^{3,4}$ and $\mathrm{M} 062 \mathrm{X}^{5}$ functionals in conjunction with the $6-31+\mathrm{G}^{*}$ basis set. ${ }^{6,7}$ Those conformers lying in an energy threshold of $3 \mathrm{kcal} \mathrm{mol}^{-1}$ were further optimized to consider the effect of the solvent in their structures by applying integral equation formalism (IEF) version of the continuum PCM solvation model ${ }^{8-12}$ using the dielectric constant value corresponding to $\mathrm{CCl}_{4}$. In these calculations, the basis set used was the so-called cc-pVTZ. ${ }^{13}$ The final set of minima, those representing $98 \%$ of the Boltzmann population of the total conformational sample composition at the PCM/M062X/cc-pVTZ, were re-optimized at PCM/B3LYP/cc-pVTZ at which level the harmonic vibrational IR and VCD spectra were calculated. No imaginary vibrational frequencies were calculated for any of the structures in the final set of conformers. Their calculated Gibbs free energies $(\mathrm{H})$ along with the relative values of this magnitude referred to the global minima (kcal mol-1) and the subsequently calculated Boltzmann populations are reported in Table S1). The cartesian coordinates matrices of the 17 structures ( 9 for $6 \mathrm{a}$ and 8 for $6 \mathrm{~b}$ ) used for the theoretical estimation of the vibrational spectra are also reported as part of the Supplementary Material. DFT calculations were performed according to the methods related above as implemented in the Gaussian09 program package. ${ }^{14}$. Geometry optimization criteria were SCF=tight and Int=Ultrafine.

The results of the exploration of the potential energy surfaces of $\mathbf{6 a}$ and $\mathbf{6 b}$ point out that the global minima for both species in CCl4 solution (PCM/M062x/cc-pVTZ) are characterized by 
the linear (all trans) arrangement of the side alkyl chain and the syn-periplanar orientation of the vinyl group towards the endocyclic $C_{3}-O$ bond $\left(\phi_{C=C C_{3} O} \approx 0^{\circ}\right)$, suggesting the possible incidence of an intramolecular hydrogen bond $\left(d_{O \ldots H}=2,45 \AA\right)$. Calculated Boltzmann's populations for these structures are $35,8 \%$ and $47,4 \%$ for $\mathbf{6 a}$ and $\mathbf{6 b}$, respectively (Table S1, Figure S2). For both diastereomers, the sets of local minima include both structures with folded alkyl chains and syn-periplanar vinyl arrangements and with linear alkyl chains and antiperiplanar arrangements of the vinyl moiety.

It is well known that, although the linear arrangement of alkyl chains is more energetically favorable, in long enough chains intramolecular dispersion forces may cause attractive intramolecular interactions that promote the occurring of folded conformations. ${ }^{15,16}$ It is also known that B3LYP hybrid functional does not account for long range dispersion interactions, ${ }^{17}$ which may justify the observed B3LYP's overestimation of the contribution of structures with linear side chains to the total composition of the conformational mixture (Table S1). In contrast, dispersion corrected Thrular's M062x functional ${ }^{5}$ allows a finer description of the conformational landscape of both systems. Therefore, we have used Boltzmann's populations calculated with the M062x functional to balance the individual contributions of each conformer to the overall spectral IR and VCD profiles calculated for $6 a$ and $6 \mathrm{~b}$ with the B3LYP method, that is well-known to have proven efficiency in the estimation of the VCD signals intensity and signs.

Following this methodology, theoretical IR and VCD spectra were built using GaussView6. ${ }^{18}$ FWHMs of the bands were set to $8 \mathrm{~cm}^{-1}$. Calculated harmonic vibrational wavenumbers were scaled by $0.977(\mathbf{6 a})$ and $0.980(\mathbf{6 b})$ to maximize the similarity between experimental and theoretical profiles according to the statistical validation of the absolute configuration performed by CompareVOA. ${ }^{19,20}$ The use of this scale factor is justified considering the recommended value of $0.965 .^{21}$ The correctness of the approximation proposed can be inferred from the theoretically supported interpretation of the IR and VCD spectra of both diastereomers in the $1400-1000 \mathrm{~cm}^{-1}$ region (see Figure 1 in the manuscript). The complete lists of vibrational modes calculated in this region for the two main conformers of $\mathbf{6 a}$ and $\mathbf{6 b}$ are also reported as Supporting Information in Tables S2 and S3. 


\section{Vibrational Circular Dichroism}

The experimental IR and VCD spectra of $\mathbf{6 a}$ and $\mathbf{6 b}$ and their respective enantiomers are reported in Figures S3-S5. For the recording of the spectra, fixed pathlength cuvettes $(100 \mathrm{~mm})$ equipped with $\mathrm{BaF}_{2}$ windows were used. Concentrations were optimized to maximize IR absorbance considering the limitations derived by the availability of samples. VCD spectra were recorded using a Bruker PMA50 optical bench coupled to a Vertex 70 FT-IR spectrometer. In the PMA50, the infrared radiation is focused by a $\mathrm{BaF}_{2}$ lens, passing an optical filter (3800$600 \mathrm{~cm}^{-1}$ range) and a ZnSe photo-elastic modulator (PEM $50 \mathrm{kHz}$ ). The light beam is finally collected by a D313/QMTC detector with non-dichroic $\mathrm{BaF}_{2}$ windows. A previous calibration of the PEM at a fixed wavenumber is required before recording a VCD spectrum. Calibration at $1250 \mathrm{~cm}^{-1}$ allowed us to obtain a VCD signal over the whole infrared region of interest, 1400$1000 \mathrm{~cm}^{-1}$. Every VCD spectrum was the result of averaging a minimum of 50000 scans $(18 \mathrm{~h}$ acquisition time) at a spectral resolution $4 \mathrm{~cm}^{-1}$. After data acquisition, individual baselines of the VCD spectra were corrected according to the standard methodology. ${ }^{22}$ VCD profiles were also smoothed using a 7 points Savitzky-Golay algorithm.

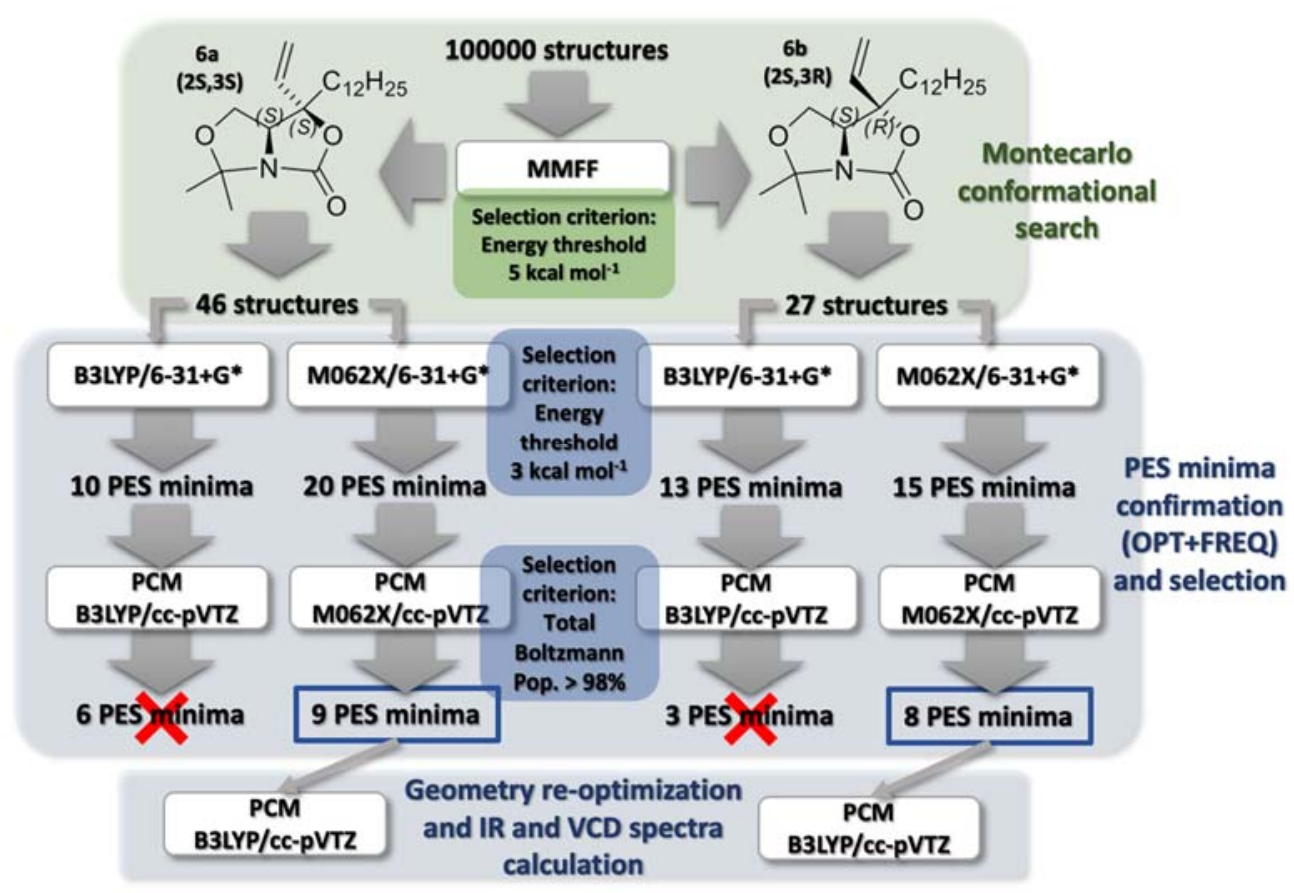

Figure S1. Schematic view of the workflow in the conformational search and definition of the theoretical models 
Table S1. Absolute values of $\mathbf{G}(\mathbf{H}), \Delta \mathrm{G}\left(\mathrm{kcal} \mathrm{mol}^{-1}\right)$ and Boltzmann populations (\%) for the set of conformers of $\mathbf{6 a}$ and $\mathbf{6 b}$. $^{*}$

\begin{tabular}{|c|c|c|c|c|c|c|c|}
\hline Conf. & $\mathbf{G}(\mathbf{H})$ & $\begin{array}{c}\Delta G \\
\left(\mathrm{kcal} \mathrm{mol}^{-1}\right)\end{array}$ & $\begin{array}{c}\text { Boltzmann } \\
\text { Pop.(\%) }\end{array}$ & Conformer & G (H) & $\begin{array}{c}\Delta G \\
\left(\mathrm{kcal} \mathrm{mol}^{-1}\right)\end{array}$ & $\begin{array}{c}\text { Boltzmann } \\
\text { Pop. (\%) }\end{array}$ \\
\hline & \multicolumn{3}{|c|}{ PCM / M062X / cc-pVTZ } & & \multicolumn{3}{|c|}{ PCM / B3LYP / cc-pVTZ } \\
\hline & & \multicolumn{6}{|c|}{$6 a(2 S, 3 S)$} \\
\hline $6 a-1$ & -1102.374133 & 0.00 & 35.8 & $6 a-1$ & -1102.872516 & 0.00 & 91.6 \\
\hline $6 a-2$ & -1102.373455 & 0.43 & 17.5 & $6 a-9$ & -1102.869127 & 2.13 & 2.5 \\
\hline $6 a-3$ & -1102.373301 & 0.52 & 14.8 & $6 a-3$ & -1102.868970 & 2.23 & 2.1 \\
\hline $6 a-4$ & -1102.373124 & 0.63 & 12.3 & $6 a-2$ & -1102.868900 & 2.27 & 2.0 \\
\hline $6 a-5$ & -1102.372363 & 1.11 & 5.5 & $6 a-4$ & -1102.868220 & 2.70 & 1.0 \\
\hline $6 a-6$ & -1102.371998 & 1.34 & 3.7 & $6 a-10$ & -1102.867975 & 2.85 & 0.7 \\
\hline $6 a-7$ & -1102.371909 & 1.40 & 3.4 & & & & \\
\hline $6 a-8$ & -1102.371795 & 1.47 & 3.0 & & & & \\
\hline \multirow[t]{2}{*}{ 6a-9 } & -1102.371766 & 1.49 & 2.9 & & & & \\
\hline & & \multicolumn{6}{|c|}{$6 b(2 S, 3 R)$} \\
\hline $6 b-1$ & -1102.375857 & 0.00 & 47.4 & $6 b-1$ & -1102.874236 & 0.00 & 90.1 \\
\hline $6 b-2$ & -1102.375171 & 0.43 & 22.9 & $6 b-2$ & -1102.871553 & 1.68 & 5.3 \\
\hline $6 b-3$ & -1102.374537 & 0.83 & 11.7 & $6 b-4$ & -1102.871446 & 1.75 & 4.7 \\
\hline $6 b-4$ & -1102.374289 & 0.98 & 9.0 & & & & \\
\hline $6 b-5$ & -1102.373207 & 1.66 & 2.9 & & & & \\
\hline $6 b-6$ & -1102.372930 & 1.84 & 2.1 & & & & \\
\hline $6 b-7$ & -1102.372858 & 1.88 & 2.0 & & & & \\
\hline $6 b-8$ & -1102.372856 & 1.88 & 2.0 & & & & \\
\hline
\end{tabular}

${ }^{*}$ No imaginary frequencies were calculated for any of the structures, confirming them as real minima of the PES 
Table S2. PCM/B3LYP/cc-pVTZ calculated harmonic wavenumbers in the region of interest for the main conformer of $\mathbf{6 a}(6 \mathrm{a}-1)$.

\begin{tabular}{|c|c|c|c|c|c|c|}
\hline $\begin{array}{l}\text { Normal } \\
\text { mode }\end{array}$ & $\begin{array}{c}\omega \\
\left(\mathrm{cm}^{-1}\right)\end{array}$ & $\begin{array}{l}\omega_{\text {scaled }} \\
\left(\mathrm{cm}^{-1}\right)^{*}\end{array}$ & $\begin{array}{c}\mathbf{I R} \\
\text { intensity }\end{array}$ & $\begin{array}{c}\text { Rotor } \\
\text { strength }\end{array}$ & $\begin{array}{c}\mathrm{I}-\mathrm{M} \\
\text { angle }\end{array}$ & Description** \\
\hline 122 & 1422 & 1392 & 17 & 2 & 89 & $\delta_{\mathrm{CH}_{2}(\text { ring })}$ \\
\hline 121 & 1413 & 1383 & 2 & 0 & 90 & $\delta_{\mathrm{CH}_{3} \text { (chain) }}$ \\
\hline 120 & 1412 & 1382 & 27 & 22 & 80 & $\delta_{\mathrm{CH}_{2}(\text { ring })}+\delta_{\mathrm{HC}_{2}-\text { ring }}$ \\
\hline 119 & 1407 & 1377 & 25 & -11 & 94 & $\delta_{\mathrm{CH}_{2}(\text { ring })}$ \\
\hline 118 & 1405 & 1376 & 8 & 2 & 82 & $\delta_{\mathrm{CH}_{2} \text { (chain) }}$ \\
\hline 117 & 1402 & 1373 & 1 & -2 & 98 & $\delta_{\mathrm{CH}_{2} \text { (chain) }}$ \\
\hline 116 & 1400 & 1371 & 5 & 7 & 81 & $\delta_{\mathrm{CH}_{2} \text { (chain) }}$ \\
\hline 115 & 1398 & 1369 & 2 & -4 & 95 & $\delta_{\mathrm{CH}_{2} \text { (chain) }}$ \\
\hline 114 & 1391 & 1362 & 5 & -1 & 93 & $\delta_{\mathrm{CH}_{2} \text { (chain) }}$ \\
\hline 113 & 1374 & 1346 & 37 & -15 & 95 & $\delta_{\left.\mathrm{CH}_{2} \text { (chain) }\right)}+\delta_{\mathrm{HC}_{2}-\text { ring }}$ \\
\hline 112 & 1367 & 1338 & 77 & -41 & 96 & $\delta_{\left.\mathrm{CH}_{2} \text { (chain) }\right)}+\delta_{H C_{2}-\text { ring }}$ \\
\hline 111 & 1352 & 1324 & 4 & 2 & 86 & $\delta_{\mathrm{CH}_{2}(\text { chain })}+\delta_{\mathrm{HC}_{2}-\text { ring }}+\delta_{\mathrm{CH}_{2}(\text { ring })}$ \\
\hline 110 & 1351 & 1323 & 10 & 2 & 60 & $\delta_{\left.\mathrm{CH}_{2} \text { (chain }\right)}+\delta_{\mathrm{HC}_{2}-\text { ring }}+\delta_{\mathrm{CH}_{2}(\text { ring })}$ \\
\hline 109 & 1343 & 1315 & 11 & -22 & 104 & $\delta_{\left.\mathrm{CH}_{2} \text { (chain) }\right)}+\delta_{\mathrm{HC}_{2}-\text { ring }}+\delta_{\mathrm{CH}_{2} \text { (ring) }}$ \\
\hline 108 & 1341 & 1312 & 0 & 3 & 43 & $\delta_{\mathrm{CH}_{2} \text { (chain) }}$ \\
\hline 107 & 1338 & 1310 & 3 & -9 & 106 & $\delta_{\mathrm{CH}_{2} \text { (chain) }}$ \\
\hline 106 & 1333 & 1305 & 0 & 2 & 61 & $\delta_{\mathrm{CH}_{2} \text { (chain) }}$ \\
\hline 105 & 1332 & 1304 & 5 & -15 & 112 & $\delta_{\mathrm{CH}_{2} \text { (chain) }}$ \\
\hline 104 & 1327 & 1299 & 0 & -18 & 121 & $\delta_{\mathrm{HC}-\mathrm{vinyl}}+\delta_{\mathrm{CH}_{2} \text { (chain) }}$ \\
\hline 103 & 1327 & 1299 & 1 & 29 & 22 & $\delta_{\mathrm{HC}-\mathrm{vinyl}}+\delta_{\mathrm{CH}_{2} \text { (chain) }}$ \\
\hline 102 & 1321 & 1294 & 7 & 20 & 56 & $\delta_{\mathrm{HC}-\text { vinyl }}+\delta_{\mathrm{CH}_{2} \text { (chain) }}$ \\
\hline 101 & 1317 & 1289 & 1 & -8 & 129 & $\delta_{\mathrm{CH}_{2} \text { (chain) }}$ \\
\hline 100 & 1296 & 1269 & 7 & 2 & 87 & $\delta_{\mathrm{CH}_{2} \text { (chain) }}$ \\
\hline 99 & 1293 & 1266 & 1 & 1 & 79 & $\delta_{\mathrm{CH}_{2} \text { (chain) }}$ \\
\hline 98 & 1272 & 1245 & 52 & -17 & 92 & $\delta_{\mathrm{CH}_{2}(\text { chain })}+\delta_{\mathrm{CH}_{2} \text { (ring) }}$ \\
\hline 97 & 1265 & 1238 & 142 & -25 & 92 & $\delta_{\left.\mathrm{CH}_{2} \text { (chain) }\right)}+\delta_{\mathrm{CH}_{2} \text { (ring) }}$ \\
\hline 96 & 1264 & 1237 & 1 & -1 & 93 & $\delta_{C H_{2}(\text { chain })}+\delta_{H C_{2}-\text { ring }}+\delta_{C H_{2} \text { (ring) }}$ \\
\hline 95 & 1261 & 1235 & 64 & 23 & 86 & $\delta_{\mathrm{CH}_{2}(r i n g)}+\delta_{\mathrm{HC}_{2}-\mathrm{ring}}$ \\
\hline 94 & 1246 & 1219 & 173 & -62 & 94 & $\delta_{\mathrm{CH}_{2}(\text { ring })}+\delta_{\mathrm{HC}_{2}-\text { ring }}$ \\
\hline 93 & 1240 & 1214 & 7 & -1 & 90 & $\delta_{\mathrm{CH}_{2} \text { (chain) }}$ \\
\hline 92 & 1233 & 1208 & 73 & -43 & 160 & $\delta_{\mathrm{CH}_{2}(\text { ring })}+\delta_{\mathrm{HC}_{2}-\text { ring }}$ \\
\hline 91 & 1231 & 1205 & 4 & -2 & 93 & $\delta_{\mathrm{CH}_{2} \text { (chain) }}$ \\
\hline 90 & 1216 & 1190 & 5 & -7 & 105 & $\delta_{\mathrm{CH}_{2} \text { (chain) }}$ \\
\hline 89 & 1204 & 1179 & 5 & 66 & 31 & $\delta_{\mathrm{CH}_{2} \text { (chain) }}$ \\
\hline 88 & 1190 & 1165 & 56 & 16 & 84 & $\delta_{\mathrm{CH}_{2}(\text { ring })}+\delta_{\mathrm{HC}_{2}-\text { ring }}$ \\
\hline 87 & 1159 & 1135 & 39 & -41 & 100 & $v_{C_{3}-C(\text { chain })}+\delta_{H C-\text { vinyl }}+\delta_{C_{2}(\text { ring })}$ \\
\hline 86 & 1150 & 1126 & 38 & -4 & 96 & $v_{C_{2}-N}+\delta_{C H_{2} \text { (ring) }}$ \\
\hline 85 & 1146 & 1122 & 68 & -52 & 100 & $v_{C_{2}-N}+\delta_{C H_{2}(\text { ring })}$ \\
\hline 84 & 1133 & 1109 & 14 & 13 & 76 & $v_{C_{2}-C_{3}}+\delta_{C H_{2}(\text { ring })}$ \\
\hline 83 & 1102 & 1079 & 25 & 25 & 75 & $\delta_{\mathrm{CH}_{2} \text { (chain) }}$ \\
\hline 82 & 1092 & 1069 & 16 & -16 & 106 & $\delta_{\mathrm{CH}_{2} \text { (chain) }}$ \\
\hline 81 & 1068 & 1045 & 26 & 5 & 88 & $\delta_{\mathrm{CH}_{2} \text { (chain) }}$ \\
\hline 80 & 1066 & 1044 & 10 & -7 & 97 & $\delta_{\mathrm{CH}_{2} \text { (chain) }}$ \\
\hline 79 & 1065 & 1043 & 6 & -17 & 142 & $\delta_{\mathrm{CH}_{2} \text { (chain) }}$ \\
\hline 78 & 1063 & 1041 & 8 & 8 & 85 & $\delta_{\mathrm{CH}_{2} \text { (chain) }}$ \\
\hline 77 & 1058 & 1036 & 35 & 40 & 55 & $\delta_{\mathrm{CH}_{2} \text { (chain) }}$ \\
\hline 76 & 1056 & 1034 & 96 & 5 & 68 & $\delta_{H C-v i n y l}+\delta_{C H_{2}(\text { chain })}$ \\
\hline 75 & 1054 & 1032 & 18 & -10 & 94 & $\delta_{\mathrm{HC}-\text { vinyl }}+\delta_{\mathrm{CH}_{2} \text { (chain) }}$ \\
\hline 74 & 1042 & 1020 & 1 & -2 & 102 & $\delta_{H C-v i n y l}+\delta_{C H_{2}(\text { chain })}$ \\
\hline 73 & 1040 & 1018 & 6 & -88 & 147 & $\delta_{H C-\text { vinyl }}+\delta_{C H_{2} \text { (chain) }}$ \\
\hline
\end{tabular}

*Wavenumbers scaled by 0.980 to maximize the similarity between experimental and theoretical spectra according to the statistical validation of the absolute configuration performed by CompareVOA.

**Symbols: $\delta_{\mathrm{CH}_{2} \text { (ring) }}$ : deformation of the $\mathrm{HC}-\mathrm{X}$ angles in methyl and methylene groups linked to/in the fused ring; $\delta_{\mathrm{CH}_{2} \text { (chain) }}$ : deformation of the $\mathrm{HC}-\mathrm{X}$ angles in methyl and methylene groups in the alkyl side-chain; $\delta_{\mathrm{HC}_{2}-\text { ring }}$ : deformation of the $\mathrm{HC}_{2}-\mathrm{X}$ angles; $\delta_{\mathrm{HC}-\text { vinyl }}$ : deformation of the $\mathrm{HC}-\mathrm{X}$ angles in the vinyl moiety. 
Table S3. PCM/B3LYP/cc-pVTZ calculated harmonic wavenumbers in the region of interest for the main conformer of $\mathbf{6 b}(6 b-1)$.

\begin{tabular}{|c|c|c|c|c|c|c|}
\hline $\begin{array}{c}\text { Normal } \\
\text { mode }\end{array}$ & $\begin{array}{c}\omega \\
\left(c m^{-1}\right)\end{array}$ & $\begin{array}{l}\omega_{\text {scaled }} \\
\left(\mathrm{cm}^{-1}\right)^{*}\end{array}$ & $\begin{array}{c}\text { IR } \\
\text { intensity }\end{array}$ & $\begin{array}{c}\text { Rotor } \\
\text { strength }\end{array}$ & $\begin{array}{c}\mathrm{I}-\mathrm{M} \\
\text { angle }\end{array}$ & Description** \\
\hline 122 & 1420 & 1391 & 13 & 8 & 85 & $\delta_{\mathrm{CH}_{2}(\text { ring })}$ \\
\hline 121 & 1413 & 1385 & 10 & -11 & 105 & $\delta_{\mathrm{CH}_{3} \text { (chain) }}$ \\
\hline 120 & 1413 & 1385 & 29 & 11 & 85 & $\delta_{\mathrm{CH}_{2}(\text { ring })}+\delta_{\mathrm{HC}_{2}-\mathrm{ring}}$ \\
\hline 119 & 1405 & 1377 & 28 & -12 & 93 & $\delta_{\mathrm{CH}_{2}(\mathrm{ring})}$ \\
\hline 118 & 1403 & 1375 & 3 & 1 & 88 & $\delta_{\mathrm{CH}_{2} \text { (chain) }}$ \\
\hline 117 & 1402 & 1374 & 4 & 0 & 92 & $\delta_{\mathrm{CH}_{2} \text { (chain) }}$ \\
\hline 116 & 1399 & 1371 & 3 & 1 & 89 & $\delta_{\mathrm{CH}_{2} \text { (chain) }}$ \\
\hline 115 & 1398 & 1370 & 1 & -1 & 103 & $\delta_{\mathrm{CH}_{2} \text { (chain) }}$ \\
\hline 114 & 1390 & 1362 & 1 & -4 & 111 & $\delta_{\mathrm{CH}_{2} \text { (chain) }}$ \\
\hline 113 & 1373 & 1346 & 52 & -34 & 102 & $\delta_{\left.\mathrm{CH}_{2} \text { (chain) }\right)}+\delta_{\mathrm{HC}_{2}-\text { ring }}$ \\
\hline 112 & 1372 & 1344 & 79 & -63 & 101 & $\delta_{\left.\mathrm{CH}_{2} \text { (chain) }\right)}+\delta_{\mathrm{HC}_{2}-\text { ring }}$ \\
\hline 111 & 1355 & 1328 & 12 & 28 & 75 & $\delta_{\mathrm{CH}_{2}(\text { chain })}+\delta_{\mathrm{HC}_{2}-\text { ring }}+\delta_{\mathrm{CH}_{2}(\text { ring })}$ \\
\hline 110 & 1349 & 1322 & 5 & -7 & 102 & $\delta_{\mathrm{CH}_{2}(\text { chain })}+\delta_{\mathrm{HC}_{2}-\text { ring }}+\delta_{\mathrm{CH}_{2} \text { (ring) }}$ \\
\hline 109 & 1344 & 1317 & 7 & -2 & 94 & $\delta_{\mathrm{CH}_{2}(\text { chain })}+\delta_{\mathrm{HC}_{2}-\text { ring }}+\delta_{\mathrm{CH}_{2} \text { (ring) }}$ \\
\hline 108 & 1341 & 1314 & 0 & 0 & 76 & $\delta_{\mathrm{CH}_{2} \text { (chain) }}$ \\
\hline 107 & 1339 & 1312 & 1 & 1 & 87 & $\delta_{\mathrm{CH}_{2} \text { (chain) }}$ \\
\hline 106 & 1334 & 1307 & 0 & 1 & 82 & $\delta_{\mathrm{CH}_{2} \text { (chain) }}$ \\
\hline 105 & 1332 & 1305 & 3 & 1 & 85 & $\delta_{\mathrm{CH}_{2} \text { (chain) }}$ \\
\hline 104 & 1327 & 1301 & 1 & 33 & 53 & $\delta_{\mathrm{HC}-\text { vinyl }}+\delta_{\mathrm{CH}_{2} \text { (chain) }}$ \\
\hline 103 & 1327 & 1300 & 6 & -53 & 155 & $\delta_{\mathrm{HC}-\mathrm{vinyl}}+\delta_{\mathrm{CH}_{2} \text { (chain) }}$ \\
\hline 102 & 1321 & 1295 & 4 & -9 & 121 & $\delta_{\mathrm{HC}-\text { vinyl }}+\delta_{\mathrm{CH}_{2} \text { (chain) }}$ \\
\hline 101 & 1317 & 1291 & 3 & -7 & 127 & $\delta_{\mathrm{CH}_{2} \text { (chain) }}$ \\
\hline 100 & 1297 & 1272 & 8 & 3 & 86 & $\delta_{\mathrm{CH}_{2} \text { (chain) }}$ \\
\hline 99 & 1292 & 1267 & 9 & -6 & 102 & $\delta_{\mathrm{CH}_{2} \text { (chain) }}$ \\
\hline 98 & 1280 & 1254 & 159 & -40 & 92 & $v_{N-C}+\delta_{H C_{2}-r i n g}$ \\
\hline 97 & 1270 & 1245 & 3 & 7 & 82 & $\delta_{\mathrm{CH}_{2} \text { (chain) }}$ \\
\hline 96 & 1265 & 1240 & 163 & -24 & 93 & $\delta_{\left.\mathrm{CH}_{2} \text { (chain) }\right)}+\delta_{\mathrm{CH}_{2}(\text { ring })}$ \\
\hline 95 & 1263 & 1238 & 34 & 9 & 86 & $\delta_{\mathrm{CH}_{2} \text { (chain) }}$ \\
\hline 94 & 1255 & 1229 & 103 & -87 & 107 & $\delta_{\mathrm{CH}_{2}(\text { ring })}+\delta_{\mathrm{HC}_{2}-\text { ring }}$ \\
\hline 93 & 1243 & 1218 & 17 & -22 & 107 & $\delta_{\mathrm{CH}_{2} \text { (chain) }}$ \\
\hline 92 & 1233 & 1209 & 16 & -19 & 98 & $\delta_{\mathrm{CH}_{2}(\text { ring })}+\delta_{\mathrm{HC}_{2}-\text { ring }}$ \\
\hline 91 & 1231 & 1207 & 1 & 0 & 78 & $\delta_{\mathrm{CH}_{2} \text { (chain) }}$ \\
\hline 90 & 1220 & 1195 & 2 & -5 & 103 & $\delta_{\mathrm{CH}_{2} \text { (chain) }}$ \\
\hline 89 & 1207 & 1183 & 2 & -8 & 99 & $\delta_{\mathrm{CH}_{2} \text { (chain) }}$ \\
\hline 88 & 1191 & 1167 & 70 & 80 & 79 & $\delta_{\mathrm{CH}_{2}(\text { ring })}+\delta_{\mathrm{HC}_{2}-\text { ring }}$ \\
\hline 87 & 1169 & 1145 & 20 & 16 & 84 & $v_{N-C}+\delta_{H C_{2}-\text { ring }}$ \\
\hline 86 & 1157 & 1134 & 11 & 2 & 87 & $v_{C_{2}-C_{3}}+\delta_{H C-v i n y l}$ \\
\hline 85 & 1141 & 1118 & 23 & 37 & 55 & $\delta_{H C-v i n y l}+\delta_{C_{2}(\text { chain })}$ \\
\hline 84 & 1129 & 1106 & 69 & 7 & 87 & $v_{C_{2}-C_{3}}+\delta_{H C-v i n y l}$ \\
\hline 83 & 1108 & 1086 & 11 & 30 & 59 & $\delta_{\mathrm{CH}_{2} \text { (chain) }}$ \\
\hline 82 & 1090 & 1068 & 30 & 14 & 84 & $\delta_{\mathrm{CH}_{2} \text { (chain) }}$ \\
\hline 81 & 1073 & 1051 & 25 & -44 & 106 & $\delta_{\mathrm{CH}_{2} \text { (chain) }}$ \\
\hline 80 & 1067 & 1045 & 1 & 5 & 54 & $\delta_{\mathrm{CH}_{2} \text { (chain) }}$ \\
\hline 79 & 1065 & 1044 & 16 & -34 & 114 & $\delta_{\mathrm{CH}_{2} \text { (chain) }}$ \\
\hline 78 & 1064 & 1043 & 48 & 96 & 69 & $\delta_{\mathrm{CH}_{2} \text { (chain) }}$ \\
\hline 77 & 1062 & 1040 & 105 & -4 & 90 & $\delta_{\mathrm{CH}_{2} \text { (chain) }}$ \\
\hline 76 & 1057 & 1036 & 1 & -8 & 126 & $\delta_{H C-\text { vinyl }}+\delta_{\left.C H_{2} \text { (chain }\right)}$ \\
\hline 75 & 1051 & 1030 & 10 & 7 & 69 & $\delta_{H C-\text { vinyl }}+\delta_{\mathrm{CH}_{2}(\text { chain })}$ \\
\hline 74 & 1047 & 1026 & 23 & -75 & 108 & $\delta_{\mathrm{HC}-\mathrm{vinyl}}+\delta_{\mathrm{CH}_{2}(\text { chain })}+v_{\mathrm{CO}}$ \\
\hline 73 & 1041 & 1020 & 11 & 1 & 89 & $\delta_{H C-\text { vinyl }}+\delta_{\mathrm{CH}_{2}(\text { chain })}$ \\
\hline
\end{tabular}

*Wavenumbers scaled by 0.980 to maximize the similarity between experimental and theoretical spectra according to the statistical validation of the absolute configuration performed by CompareVOA.

**Symbols: $\delta_{\mathrm{CH}_{2}(\text { ring })}$ : deformation of the $\mathrm{HC}-\mathrm{X}$ angles in methyl and methylene groups linked to/in the fused ring; $\delta_{\mathrm{CH}_{2} \text { (chain) }}$ : deformation of the $\mathrm{HC}-\mathrm{X}$ angles in methyl and methylene groups in the alkyl side-chain; $\delta_{\mathrm{HC}_{2}-\text { ring }}$ : deformation of the $\mathrm{HC}_{2}-\mathrm{X}$ angles; $\delta_{H C-v i n y l}$ : deformation of the $\mathrm{HC}-\mathrm{X}$ angles in the vinyl moiety. 


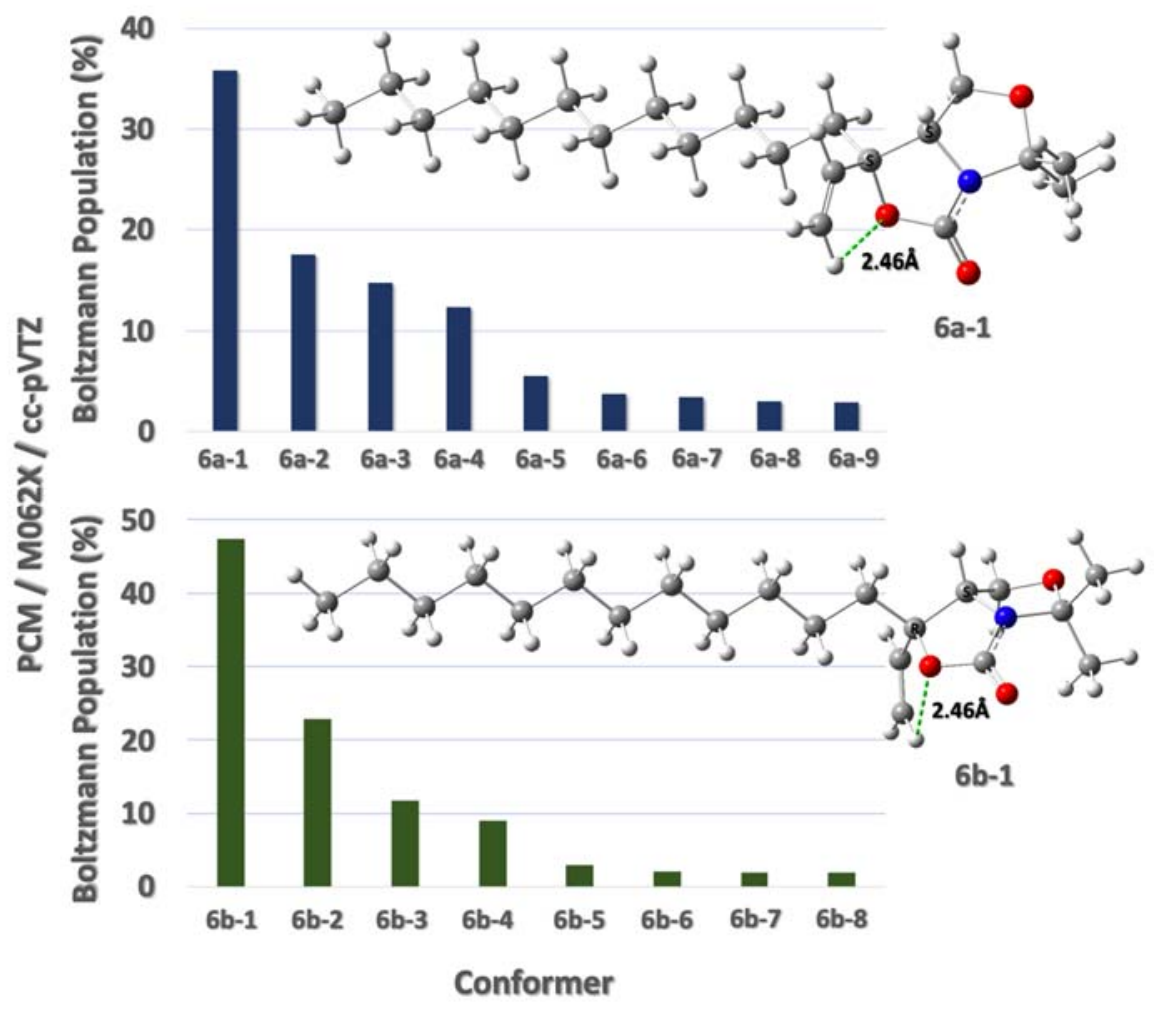

Figure S2. PCM/M062X/cc-pVTZ Boltzmann populations of the main conformers of 6 a and $\mathbf{6 b}$ and molecular structure of the global minima.

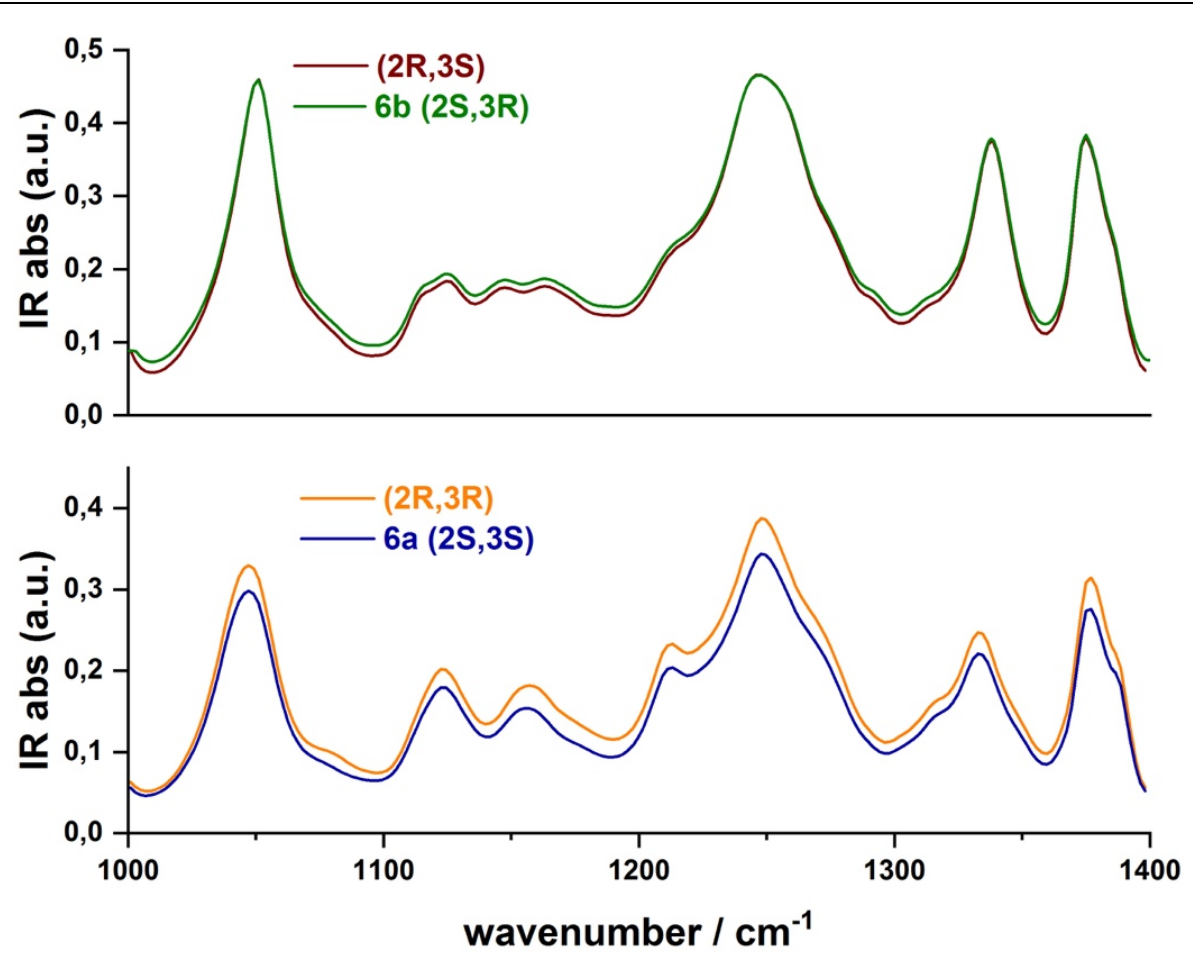

Figure S3. IR spectra of $\mathbf{6 a}$ and $\mathbf{6 b}$ and their respective enantiomers 


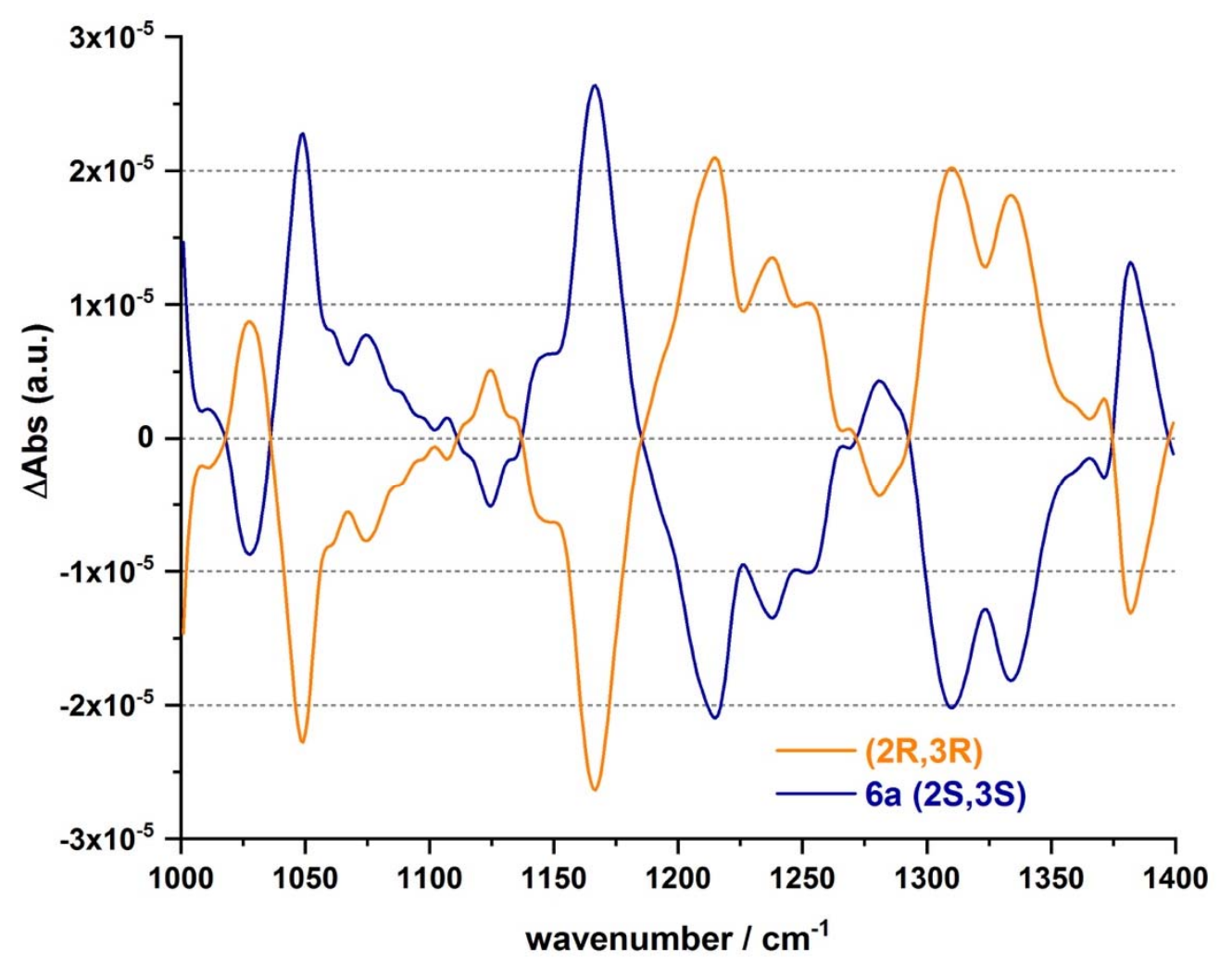

Figure S4. VCD spectra of $\mathbf{6 a}$ and its enantiomer.

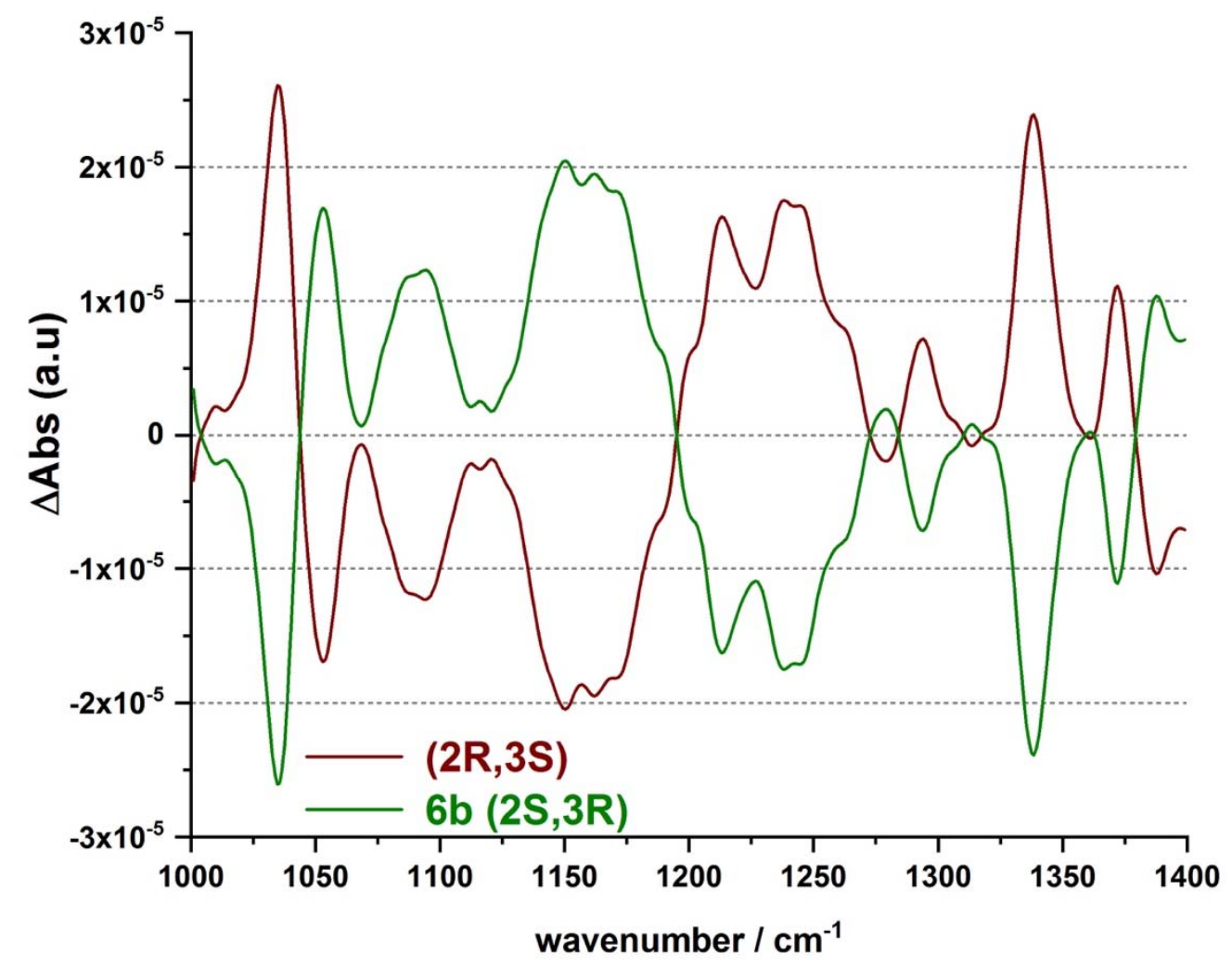

Figure S5. VCD spectra of $\mathbf{6 b}$ and its enantiomer. 


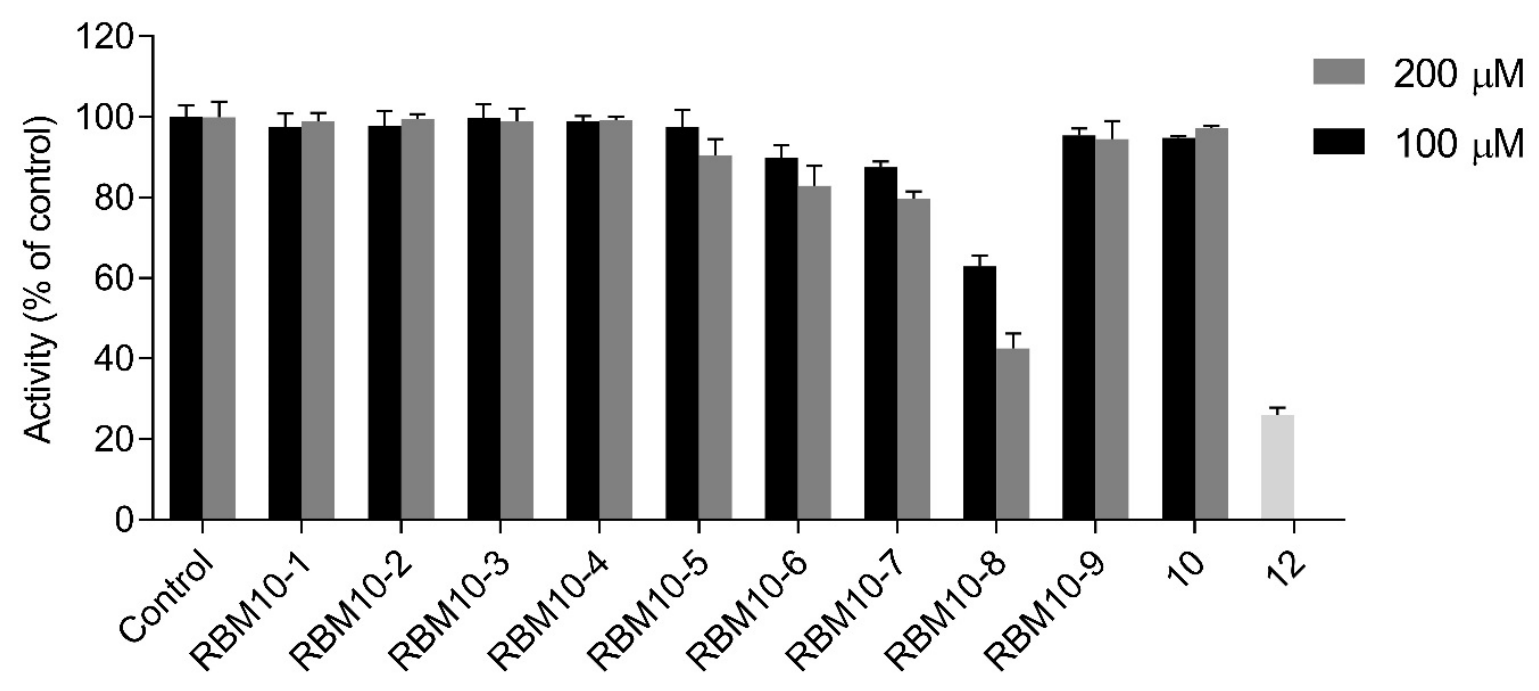

Figure S6. hS1PL activities of compounds RBM10 (100 and $200 \mu \mathrm{M})$ and vinyl ketone 10 using RBM148 as fluorogenic substrate. Inhibitor $\mathbf{1 2}^{1}(200 \mathrm{nM})$ was used as reference. Enzyme activity is expressed as \% relative to control. Data (mean $\pm \mathrm{SD}$ ) were obtained from two experiments with triplicates. 


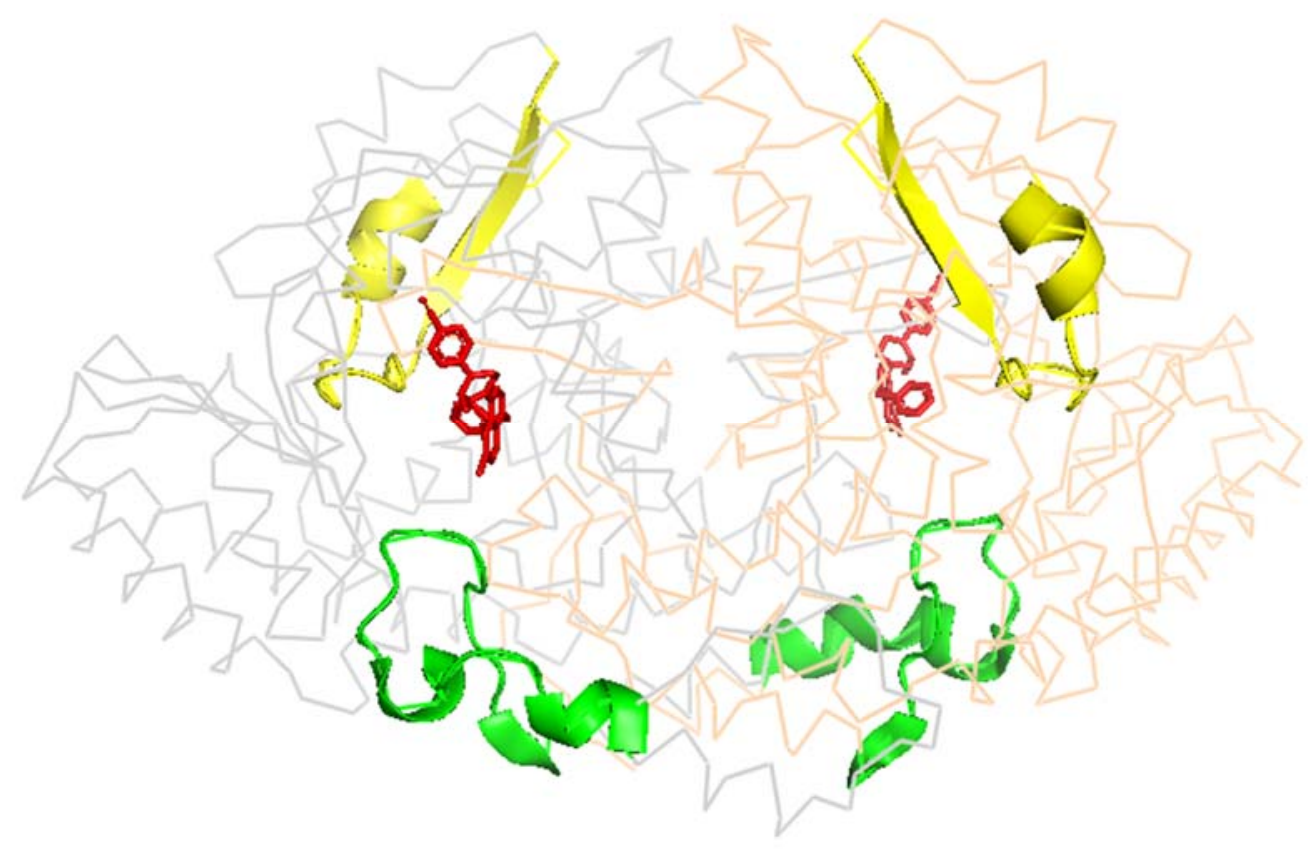

Figure S7. Backbone of hSPL dimer (PDB 4Q6R, chain A in gray and chain B in salmon) showing inhibitor 13 (red) and highlighted fragments 132-155 (green) and 279-303 (yellow).

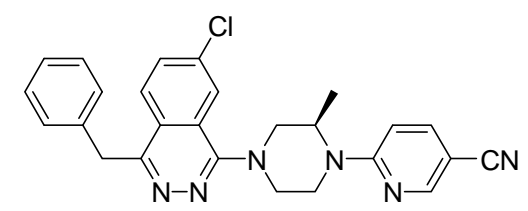

13 


\section{Cartesian Coordinates matrices of the optimized (PCM/M062X/cc-pVTZ) structures of the set of conformers of $6 a$ and $6 b$.}

\section{6a-1}

C $\quad 4.60746900-1.60752200-1.00370500$

$\mathrm{H} \quad 3.98991000-2.06324100-0.22212100$

$\mathrm{H} \quad 4.34031700-2.02600900-1.97102000$

C $\quad 6.49302900 \quad-0.89311800 \quad 0.17244400$

$\begin{array}{llll}\mathrm{C} & 4.54894400 & -0.08695900 & -0.93204800\end{array}$

$\mathrm{H} \quad 4.94643100 \quad 0.34412100-1.85127300$

$\begin{array}{llll}\mathrm{C} & 7.77720900 & -0.32922900 & -0.40158400\end{array}$

$\begin{array}{llll}\mathrm{H} & 8.48507600 & -1.13789100 & -0.57745100\end{array}$

$\begin{array}{llll}\mathrm{H} & 8.21323900 & 0.38369700 & 0.29640200\end{array}$

$\begin{array}{llll}\mathrm{H} & 7.57430700 & 0.17414800 & -1.34550900\end{array}$

$\begin{array}{llll}\text { C } & 6.71566000 & -1.57953800 & 1.50604300\end{array}$

$\mathrm{H} \quad 7.11042400 \quad-0.89105700 \quad 2.24742300$

$\mathrm{H} \quad 7.42796100 \quad-2.38587300 \quad 1.34019500$

$\mathrm{H} \quad 5.78656600 \quad-2.00226100 \quad 1.88529200$

O $\quad 5.98212500 \quad-1.85388800 \quad-0.77099200$

$\mathrm{N} \quad \begin{array}{llll}\mathrm{N} & 5.44284700 & 0.14843000 & 0.19523300\end{array}$

$\begin{array}{llll}\mathrm{C} & 4.69324000 & 0.46056300 & 1.31174600\end{array}$

$\begin{array}{llll}\mathrm{C} & 3.26770800 & 0.63902300 & -0.48530000\end{array}$

$\begin{array}{llll}\mathrm{O} & 5.05739100 & 0.55721300 & 2.44875100\end{array}$

$\begin{array}{llll}\mathrm{O} & 3.40711500 & 0.68970200 & 0.94193600\end{array}$

C $\quad 3.27036700 \quad 2.03934900 \quad-1.04409700$

$\mathrm{H} \quad 3.13424100 \quad 2.08947800 \quad-2.12038900$

C $\quad 3.43131300 \quad 3.14180700 \quad-0.33349100$

$\mathrm{H} \quad 3.43508600 \quad 4.11289600 \quad-0.80831200$

H $\quad 3.55810000 \quad 3.10661400 \quad 0.74044800$

C $\quad 1.98248800 \quad-0.10060100 \quad-0.83949800$

$\mathrm{H} \quad \begin{array}{llll}1.95733600 & -0.22772900 & -1.92607800\end{array}$

H $\quad 2.02500300 \quad-1.09876300 \quad-0.40036200$

$\begin{array}{llll}0.71732500 & 0.60531200 & -0.36710800\end{array}$

$\begin{array}{llll}0.80027800 & 0.80827800 & 0.70325700\end{array}$

$\begin{array}{llll}0.62920600 & 1.57556000 & -0.86117700\end{array}$

$-0.53486800-0.21913800-0.63781000$

$-0.59949500-0.44320500-1.70748600$

$\begin{array}{llll}-0.45243500 & -1.18312500 & -0.12644800\end{array}$

$\begin{array}{llll}-1.81317900 & 0.47882400 & -0.19171700\end{array}$

$\begin{array}{llll}-1.89988500 & 1.43958600 & -0.70849300\end{array}$

$\begin{array}{lll}-1.74400100 & 0.71015700 & 0.87552200\end{array}$

$\begin{array}{llll}-3.06707800 & -0.34702800 & -0.44883500\end{array}$

$\begin{array}{llll}-3.13296600 & -0.58265500 & -1.51576200\end{array}$

$\begin{array}{lll}-2.98117400 & -1.30593600 & 0.07179000\end{array}$

$\begin{array}{llll}-4.34688900 & 0.35180200 & -0.00807700\end{array}$

$\begin{array}{llll}-4.27980900 & 0.58985300 & 1.05805900\end{array}$

$\begin{array}{llll}-4.43413600 & 1.30966500 & -0.53046800\end{array}$

$\begin{array}{llll}-5.60063000 & -0.47564100 & -0.26119200\end{array}$

$\begin{array}{llll}-5.51379700 & -1.43262800 & 0.26290000\end{array}$

$\begin{array}{llll}-5.66653500 & -0.71554100 & -1.32714100\end{array}$

$\begin{array}{lll}-6.88106800 & 0.22359100 & 0.17713900\end{array}$

$\begin{array}{llll}-6.81461900 & 0.46469300 & 1.24269200\end{array}$

$\begin{array}{llll}-6.96864700 & 1.18001100 & -0.34787800\end{array}$

$\begin{array}{llll}-8.13455000 & -0.60489800 & -0.07404400\end{array}$

$\begin{array}{lll}-8.04711500 & -1.56093900 & 0.45173600\end{array}$

$-8.20052200-0.84680400-1.13954100$

$\begin{array}{lll}-9.41521100 & 0.09419500 & 0.36315100\end{array}$

$\begin{array}{lll}-9.35009200 & 0.33674700 & 1.42863500\end{array}$

$\begin{array}{llll}-9.50400700 & 1.05020000 & -0.16273900\end{array}$

$\begin{array}{llll}-10.66916600 & -0.73443800 & 0.11291400\end{array}$

$\begin{array}{llll}-10.57870300 & -1.68839900 & 0.63899400\end{array}$

$\begin{array}{llll}-10.73238100 & -0.97575500 & -0.95140800\end{array}$

$\begin{array}{llll}-11.94069700 & -0.02159900 & 0.55518800\end{array}$

$\begin{array}{llll}-12.82754200 & -0.62679900 & 0.36950400\end{array}$ 
$\begin{array}{llll}\mathrm{H} & -12.06080400 & 0.92236000 & 0.02131900\end{array}$

$\begin{array}{llll}\mathrm{H} & -11.90629100 & 0.20476500 & 1.62198000\end{array}$

6a-2

$\begin{array}{llll}\text { C } & -3.93926400 & -0.31380800 & -1.86127400\end{array}$

$\mathrm{H} \quad-4.05529300 \quad 0.76972900 \quad-1.75012500$

$\mathrm{H} \quad-3.49162100 \quad-0.53651800 \quad-2.82675500$

C $\quad-5.55546900 \quad-1.15628900 \quad-0.40395200$

C $\quad-3.20565300 \quad-0.91175300 \quad-0.66767900$

$\mathrm{H} \quad-2.87509900 \quad-1.92361800 \quad-0.90348700$

C $\quad-5.98931200 \quad-2.59892400 \quad-0.23966300$

$\mathrm{H} \quad-6.82336800 \quad-2.80829100 \quad-0.90784700$

$\mathrm{H} \quad-6.30006000 \quad-2.77727900 \quad 0.78853500$

$\mathrm{H} \quad-\quad-5.16464300 \quad-3.26657700 \quad-0.48404700$

$\begin{array}{llll}\mathrm{C} & -6.66059500 & -0.18201600 & -0.04496600\end{array}$

$\mathrm{H} \quad-7.50192400 \quad-0.38918700 \quad-0.70396600$

$\begin{array}{lllll}\mathrm{H} & -6.33709600 & 0.84640000 & -0.19872100\end{array}$

$\mathrm{H} \quad-6.97084800 \quad-0.29552000 \quad 0.98958500$

$\begin{array}{llll}\mathrm{O} & -5.19277400 & -0.96760300 & -1.78474800\end{array}$

$\begin{array}{llll}\mathrm{N} & -4.28352800 & -0.91975300 & 0.31391300\end{array}$

$\begin{array}{llll}\text { C } & -4.08788900 & 0.11081700 & 1.21151400\end{array}$

$\begin{array}{llll}\text { C } & -2.10628500 & -0.12388900 & 0.06645300\end{array}$

$\begin{array}{llll}\mathrm{O} & -4.83882900 & 0.51851200 & 2.05094200\end{array}$

$\begin{array}{lllll}\mathrm{O} & -2.84463700 & 0.62888400 & 1.03956100\end{array}$

C $\quad-1.18713900 \quad-1.09218400 \quad 0.76750500$

$\begin{array}{lllll}\mathrm{H} & -0.56650300 & -1.68119500 & 0.09866600\end{array}$

$\begin{array}{llll}\mathrm{C} & -1.11304300 & -1.25905700 & 2.07636600\end{array}$

$\mathrm{H} \quad-0.43801400 \quad-1.98566700 \quad 2.50631200$

$\begin{array}{llll}\mathrm{H} & -1.71915500 & -0.67264600 & 2.75414500\end{array}$

$\begin{array}{llll}\mathrm{C} & -1.32971100 & 0.83517000 & -0.82901400\end{array}$

$\mathrm{H} \quad-2.03798100 \quad 1.51087500 \quad-1.31116200$

$\mathrm{H} \quad-0.85705500 \quad 0.24586800 \quad-1.62106600$

$\begin{array}{lllll}\mathrm{C} & -0.27656900 & 1.65024300 & -0.08850800\end{array}$

$\begin{array}{llll}\mathrm{H} & 0.45358000 & 0.97469000 & 0.36153700\end{array}$

$\mathrm{H} \quad-0.75249000 \quad 2.18723500 \quad 0.73627100$

$\begin{array}{lllll}\mathrm{C} & 0.42660000 & 2.63980800 & -1.01042800\end{array}$

$\mathrm{H} \quad-0.32383300 \quad 3.25569500 \quad-1.51265500$

$\begin{array}{lllll}\mathrm{H} & 0.94580200 & 2.08969800 & -1.80044600\end{array}$

$\begin{array}{llll}\mathrm{C} & 1.40858400 & 3.55842100 & -0.28378000\end{array}$

$\begin{array}{llll}\mathrm{H} & 0.84813600 & 4.20603600 & 0.39516700\end{array}$

$\begin{array}{llll}\mathrm{H} & 1.88446500 & 4.21466000 & -1.01703800\end{array}$

$\begin{array}{llll}\mathrm{C} & 2.49501700 & 2.83109000 & 0.50807400\end{array}$

$\begin{array}{llll}\mathrm{H} & 3.16027800 & 3.57505700 & 0.95371400\end{array}$

$\begin{array}{llll}\mathrm{H} & 2.04693400 & 2.28871000 & 1.34524300\end{array}$

$\begin{array}{lllll}\mathrm{C} & 3.32613100 & 1.86388300 & -0.32705200\end{array}$

$\mathrm{H} \quad 3.71436200 \quad 2.38586300 \quad-1.20822200$

$\begin{array}{llll}\mathrm{H} & 2.69108500 & 1.05686200 & -0.70527400\end{array}$

$\begin{array}{lllll}\mathrm{C} & 4.48463400 & 1.25361500 & 0.45195900\end{array}$

$\begin{array}{llll}\mathrm{H} & 5.13554900 & 2.05359600 & 0.81800300\end{array}$

$\begin{array}{llll}\mathrm{H} & 4.09390400 & 0.74708700 & 1.34029400\end{array}$

$\begin{array}{llll}\mathrm{C} & 5.30873000 & 0.26901400 & -0.36760600\end{array}$

$\mathrm{H} \quad 4.65782200 \quad-0.53213800 \quad-0.73201600$

$\begin{array}{lllll}5.69769100 & 0.77527700 & -1.25672200\end{array}$

$\begin{array}{llll}\mathrm{C} & 6.46727500 & -0.34039300 & 0.41166900\end{array}$

$\begin{array}{llll}\mathrm{H} & 6.07812200 & -0.84623500 & 1.30092000\end{array}$

$\begin{array}{llll}\mathrm{H} & 7.11863600 & 0.46023200 & 0.77592200\end{array}$

$\begin{array}{llll}\mathrm{C} & 7.29098400 & -1.32543700 & -0.40750200\end{array}$

$\mathrm{H} \quad 6.64005800 \quad-2.12593800 \quad-0.77357600$

$\begin{array}{llll}\mathrm{H} & 7.68244400 & -0.81975500 & -1.29599500\end{array}$

C $\quad 8.44824600 \quad-1.93727900 \quad 0.37233500$

$\mathrm{H} \quad 9.09687800 \quad-1.13665300 \quad 0.73729600$

$\begin{array}{llll}\mathrm{H} & 8.05530000 & -2.44192000 & 1.25884000\end{array}$

C $\quad 9.26208200 \quad-2.91941900 \quad-0.46043500$

$\mathrm{H} \quad 8.63542000 \quad-3.74003000 \quad-0.81317400$

$\mathrm{H} \quad 10.08451500 \quad-3.34789300 \quad 0.11158300$

$\mathrm{H} \quad 9.68450300 \quad-2.42626900 \quad-1.33727200$ 
6a-3

$\mathrm{C}$

$\begin{array}{llll}4.69842400 & 1.54475300 & -0.46361400\end{array}$

$\begin{array}{llll}4.06669200 & 1.41859200 & -1.34958500\end{array}$

$\begin{array}{llll}4.68409200 & 2.58563000 & -0.14977900\end{array}$

$\begin{array}{llll}6.18036900 & -0.25185900 & -0.62122300\end{array}$

$\begin{array}{llll}4.32165700 & 0.54807700 & 0.62505400\end{array}$

$\begin{array}{lll}4.76981900 & 0.84606600 & 1.57324900\end{array}$

$\begin{array}{llll}7.39903600 & -0.51760500 & 0.23948900\end{array}$

$\begin{array}{llll}8.27573800 & -0.06051700 & -0.21702200\end{array}$

$\begin{array}{llll}7.56059800 & -1.59045800 & 0.33154700\end{array}$

$\begin{array}{llll}7.25484400 & -0.09212400 & 1.23133500\end{array}$

$6.31647700 \quad-0.85231300-2.00694200$

$6.43427200-1.93112000-1.96388700$

$\begin{array}{llll}7.19600400 & -0.40652800 & -2.46822000\end{array}$

$\begin{array}{llll}5.44391800 & -0.62307500 & -2.61677800\end{array}$

$\begin{array}{llll}6.03729200 & 1.17635600 & -0.74009100\end{array}$

$\begin{array}{llll}4.95095000 & -0.65552800 & 0.09535600\end{array}$

$3.98011100 \quad-1.47533700 \quad-0.44435600$

$\begin{array}{llll}2.86409600 & 0.09297100 & 0.81588300\end{array}$

$4.12458000-2.46184500-1.10816400$

$\begin{array}{llll}2.75306100 & -1.01740300 & -0.08630400\end{array}$

$\begin{array}{llll}2.67521900 & -0.38600700 & 2.23312100\end{array}$

$\begin{array}{lll}2.70830900 & 0.40313000 & 2.97848800\end{array}$

$\begin{array}{llll}2.49445700 & -1.64460900 & 2.59308500\end{array}$

$\begin{array}{llll}2.38027000 & -1.91345300 & 3.63383700\end{array}$

$\begin{array}{llll}2.44996500 & -2.43956500 & 1.86046800\end{array}$

$\begin{array}{llll}1.83196100 & 1.15079300 & 0.44160500\end{array}$

$\begin{array}{llll}2.00243600 & 1.44962100 & -0.59407900\end{array}$

$\begin{array}{llll}2.01292400 & 2.03197400 & 1.06486200\end{array}$

$\begin{array}{llll}0.38996100 & 0.68475500 & 0.60177000\end{array}$

$\begin{array}{llll}0.25409200 & -0.24986200 & 0.05247600\end{array}$

$\begin{array}{llll}0.18885700 & 0.45958900 & 1.65164300\end{array}$

$\begin{array}{lll}-0.60792100 & 1.72275700 & 0.10422700\end{array}$

$\begin{array}{llll}-0.44756800 & 2.66678400 & 0.63486800\end{array}$

$\begin{array}{llll}-0.41974500 & 1.92948600 & -0.95391900\end{array}$

$\begin{array}{llll}-2.05668000 & 1.28551000 & 0.28033100\end{array}$

$\begin{array}{llll}-2.25429300 & 1.10350600 & 1.34196300\end{array}$

$\begin{array}{llll}-2.20113600 & 0.32912300 & -0.22825900\end{array}$

$\begin{array}{llll}-3.05141100 & 2.31326300 & -0.24849800\end{array}$

$\begin{array}{llll}-2.81639400 & 3.28760000 & 0.18861500\end{array}$

$\begin{array}{llll}-2.91474400 & 2.41973900 & -1.32926900\end{array}$

$\begin{array}{llll}-4.51061400 & 1.97354300 & 0.04419700\end{array}$

$\begin{array}{llll}-4.65834600 & 1.92042500 & 1.12751900\end{array}$

$\begin{array}{llll}-5.14574600 & 2.78882400 & -0.31269400\end{array}$

$\begin{array}{llll}-4.98576500 & 0.66978700 & -0.58813000\end{array}$

$-4.43459100 \quad-0.17366600-0.16394600$

$\begin{array}{llll}-4.75439400 & 0.68157300 & -1.65852400\end{array}$

$\begin{array}{llll}-6.47835500 & 0.42977300 & -0.39570300\end{array}$

$\begin{array}{lll}-6.70545200 & 0.45928900 & 0.67325500\end{array}$

$\begin{array}{llll}-7.04022300 & 1.25142200 & -0.85258100\end{array}$

$\begin{array}{lll}-6.94757700 & -0.89326500 & -0.99148900\end{array}$

$\begin{array}{llll}-6.45369800 & -1.71720700 & -0.46674100\end{array}$

$\begin{array}{lll}-6.61378500 & -0.94788500 & -2.03122900\end{array}$

$\begin{array}{llll}-8.45937800 & -1.09807600 & -0.94087200\end{array}$

$\begin{array}{llll}-8.95202200 & -0.30078500 & -1.50683900\end{array}$

$\begin{array}{llll}-8.71161000 & -2.03399300 & -1.44694700\end{array}$

$\begin{array}{llll}-9.03716800 & -1.13536400 & 0.47029900\end{array}$

$\begin{array}{lll}-8.88006100 & -0.17234400 & 0.95990000\end{array}$

$\begin{array}{llll}-8.48995800 & -1.87570700 & 1.06105800\end{array}$

$\begin{array}{llll}-10.52417100 & -1.46734300 & 0.47550800\end{array}$

$\begin{array}{llll}-11.08592700 & -0.73071500 & -0.10120800\end{array}$

$\begin{array}{llll}-10.93072300 & -1.48156900 & 1.48635900\end{array}$

$\begin{array}{llll}-10.70488700 & -2.44519000 & 0.02659500\end{array}$

6a-4

C $\quad 2.64417800 \quad-0.82729300 \quad-1.83716900$

$\mathrm{H} \quad 1.57545900 \quad-0.73945400 \quad-1.61284300$ 


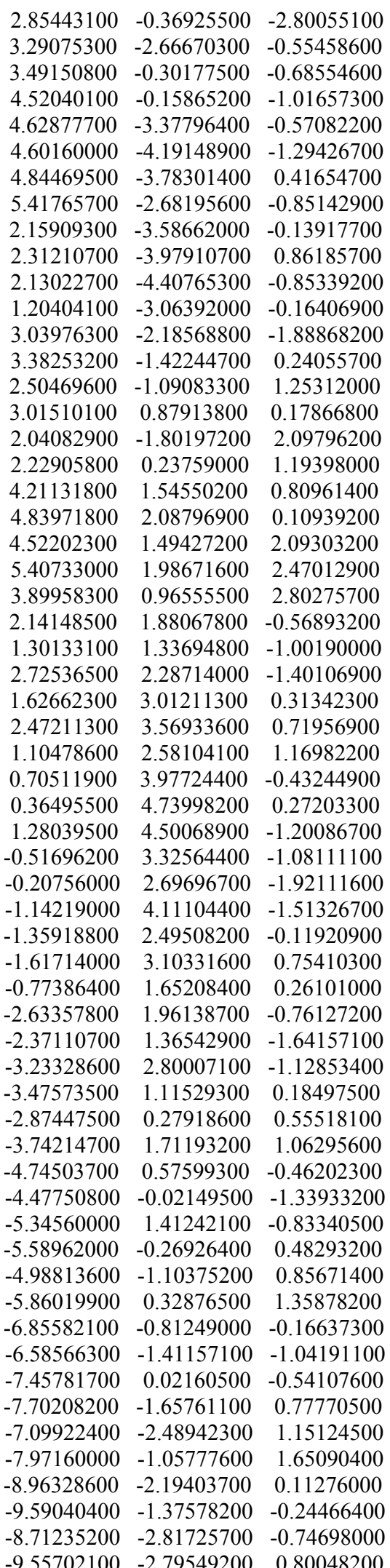

$\begin{array}{llll}-9.55702100 & -2.79549200 & 0.80048200\end{array}$

\section{6a-5}

$\begin{array}{lrrr}\mathrm{C} & -4.48120800 & 1.11520800 & -1.18797800 \\ \mathrm{H} & -4.33504600 & 1.81788500 & -0.36044700 \\ \mathrm{H} & -4.26941700 & 1.61232200 & -2.13148100 \\ \mathrm{C} & -5.96138900 & -0.45210500 & -0.29125600 \\ \mathrm{C} & -3.69864500 & -0.17151500 & -0.95851000\end{array}$


$\mathrm{H} \quad-3.64304100 \quad-0.74397100 \quad-1.88478100$

C $\quad \begin{array}{lllll} & -6.65223900 & -1.58372300 & -1.02552900\end{array}$

$\mathrm{H} \quad-\quad-7.61481400 \quad-1.24089100 \quad-1.40210200$

$\mathrm{H} \quad-6.80973800 \quad-2.42248000-0.34932700$

$\begin{array}{lllll}\mathrm{H} & & -6.04137200 & -1.91207000 & -1.86494900\end{array}$

$\begin{array}{llll}\mathrm{C} & -6.75880800 & 0.02132300 & 0.90828200\end{array}$

$\begin{array}{llll}\mathrm{H} & -6.90077800 & -0.77617400 & 1.63183600\end{array}$

$\begin{array}{llll}\mathrm{H} & -7.72668600 & 0.35805300 & 0.54096600\end{array}$

$\mathrm{H} \quad-6.26037200 \quad 0.85357200 \quad 1.40278300$

$\begin{array}{lllll}\mathrm{O} & -5.81465300 & 0.64015600 & -1.21894500\end{array}$

$\begin{array}{llll}\mathrm{N} & -4.56345600 & -0.82272700 & 0.01785600\end{array}$

$\begin{array}{llll}\mathrm{C} & -3.99641800 & -0.71004100 & 1.27175600\end{array}$

$\begin{array}{llll}\mathrm{C} & -2.34343800 & -0.15720900 & -0.22981000\end{array}$

$\begin{array}{llll}\mathrm{O} & -4.48298700 & -0.97138900 & 2.33455400\end{array}$

$\begin{array}{llll}\mathrm{O} & -2.71995300 & -0.26318600 & 1.15078100\end{array}$

C $\quad \begin{array}{llll}\text { C } & -1.55127600 & -1.37529000 & -0.63291700\end{array}$

$\mathrm{H} \quad-1.19165200 \quad-1.35418700 \quad-1.65740500$

$\begin{array}{llll}\mathrm{C} & -1.29912400 & -2.41145500 & 0.14761200\end{array}$

$\mathrm{H} \quad-0.73496900 \quad-3.25782600 \quad-0.21828900$

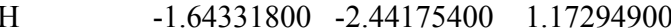

$\begin{array}{lllll}\mathrm{C} & & -1.54326100 & 1.12387200 & -0.43655200\end{array}$

$\begin{array}{llll}\mathrm{H} & -1.36332500 & 1.23757500 & -1.51027600\end{array}$

$\begin{array}{llll}\mathrm{H} & -2.15600200 & 1.97038500 & -0.12232600\end{array}$

$\begin{array}{llll}\text { C } & -0.21986900 & 1.14770300 & 0.31856600\end{array}$

$\begin{array}{llll}\mathrm{H} & -0.40856300 & 0.96319600 & 1.37953300\end{array}$

$\begin{array}{llll}0.41049900 & 0.32758600 & -0.03111700\end{array}$

$\begin{array}{lll}0.50945400 & 2.47517000 & 0.14789600\end{array}$

$\begin{array}{lll}0.74206700 & 2.62510100 & -0.91008600\end{array}$

$\begin{array}{lll}-0.16207100 & 3.29074700 & 0.42819700\end{array}$

$\begin{array}{lll}1.78399100 & 2.58178000 & 0.98524800\end{array}$

$\begin{array}{lll}1.50790700 & 2.59976100 & 2.04258000\end{array}$

$\begin{array}{llll}2.26430300 & 3.54075800 & 0.77419200\end{array}$

$\begin{array}{llll}2.79874100 & 1.45980200 & 0.76088200\end{array}$

$\begin{array}{llll}3.66777600 & 1.65628500 & 1.39118000\end{array}$

$\begin{array}{lll}2.38445500 & 0.50715000 & 1.10436200\end{array}$

$\begin{array}{lll}3.24259400 & 1.31529000 & -0.69118900\end{array}$

$\begin{array}{lll}2.36952200 & 1.11939500 & -1.31868700\end{array}$

$\begin{array}{llll}3.66107200 & 2.26725400 & -1.03541800\end{array}$

$\begin{array}{lll}4.26307600 & 0.20158700 & -0.91705300\end{array}$

$\begin{array}{llll}4.45270000 & 0.10489300 & -1.98954300\end{array}$

$\begin{array}{lll}3.83237000 & -0.75187700 & -0.59441700\end{array}$

$\begin{array}{llll}5.59497400 & 0.41389400 & -0.20508300\end{array}$

$\begin{array}{llll}5.98196400 & 1.40816400 & -0.45234000\end{array}$

$\begin{array}{lll}5.44839600 & 0.40097900 & 0.87775700\end{array}$

$\begin{array}{lll}6.63411900 & -0.63792700 & -0.57372000\end{array}$

$\begin{array}{lll}6.80426600 & -0.61492500 & -1.65472500\end{array}$

$\begin{array}{llll}6.23627500 & -1.63250100 & -0.34802100\end{array}$

$\begin{array}{lll}7.96193200 & -0.45187400 & 0.14880200\end{array}$

$\begin{array}{llll}8.35701100 & 0.54546100 & -0.06963300\end{array}$

$\begin{array}{lll}7.79406200 & -0.48380200 & 1.23001400\end{array}$

$\begin{array}{lll}9.00378900 & -1.49786900 & -0.22821400\end{array}$

$\begin{array}{llll}9.17239900 & -1.46231900 & -1.30770300\end{array}$

$\begin{array}{llll}8.60622800 & -2.49304100 & -0.01236000\end{array}$

$\begin{array}{lll}10.32415600 & -1.30115800 & 0.50554900\end{array}$

$\begin{array}{lll}11.05813300 & -2.05564300 & 0.22399100\end{array}$

$10.17935100-1.36052200 \quad 1.58533800$

$10.74844400 \quad-0.32088000 \quad 0.28295400$

6a-6

$\begin{array}{llll}\mathrm{C} & 1.38628500 & -1.33854600 & -1.45135000 \\ \mathrm{H} & 0.54623400 & -0.99320100 & -0.83850100 \\ \mathrm{H} & 1.15209000 & -1.20009800 & -2.50400200 \\ \mathrm{C} & 2.45418000 & -2.86044100 & -0.04190300 \\ \mathrm{C} & 2.69377500 & -0.69846000 & -1.00220000 \\ \mathrm{H} & 3.45994900 & -0.84642100 & -1.76370900 \\ \mathrm{C} & 3.59437300 & -3.79852400 & -0.38264200 \\ \mathrm{H} & 3.19394700 & -4.75329700 & -0.72035700\end{array}$




\begin{tabular}{|c|c|c|c|}
\hline & 4.21529800 & -3.96100600 & 0.49693600 \\
\hline $\mathrm{H}$ & 4.20486800 & -3.37085400 & -1.17630300 \\
\hline & 1.58952100 & -3.39581700 & 1.08293800 \\
\hline & 2.16070600 & -3.52519700 & 1.99749300 \\
\hline & 1.19470900 & -4.35710300 & 0.75884000 \\
\hline & 0.75702800 & -2.72374700 & 1.28664700 \\
\hline & 1.64697200 & -2.71161100 & -1.22493400 \\
\hline & 2.96815200 & -1.49224400 & 0.18922500 \\
\hline & 2.67899800 & -0.74272200 & 1.31168200 \\
\hline & 2.72796000 & 0.73784300 & -0.44989500 \\
\hline U & 2.62442200 & -1.10313300 & 2.45269400 \\
\hline $\mathrm{O}$ & 2.47587100 & 0.55043500 & 0.95085100 \\
\hline $\mathrm{C}$ & 4.10812000 & 1.31240400 & -0.64308000 \\
\hline $\mathrm{H}$ & 4.36084800 & 1.52661100 & -1.67727900 \\
\hline $\mathrm{C}$ & 4.97834000 & 1.54298500 & 0.32435100 \\
\hline & 5.95799200 & 1.94442400 & 0.10616500 \\
\hline & 4.73579400 & 1.34323600 & 1.35968900 \\
\hline & 1.65100200 & 1.64718100 & -1.03116300 \\
\hline & 1.77764100 & 1.67553700 & -2.11822400 \\
\hline & 0.68315900 & 1.18546200 & -0.83294100 \\
\hline & 1.67816000 & 3.06086900 & -0.46120800 \\
\hline & 1.65200300 & 3.00170000 & 0.62855600 \\
\hline & 2.62487500 & 3.53827600 & -0.71831300 \\
\hline & 0.52564900 & 3.92913800 & -0.96693900 \\
\hline & 0.60507300 & 4.91233000 & -0.49701200 \\
\hline & 0.64092900 & 4.08892400 & -2.04236400 \\
\hline & -0.86702100 & 3.35985500 & -0.69058000 \\
\hline & -1.03232000 & 2.47384400 & -1.30759400 \\
\hline & -1.61573300 & 4.09006200 & -1.01013700 \\
\hline & -1.10132300 & 3.02029600 & 0.77864000 \\
\hline $\mathrm{H}$ & -0.43543900 & 2.20669400 & 1.08343100 \\
\hline $\mathrm{H}$ & -0.82283900 & 3.88553200 & 1.38731400 \\
\hline U & -2.53983200 & 2.61959100 & 1.09404800 \\
\hline $\mathrm{H}$ & -2.64285200 & 2.48059600 & 2.17330200 \\
\hline $\mathrm{H}$ & -3.21457100 & 3.43737300 & 0.82183400 \\
\hline $\mathrm{C}$ & -2.99209500 & 1.34272000 & 0.39275900 \\
\hline & -2.26540400 & 0.54706900 & 0.59236400 \\
\hline & -2.99826400 & 1.49175900 & -0.69031600 \\
\hline $\mathrm{C}$ & -4.37552400 & 0.88333400 & 0.83584800 \\
\hline & -4.37306400 & 0.72418700 & 1.91849900 \\
\hline & -5.09855700 & 1.68316800 & 0.64601500 \\
\hline & -4.84098500 & -0.39010600 & 0.14069700 \\
\hline & -4.12621100 & -1.19520800 & 0.34239400 \\
\hline & -4.82751100 & -0.22956100 & -0.94054700 \\
\hline & -6.23176500 & -0.83040800 & 0.58442400 \\
\hline & -6.24997300 & -0.88818600 & 1.67603200 \\
\hline & -6.95714800 & -0.05971100 & 0.30433600 \\
\hline & -6.67474700 & -2.17204500 & 0.00511200 \\
\hline & -7.64500100 & -2.43802700 & 0.42901700 \\
\hline & -5.97468300 & -2.94832500 & 0.32557400 \\
\hline & -6.77475300 & -2.17395700 & -1.51665800 \\
\hline & -7.18301200 & -3.11505400 & -1.88397600 \\
\hline & -5.79939400 & -2.03429500 & -1.98230900 \\
\hline & -7.42626300 & -1.36845400 & -1.86129500 \\
\hline
\end{tabular}

\section{6a-7}

$\begin{array}{lrrr}\mathrm{C} & -3.57286100 & -1.73150500 & -0.00531000 \\ \mathrm{H} & -3.15296400 & -1.60489300 & -1.00905100 \\ \mathrm{H} & -3.05462400 & -2.53713500 & 0.50902900 \\ \mathrm{C} & -5.72108300 & -0.84468400 & -0.22359400 \\ \mathrm{C} & -3.59705400 & -0.40878100 & 0.74983200 \\ \mathrm{H} & -3.76866100 & -0.58970900 & 1.81122300 \\ \mathrm{C} & -6.84215800 & -0.88643600 & 0.79526700 \\ \mathrm{H} & -7.44971500 & -1.77580700 & 0.63452200 \\ \mathrm{H} & -7.46796300 & -0.00122700 & 0.69343600 \\ \mathrm{H} & -6.42961600 & -0.91767500 & 1.80244700 \\ \mathrm{C} & -6.24266000 & -0.76818200 & -1.64534200\end{array}$




\begin{tabular}{|c|c|c|c|}
\hline & -6.83535200 & 0.12794300 & -1.80382500 \\
\hline & -6.85982000 & -1.64927600 & -1.81222200 \\
\hline$\pi$ & -5.42185400 & -0.77171600 & -2.36098200 \\
\hline $\mathrm{O}$ & -4.95057900 & -2.05166400 & -0.06966600 \\
\hline$N$ & -4.75403200 & 0.21935500 & 0.12416000 \\
\hline $\mathrm{C}$ & -4.32255100 & 1.18633400 & -0.76158500 \\
\hline $\mathrm{C}$ & -2.49946200 & 0.64835000 & 0.53535100 \\
\hline $\mathrm{O}$ & -4.96447600 & 1.79358400 & -1.57028900 \\
\hline $\mathrm{O}$ & -2.99548500 & 1.40634200 & -0.57777500 \\
\hline $\mathrm{C}$ & -2.41649200 & 1.53393200 & 1.75287400 \\
\hline $\mathrm{H}$ & -2.01271600 & 1.04386100 & 2.63391100 \\
\hline $\mathrm{C}$ & -2.79735700 & 2.79844300 & 1.80105100 \\
\hline $\mathrm{H}$ & -2.71901200 & 3.36736300 & 2.71683800 \\
\hline $\mathrm{H}$ & -3.19224500 & 3.30165000 & 0.92842700 \\
\hline $\mathrm{C}$ & -1.13713300 & 0.06275500 & 0.18063900 \\
\hline $\mathrm{H}$ & -0.84512400 & -0.60809800 & 0.99294400 \\
\hline $\mathrm{H}$ & -1.24417500 & -0.54719300 & -0.71883400 \\
\hline $\mathrm{C}$ & -0.06627700 & 1.12444800 & -0.05350100 \\
\hline $\mathrm{H}$ & -0.47039900 & 1.88308400 & -0.72608900 \\
\hline $\mathrm{H}$ & 0.15885100 & 1.63035700 & 0.88833100 \\
\hline $\mathrm{C}$ & 1.21934000 & 0.55119700 & -0.64304700 \\
\hline $\mathrm{H}$ & 1.00201500 & 0.09679400 & -1.61464200 \\
\hline $\mathrm{H}$ & 1.91427000 & 1.37188800 & -0.83670700 \\
\hline $\mathrm{C}$ & 1.90737500 & -0.47677400 & 0.24866100 \\
\hline $\mathrm{H}$ & 1.26244200 & -1.35010000 & 0.38086200 \\
\hline $\mathrm{H}$ & 2.05522400 & -0.04844300 & 1.24603900 \\
\hline $\mathrm{C}$ & 3.25014900 & -0.93722000 & -0.30539400 \\
\hline $\mathrm{H}$ & 3.10338900 & -1.36708200 & -1.30204500 \\
\hline $\mathrm{H}$ & 3.89504000 & -0.06397700 & -0.44119200 \\
\hline $\mathrm{C}$ & 3.94137500 & -1.95941200 & 0.58979100 \\
\hline $\mathrm{H}$ & 4.15771200 & -1.49876800 & 1.55777800 \\
\hline $\mathrm{H}$ & 3.24711200 & -2.77901300 & 0.79344400 \\
\hline $\mathrm{C}$ & 5.22308000 & -2.53901800 & -0.00866700 \\
\hline $\mathrm{H}$ & 4.96558100 & -3.12044300 & -0.89794300 \\
\hline $\mathrm{H}$ & 5.66133400 & -3.24106600 & 0.70566300 \\
\hline $\mathrm{C}$ & 6.27393300 & -1.49476500 & -0.38474900 \\
\hline $\mathrm{H}$ & 7.15873400 & -2.00682600 & -0.77393500 \\
\hline $\mathrm{H}$ & 5.89723900 & -0.87706100 & -1.20284600 \\
\hline $\mathrm{C}$ & 6.69209600 & -0.60728600 & 0.78427900 \\
\hline $\mathrm{H}$ & 6.92601900 & -1.24324700 & 1.64329500 \\
\hline $\mathrm{H}$ & 5.85034100 & 0.02225300 & 1.08889600 \\
\hline $\mathrm{C}$ & 7.88954900 & 0.28794800 & 0.47722500 \\
\hline $\mathrm{H}$ & 8.16573900 & 0.84109800 & 1.37913600 \\
\hline $\mathrm{H}$ & 8.75297500 & -0.33449900 & 0.22122900 \\
\hline $\mathrm{C}$ & 7.64259500 & 1.28479600 & -0.65081900 \\
\hline $\mathrm{H}$ & 6.72666300 & 1.84447200 & -0.43834700 \\
\hline $\mathrm{H}$ & 7.46683900 & 0.74896100 & -1.58560500 \\
\hline 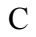 & 8.80705100 & 2.25014900 & -0.83330100 \\
\hline n & 8.62604800 & 2.95072500 & -1.64792800 \\
\hline $\boldsymbol{H}$ & 9.72685200 & 1.70714400 & -1.05653300 \\
\hline & 8.97764400 & 2.82834700 & 0.076052 \\
\hline
\end{tabular}

\section{6a-8}

$\begin{array}{lrrr}\mathrm{C} & -4.33025000 & -0.91052700 & -1.37318000 \\ \mathrm{H} & -3.56565400 & -0.40360400 & -1.97179500 \\ \mathrm{H} & -4.40426800 & -1.95103700 & -1.67985000 \\ \mathrm{C} & -5.69969000 & 0.84915000 & -0.68337700 \\ \mathrm{C} & -4.08746300 & -0.70350300 & 0.11647300 \\ \mathrm{H} & -4.68684800 & -1.40930900 & 0.69203300 \\ \mathrm{C} & -7.02615200 & 0.75516700 & 0.04340500 \\ \mathrm{H} & -7.83851100 & 0.72004100 & -0.68099900 \\ \mathrm{H} & -7.15750700 & 1.62307900 & 0.68766700 \\ \mathrm{H} & -7.05538200 & -0.14782000 & 0.65114200 \\ \mathrm{C} & -5.59461800 & 2.10848900 & -1.52146800 \\ \mathrm{H} & -5.67416800 & 3.00186700 & -0.90905600 \\ \mathrm{H} & -6.40898500 & 2.08623900 & -2.24351000 \\ \mathrm{H} & -4.64777100 & 2.14000300 & -2.05850100\end{array}$




\begin{tabular}{|c|c|c|c|}
\hline & -5.59575700 & -0.29959700 & -1.54565200 \\
\hline$N$ & -4.58179700 & 0.65978800 & 0.26667100 \\
\hline $\mathrm{C}$ & -3.50928000 & 1.52244500 & 0.37354100 \\
\hline $\mathrm{C}$ & -2.66064000 & -0.59369900 & 0.68243700 \\
\hline $\mathrm{O}$ & -3.51237100 & 2.71993500 & 0.34610100 \\
\hline $\mathrm{O}$ & -2.37039000 & 0.80578100 & 0.55483100 \\
\hline $\mathrm{C}$ & -2.67502900 & -0.97622000 & 2.14084300 \\
\hline $\mathrm{H}$ & -2.85276000 & -2.03202100 & 2.32274000 \\
\hline $\mathrm{C}$ & -2.50435600 & -0.13876800 & 3.14875200 \\
\hline $\mathrm{H}$ & -2.54213700 & -0.48612300 & 4.17168400 \\
\hline $\mathrm{H}$ & -2.31710600 & 0.91365800 & 2.98142000 \\
\hline $\mathrm{C}$ & -1.62314900 & -1.39594500 & -0.09511000 \\
\hline $\mathrm{H}$ & -1.64428600 & -1.07180400 & -1.13699300 \\
\hline $\mathrm{H}$ & -1.92956300 & -2.44638200 & -0.07839600 \\
\hline $\mathrm{C}$ & -0.20620400 & -1.25693500 & 0.44824900 \\
\hline $\mathrm{H}$ & -0.16078900 & -1.64994100 & 1.46649700 \\
\hline $\mathrm{H}$ & 0.04878500 & -0.19652200 & 0.51312700 \\
\hline $\mathrm{C}$ & 0.81589300 & -1.97826000 & -0.42113700 \\
\hline $\mathrm{H}$ & 0.54190500 & -3.03471100 & -0.50629900 \\
\hline $\mathrm{H}$ & 0.78266900 & -1.56964800 & -1.43598100 \\
\hline $\mathrm{C}$ & 2.23668300 & -1.86755300 & 0.11727000 \\
\hline $\mathrm{H}$ & 2.27913200 & -2.29627100 & 1.12408800 \\
\hline $\mathrm{H}$ & 2.49239800 & -0.80945600 & 0.22429400 \\
\hline $\mathrm{C}$ & 3.26124500 & -2.56181600 & -0.77243800 \\
\hline $\mathrm{H}$ & 3.27356700 & -2.07490500 & -1.75125900 \\
\hline $\mathrm{H}$ & 2.93612800 & -3.59011700 & -0.95184900 \\
\hline $\mathrm{C}$ & 4.67162900 & -2.59008000 & -0.18330400 \\
\hline $\mathrm{H}$ & 5.34473300 & -3.06846900 & -0.89977500 \\
\hline $\mathrm{H}$ & 4.66736800 & -3.22399100 & 0.70743500 \\
\hline $\mathrm{C}$ & 5.24103100 & -1.22094400 & 0.19097900 \\
\hline $\mathrm{H}$ & 4.65830900 & -0.78540200 & 1.00829400 \\
\hline $\mathrm{H}$ & 6.24971700 & -1.36639000 & 0.58186400 \\
\hline $\mathrm{C}$ & 5.28029300 & -0.23455600 & -0.97179400 \\
\hline $\mathrm{H}$ & 4.26765500 & -0.08671500 & -1.35456300 \\
\hline $\mathrm{H}$ & 5.85923400 & -0.67047500 & -1.79321900 \\
\hline $\mathrm{C}$ & 5.86643600 & 1.12767500 & -0.60628700 \\
\hline $\mathrm{H}$ & 5.76230400 & 1.80081600 & -1.46153400 \\
\hline $\mathrm{H}$ & 5.27592500 & 1.56807000 & 0.20361400 \\
\hline $\mathrm{C}$ & 7.33367900 & 1.09127800 & -0.19243600 \\
\hline $\mathrm{H}$ & 7.91356300 & 0.57035200 & -0.96200900 \\
\hline $\mathrm{H}$ & 7.45192300 & 0.51170100 & 0.72667400 \\
\hline $\mathrm{C}$ & 7.92077900 & 2.48086000 & 0.02516900 \\
\hline $\mathrm{H}$ & 7.82295700 & 3.05966600 & -0.89690600 \\
\hline $\mathrm{H}$ & 7.32879600 & 3.00471000 & 0.78036100 \\
\hline $\mathrm{C}$ & 9.38173600 & 2.43950900 & 0.45446000 \\
\hline $\mathrm{H}$ & 9.49502400 & 1.89024400 & 1.39038900 \\
\hline 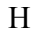 & 9.99219100 & 1.93838700 & -0.29840800 \\
\hline & 9.78671900 & 3.44019800 & 0.60235300 \\
\hline
\end{tabular}

\section{6a-9}

$\begin{array}{lrrr}\mathrm{C} & 4.92345200 & 0.07552100 & -1.81539600 \\ \mathrm{H} & 3.96046200 & -0.34471800 & -2.11025800 \\ \mathrm{H} & 5.27708800 & 0.74684000 & -2.59255800 \\ \mathrm{C} & 5.72565100 & -1.54782300 & -0.36602800 \\ \mathrm{C} & 4.87712100 & 0.71734200 & -0.42900800 \\ \mathrm{H} & 5.65756700 & 1.47688100 & -0.34802600 \\ \mathrm{C} & 7.08635600 & -1.96968200 & 0.14292200 \\ \mathrm{H} & 7.50688600 & -2.73052400 & -0.51292900 \\ \mathrm{H} & 6.98688000 & -2.37834500 & 1.14757600 \\ \mathrm{H} & 7.75124600 & -1.10886700 & 0.16839200 \\ \mathrm{C} & 4.74855100 & -2.71116200 & -0.43125300 \\ \mathrm{H} & 4.56360700 & -3.10691200 & 0.56515200 \\ \mathrm{H} & 5.17140600 & -3.49429800 & -1.05789500 \\ \mathrm{H} & 3.79941800 & -2.39103500 & -0.86162200 \\ \mathrm{O} & 5.89249700 & -0.95167800 & -1.64955000 \\ \mathrm{~N} & 5.18052100 & -0.42182800 & 0.41233400 \\ \mathrm{C} & 4.37655600 & -0.47744600 & 1.50778000\end{array}$




$\begin{array}{lrrr}\mathrm{C} & 3.55594500 & 1.24398900 & 0.20309300 \\ \mathrm{O} & 4.34123800 & -1.31992000 & 2.36335600 \\ \mathrm{O} & 3.59236100 & 0.63525900 & 1.52346900 \\ \mathrm{C} & 3.63637100 & 2.73232000 & 0.38602300 \\ \mathrm{H} & 4.32077000 & 3.04048500 & 1.16961200 \\ \mathrm{C} & 3.01438400 & 3.63807200 & -0.34956300 \\ \mathrm{H} & 3.17735400 & 4.69354300 & -0.18277200 \\ \mathrm{H} & 2.32349800 & 3.36766000 & -1.13787400 \\ \mathrm{C} & 2.29671000 & 0.74282100 & -0.49479900 \\ \mathrm{H} & 2.39245000 & -0.34058000 & -0.61438200 \\ \mathrm{H} & 2.25501800 & 1.16277600 & -1.50363000 \\ \mathrm{C} & 0.99977800 & 1.03046800 & 0.25448000 \\ \mathrm{H} & 0.86512500 & 2.10650800 & 0.37580300 \\ \mathrm{H} & 1.06949700 & 0.60977700 & 1.25909400 \\ \mathrm{C} & -0.21004400 & 0.44500900 & -0.46391200 \\ \mathrm{H} & -0.27047900 & 0.85957200 & -1.47556500 \\ \mathrm{H} & -0.07561700 & -0.63473100 & -0.58210000 \\ \mathrm{C} & -1.52016400 & 0.71031100 & 0.26649300 \\ \mathrm{H} & -1.65636100 & 1.78973000 & 0.38486500 \\ \mathrm{H} & -1.45934500 & 0.29640700 & 1.27747200 \\ \mathrm{C} & -2.73162900 & 0.12434500 & -0.44727300 \\ \mathrm{H} & -2.59478800 & -0.95526400 & -0.56419400 \\ \mathrm{H} & -2.78989000 & 0.53706400 & -1.45947200 \\ \mathrm{C} & -4.04383400 & 0.38893700 & 0.27983400 \\ \mathrm{H} & -4.18086600 & 1.46847600 & 0.39745500 \\ \mathrm{H} & -3.98606500 & -0.02419100 & 1.29162900 \\ \mathrm{C} & -5.25450600 & -0.19728800 & -0.43518400 \\ \mathrm{H} & -5.31103900 & 0.21513500 & -1.44755500 \\ \mathrm{H} & -5.11738300 & -1.27692100 & -0.55194100 \\ \mathrm{C} & -6.56794800 & 0.06739100 & 0.28967600 \\ \mathrm{H} & -6.70510400 & 1.14699000 & 0.40678300 \\ \mathrm{H} & -6.51189300 & -0.34535700 & 1.30180600 \\ \mathrm{C} & -7.77796600 & -0.51879300 & -0.42655500 \\ \mathrm{H} & -7.83308500 & -0.10676400 & -1.43915900 \\ \mathrm{H} & -7.64104200 & -1.59853900 & -0.54276600 \\ \mathrm{C} & -9.09206800 & -0.25345100 & 0.29644100 \\ \mathrm{H} & -9.23011000 & 0.82626500 & 0.41286200 \\ \mathrm{H} & -9.03824300 & -0.66539800 & 1.30921500 \\ \mathrm{C} & -10.30236900 & -0.83965700 & -0.41995900 \\ \mathrm{H} & -10.35379500 & -0.42787500 & -1.43129900 \\ \mathrm{H} & -10.16311400 & -1.91785600 & -0.53431700 \\ \mathrm{C} & -11.60829100 & -0.56458800 & 0.31451700 \\ \mathrm{H} & -11.58474200 & -0.99073100 & 1.31866700 \\ \mathrm{H} & -11.77680000 & 0.50867800 & 0.41543500 \\ & -12.46326100 & -0.99017000 & -0.21001900 \\ & & & \end{array}$

\section{6b-1}

C

$\begin{array}{rrr}5.41477600 & -1.67137800 & 0.75379500 \\ 5.42639500 & -1.18572000 & 1.73178800 \\ 5.26076400 & -2.73978800 & 0.87679500 \\ 6.66644500 & -0.17338200 & -0.48138200 \\ 4.41266800 & -1.00815000 & -0.19124600 \\ 4.08995900 & -1.71664300 & -0.95755600 \\ 7.48719700 & -0.20944600 & -1.75115200 \\ 8.52059300 & -0.46037300 & -1.51640300 \\ 7.45652900 & 0.76819300 & -2.23019700 \\ 7.08037400 & -0.95618200 & -2.42980500 \\ 7.19562800 & 0.83984200 & 0.52219500 \\ 7.17049200 & 1.84171200 & 0.09942700 \\ 8.22223700 & 0.58059400 & 0.77485800 \\ 6.59127100 & 0.83079600 & 1.42996800 \\ 6.65119200 & -1.48112800 & 0.08058400 \\ 5.23303600 & 0.04041200 & -0.76724700 \\ 4.58468500 & 1.23790000 & -0.78815200 \\ 3.18971800 & -0.20586200 & 0.33651000 \\ 5.00805000 & 2.30720000 & -1.13453100 \\ 3.30395800 & 1.04656600 & -0.37137100\end{array}$




$\begin{array}{lrrr}\mathrm{C} & 3.27017000 & 0.05609600 & 1.81564000 \\ \mathrm{H} & 3.08342600 & -0.81243700 & 2.44069500 \\ \mathrm{C} & 3.54679100 & 1.22761100 & 2.36464900 \\ \mathrm{H} & 3.59231900 & 1.34038700 & 3.43898200 \\ \mathrm{H} & 3.72190200 & 2.10687500 & 1.75850000 \\ \mathrm{C} & 1.87331400 & -0.86563900 & -0.05084000 \\ \mathrm{H} & 1.85162200 & -1.86119900 & 0.40404500 \\ \mathrm{H} & 1.88278700 & -1.01067000 & -1.13385100 \\ \mathrm{C} & 0.63657300 & -0.07534800 & 0.35418500 \\ \mathrm{H} & 0.60238600 & 0.02632900 & 1.44172400 \\ \mathrm{H} & 0.71221300 & 0.93697500 & -0.04948900 \\ \mathrm{C} & -0.65013100 & -0.72897200 & -0.13415600 \\ \mathrm{H} & -0.71372100 & -1.74789500 & 0.26125400 \\ \mathrm{H} & -0.61861200 & -0.82382200 & -1.22385600 \\ \mathrm{C} & -1.89853800 & 0.04583900 & 0.26752600 \\ \mathrm{H} & -1.92658500 & 0.14675400 & 1.35692600 \\ \mathrm{H} & -1.83603400 & 1.06234200 & -0.13260100 \\ \mathrm{C} & -3.18905000 & -0.60683500 & -0.21097700 \\ \mathrm{H} & -3.25159700 & -1.62262600 & 0.19209800 \\ \mathrm{H} & -3.15989600 & -0.71082400 & -1.30012600 \\ \mathrm{C} & -4.43742400 & 0.17013400 & 0.18712700 \\ \mathrm{H} & -4.46256200 & 0.28051800 & 1.27581500 \\ \mathrm{H} & -4.37791400 & 1.18361000 & -0.22168700 \\ \mathrm{C} & -5.72932900 & -0.48700400 & -0.28162600 \\ \mathrm{H} & -5.70364800 & -0.59980400 & -1.37003900 \\ \mathrm{H} & -5.78939700 & -1.49966700 & 0.12954400 \\ \mathrm{C} & -6.97746400 & 0.29149700 & 0.11426400 \\ \mathrm{H} & -6.91966300 & 1.30260000 & -0.30079200 \\ \mathrm{H} & -7.00045300 & 0.40848800 & 1.20232100 \\ \mathrm{C} & -8.27000200 & -0.36915700 & -0.34787300 \\ \mathrm{H} & -8.24680200 & -0.48745900 & -1.43579200 \\ \mathrm{H} & -8.32801900 & -1.37982000 & 0.06849300 \\ \mathrm{C} & -9.51800600 & 0.40973900 & 0.04685400 \\ \mathrm{H} & -9.46240100 & 1.41977700 & -0.37154600 \\ \mathrm{H} & -9.54075300 & 0.53067500 & 1.13463500 \\ \mathrm{C} & -10.81138800 & -0.25272200 & -0.41137100 \\ \mathrm{H} & -10.86513800 & -1.26085700 & 0.00789600 \\ \mathrm{H} & -10.78681900 & -0.37301900 & -1.49761000 \\ \mathrm{C} & -12.04952100 & 0.53840900 & -0.00929600 \\ \mathrm{H} & -12.96525000 & 0.05135400 & -0.34316500 \\ \mathrm{H} & -12.10372600 & 0.64763300 & 1.07497000 \\ \mathrm{H} & -12.02577800 & 1.54018700 & -0.44098800\end{array}$

\section{6b-2}

$\begin{array}{lrrr}\mathrm{C} & 2.72004700 & -2.07328600 & -0.50862200 \\ \mathrm{H} & 2.53420900 & -2.28012400 & 0.54747000 \\ \mathrm{H} & 1.92350500 & -2.49998800 & -1.11179000 \\ \mathrm{C} & 5.01637900 & -1.81883000 & -0.49524400 \\ \mathrm{C} & 2.95151100 & -0.58269700 & -0.75623800 \\ \mathrm{H} & 2.70693400 & -0.32896400 & -1.79027100 \\ \mathrm{C} & 6.13883400 & -1.91917700 & -1.50387100 \\ \mathrm{H} & 6.52633300 & -2.93669200 & -1.52617900 \\ \mathrm{H} & 6.94011300 & -1.23748500 & -1.22214000 \\ \mathrm{H} & 5.76966400 & -1.65242900 & -2.49194300 \\ \mathrm{C} & 5.46333700 & -2.19958200 & 0.90791200 \\ \mathrm{H} & 6.25616800 & -1.53678200 & 1.24734300 \\ \mathrm{H} & 5.83127500 & -3.22396300 & 0.89276000 \\ \mathrm{H} & 4.62948900 & -2.13022200 & 1.60728700 \\ \mathrm{O} & 3.94707500 & -2.65281000 & -0.92881000 \\ \mathrm{~N} & 4.37979200 & -0.48613800 & -0.52250300 \\ \mathrm{C} & 4.69346700 & 0.57447900 & 0.27220700 \\ \mathrm{C} & 2.39382700 & 0.50685100 & 0.20259100 \\ \mathrm{O} & 5.76943400 & 0.88800600 & 0.70408900 \\ \mathrm{O} & 3.56375600 & 1.29750200 & 0.49914000 \\ \mathrm{C} & 1.85442700 & -0.07418600 & 1.48112400 \\ \mathrm{H} & 0.90737800 & -0.59646800 & 1.37675300 \\ \mathrm{C} & 2.44457800 & 0.01125100 & 2.66208100\end{array}$




$\begin{array}{lrrr}\mathrm{H} & 1.99790100 & -0.43538700 & 3.53955800 \\ \mathrm{H} & 3.38068000 & 0.53978200 & 2.78728300 \\ \mathrm{C} & 1.36617900 & 1.39288700 & -0.48793900 \\ \mathrm{H} & 0.54080200 & 0.74876600 & -0.80702800 \\ \mathrm{H} & 1.82621400 & 1.79335300 & -1.39539200 \\ \mathrm{C} & 0.85469900 & 2.53375000 & 0.38271600 \\ \mathrm{H} & 0.31212800 & 2.12649100 & 1.23819800 \\ \mathrm{H} & 1.71064300 & 3.07296000 & 0.79158700 \\ \mathrm{C} & -0.03755400 & 3.50816600 & -0.38520000 \\ \mathrm{H} & 0.56261000 & 4.01806100 & -1.14301000 \\ \mathrm{H} & -0.38567600 & 4.27979100 & 0.30556100 \\ \mathrm{C} & -1.24847600 & 2.86288400 & -1.05891300 \\ \mathrm{H} & -0.91964100 & 2.20021100 & -1.86493600 \\ \mathrm{H} & -1.84068000 & 3.64575100 & -1.53928200 \\ \mathrm{C} & -2.14506800 & 2.08186000 & -0.10454000 \\ \mathrm{H} & -1.59590700 & 1.23269700 & 0.31491500 \\ \mathrm{H} & -2.41316700 & 2.71917500 & 0.74459800 \\ \mathrm{C} & -3.41343100 & 1.56707500 & -0.77348700 \\ \mathrm{H} & -3.98096300 & 2.41411000 & -1.17089400 \\ \mathrm{H} & -3.14162600 & 0.95047400 & -1.63641000 \\ \mathrm{C} & -4.30377200 & 0.75883900 & 0.16165300 \\ \mathrm{H} & -3.73857900 & -0.09276200 & 0.55369300 \\ \mathrm{H} & -4.57075600 & 1.37300400 & 1.02733400 \\ \mathrm{C} & -5.57495200 & 0.25315000 & -0.50848300 \\ \mathrm{H} & -5.30764800 & -0.35662800 & -1.37731300 \\ \mathrm{H} & -6.14230600 & 1.10505800 & -0.89603500 \\ \mathrm{C} & -6.46362900 & -0.56113000 & 0.42311600 \\ \mathrm{H} & -5.89672700 & -1.41396500 & 0.80974900 \\ \mathrm{H} & -6.73042200 & 0.04812700 & 1.29238300 \\ \mathrm{C} & -7.73500800 & -1.06479900 & -0.24765100 \\ \mathrm{H} & -7.46907100 & -1.67281700 & -1.11827900 \\ \mathrm{H} & -8.30361500 & -0.21237400 & -0.63279400 \\ \mathrm{C} & -8.62368500 & -1.88171900 & 0.68216100 \\ \mathrm{H} & -8.05442400 & -2.73266700 & 1.06534700 \\ \mathrm{H} & -8.88802900 & -1.27313600 & 1.55088300 \\ \mathrm{C} & -9.89044300 & -2.37706000 & -0.00373400 \\ \mathrm{H} & -9.64576400 & -3.00776800 & -0.85978700 \\ \mathrm{H} & -10.51439700 & -2.95920500 & 0.67372700 \\ \mathrm{H} & -10.48492200 & -1.53894600 & -0.37061900 \\ & & & \\ & & & \end{array}$

\section{6b-3}

$\begin{array}{lrrr}\mathrm{C} & -5.51146100 & -0.09573900 & -1.27484900 \\ \mathrm{H} & -6.25725000 & 0.18172100 & -0.52221100 \\ \mathrm{H} & -5.75800500 & 0.37688600 & -2.22196800 \\ \mathrm{C} & -4.68102300 & -2.10299800 & -0.43263000 \\ \mathrm{C} & -4.09863200 & 0.18374400 & -0.77994600 \\ \mathrm{H} & -3.40540000 & 0.16978100 & -1.62266900 \\ \mathrm{C} & -3.76196600 & -3.10990800 & -1.09319200 \\ \mathrm{H} & -4.35337900 & -3.85833700 & -1.61865600 \\ \mathrm{H} & -3.14992200 & -3.60154100 & -0.33854000 \\ \mathrm{H} & -3.11262200 & -2.60583600 & -1.80744000 \\ \mathrm{C} & -5.59641800 & -2.74617100 & 0.59208600 \\ \mathrm{H} & -5.03136100 & -3.22471200 & 1.38633300 \\ \mathrm{H} & -6.19681900 & -3.48970900 & 0.07092800 \\ \mathrm{H} & -6.26107400 & -2.00613400 & 1.03659900 \\ \mathrm{O} & -5.47671900 & -1.49848300 & -1.46498400 \\ \mathrm{~N} & -3.89948200 & -0.96117100 & 0.09495600 \\ \mathrm{C} & -3.91352300 & -0.55482000 & 1.41188200 \\ \mathrm{C} & -3.80116000 & 1.38102200 & 0.14794400 \\ \mathrm{O} & -3.92178100 & -1.23755500 & 2.39691700 \\ \mathrm{O} & -3.88402100 & 0.79913100 & 1.46744600 \\ \mathrm{C} & -4.85835500 & 2.44905300 & 0.06592100 \\ \mathrm{H} & -5.79440300 & 2.18772300 & 0.54695900 \\ \mathrm{C} & -4.73238300 & 3.62154600 & -0.53369600 \\ \mathrm{H} & -5.55870900 & 4.31819400 & -0.55800500 \\ \mathrm{H} & -3.81515700 & 3.93376600 & -1.01448500 \\ \mathrm{C} & -2.38535800 & 1.91062200 & -0.06046900\end{array}$




$\begin{array}{lrrr} & & & \\ \mathrm{H} & -2.23454600 & 2.74130500 & 0.63219800 \\ \mathrm{H} & -2.32408800 & 2.31926900 & -1.07237100 \\ \mathrm{C} & -1.28025900 & 0.87816400 & 0.13416100 \\ \mathrm{H} & -1.34389600 & 0.45871000 & 1.14118600 \\ \mathrm{H} & -1.40856200 & 0.04350900 & -0.56019900 \\ \mathrm{C} & 0.10266200 & 1.48669500 & -0.06818700 \\ \mathrm{H} & 0.23849700 & 2.31705500 & 0.63124000 \\ \mathrm{H} & 0.16729600 & 1.91670800 & -1.07273900 \\ \mathrm{C} & 1.22881300 & 0.47870800 & 0.12105500 \\ \mathrm{H} & 1.09036800 & -0.35360500 & -0.57600000 \\ \mathrm{H} & 1.16527500 & 0.05054900 & 1.12590700 \\ \mathrm{C} & 2.61196800 & 1.08294000 & -0.08447900 \\ \mathrm{H} & 2.75075400 & 1.91423400 & 0.61376900 \\ \mathrm{H} & 2.67288500 & 1.51333600 & -1.08911500 \\ \mathrm{C} & 3.74047800 & 0.07654400 & 0.10048300 \\ \mathrm{H} & 3.59926200 & -0.75677500 & -0.59496900 \\ \mathrm{H} & 3.68244900 & -0.35108800 & 1.10621100 \\ \mathrm{C} & 5.12308000 & 0.67990200 & -0.11161700 \\ \mathrm{H} & 5.17967400 & 1.10857100 & -1.11721400 \\ \mathrm{H} & 5.26476800 & 1.51269600 & 0.58436000 \\ \mathrm{C} & 6.25267100 & -0.32582900 & 0.07043700 \\ \mathrm{H} & 6.10926800 & -1.15997000 & -0.62360800 \\ \mathrm{H} & 6.19834900 & -0.75253800 & 1.07685500 \\ \mathrm{C} & 7.63465100 & 0.27722400 & -0.14679600 \\ \mathrm{H} & 7.68796300 & 0.70487300 & -1.15300400 \\ \mathrm{H} & 7.77857000 & 1.11080900 & 0.54783200 \\ \mathrm{C} & 8.76481200 & -0.72802100 & 0.03268600 \\ \mathrm{H} & 8.62103400 & -1.56261500 & -0.66097500 \\ \mathrm{H} & 8.71371900 & -1.15522900 & 1.03925900 \\ \mathrm{C} & 10.14694900 & -0.12547600 & -0.18716300 \\ \mathrm{H} & 10.19539800 & 0.30144900 & -1.19235500 \\ \mathrm{H} & 10.28917100 & 0.70691200 & 0.50700700 \\ \mathrm{C} & 11.26572800 & -1.14336600 & -0.00612000 \\ \mathrm{H} & 11.15403200 & -1.97018600 & -0.70924000 \\ \mathrm{H} & 12.24667800 & -0.69710500 & -0.16687700 \\ \mathrm{H} & 11.24868300 & -1.56224200 & 1.00121100 \\ & & & \\ & & & \\ & & & \end{array}$

\section{6b-4}

$\begin{array}{lrrr}\mathrm{C} & -5.91153100 & 1.15834400 & -0.47537700 \\ \mathrm{H} & -6.06043700 & 1.44420400 & 0.57215200 \\ \mathrm{H} & -6.08190500 & 2.02099600 & -1.11411600 \\ \mathrm{C} & -6.27339100 & -1.14774000 & -0.45950500 \\ \mathrm{C} & -4.55883100 & 0.48886800 & -0.67004200 \\ \mathrm{H} & -4.29799700 & 0.46935900 & -1.72895900 \\ \mathrm{C} & -6.37540400 & -2.07655000 & -1.65260700 \\ \mathrm{H} & -7.41539200 & -2.15776300 & -1.96525100 \\ \mathrm{H} & -6.00315400 & -3.06443300 & -1.38577700 \\ \mathrm{H} & -5.78738700 & -1.68453100 & -2.48101300 \\ \mathrm{C} & -7.05701000 & -1.66086500 & 0.73325300 \\ \mathrm{H} & -6.69507600 & -2.63079300 & 1.06135200 \\ \mathrm{H} & -8.09771300 & -1.74395000 & 0.42491100 \\ \mathrm{H} & -6.99187800 & -0.96545700 & 1.56888300 \\ \mathrm{O} & -6.80484900 & 0.12844600 & -0.85690300 \\ \mathrm{~N} & -4.85332400 & -0.84607200 & -0.16644000 \\ \mathrm{C} & -4.27561200 & -1.00530700 & 1.07784000 \\ \mathrm{C} & -3.34378700 & 0.90969000 & 0.17905700 \\ \mathrm{O} & -4.43326000 & -1.89945000 & 1.85913300 \\ \mathrm{O} & -3.42556400 & 0.02388900 & 1.31587700 \\ \mathrm{C} & -3.46233700 & 2.32155900 & 0.68548300 \\ \mathrm{H} & -4.06468500 & 2.42844100 & 1.58078700 \\ \mathrm{C} & -2.93690800 & 3.38951600 & 0.10828200 \\ \mathrm{H} & -3.10663500 & 4.37704400 & 0.51376900 \\ \mathrm{H} & -2.32013100 & 3.32371000 & -0.77900500 \\ \mathrm{C} & -2.05124400 & 0.60474400 & -0.57422000 \\ \mathrm{H} & -2.00137100 & 1.25725000 & -1.44976000 \\ \mathrm{H} & -2.13428000 & -0.41690900 & -0.95715000 \\ \mathrm{C} & -0.78223600 & 0.73577900 & 0.25743100\end{array}$




$\begin{array}{lrrr}\mathrm{H} & -0.68370200 & 1.75881100 & 0.62644500 \\ \mathrm{H} & -0.86154400 & 0.09220700 & 1.13572800 \\ \mathrm{C} & 0.46203000 & 0.36167400 & -0.53913000 \\ \mathrm{H} & 0.53237600 & 0.99886800 & -1.42669900 \\ \mathrm{H} & 0.36385900 & -0.66490600 & -0.90533000 \\ \mathrm{C} & 1.74562000 & 0.48569600 & 0.27151100 \\ \mathrm{H} & 1.84592300 & 1.51307500 & 0.63511200 \\ \mathrm{H} & 1.67335500 & -0.14822800 & 1.16041900 \\ \mathrm{C} & 2.99152200 & 0.10660400 & -0.51870900 \\ \mathrm{H} & 3.06030200 & 0.73757900 & -1.41056100 \\ \mathrm{H} & 2.89198400 & -0.92227100 & -0.87847000 \\ \mathrm{C} & 4.27699100 & 0.23516000 & 0.28849800 \\ \mathrm{H} & 4.37718200 & 1.26438400 & 0.64725000 \\ \mathrm{H} & 4.20792900 & -0.39455000 & 1.18096300 \\ \mathrm{C} & 5.52252900 & -0.14598000 & -0.50142800 \\ \mathrm{H} & 5.42326800 & -1.17614000 & -0.85771800 \\ \mathrm{H} & 5.58958000 & 0.48184800 & -1.39558400 \\ \mathrm{C} & 6.80909300 & -0.01377100 & 0.30345500 \\ \mathrm{H} & 6.74232500 & -0.64132600 & 1.19769000 \\ \mathrm{H} & 6.90841400 & 1.01648000 & 0.65952900 \\ \mathrm{C} & 8.05426700 & -0.39505200 & -0.48704400 \\ \mathrm{H} & 7.95562200 & -1.42587500 & -0.84168500 \\ \mathrm{H} & 8.11977000 & 0.23143400 & -1.38225400 \\ \mathrm{C} & 9.34144000 & -0.26049000 & 0.31608500 \\ \mathrm{H} & 9.27748300 & -0.88730800 & 1.21125500 \\ \mathrm{H} & 9.44082800 & 0.77017400 & 0.67140700 \\ \mathrm{C} & 10.58708900 & -0.64077400 & -0.47475800 \\ \mathrm{H} & 10.64876300 & -0.01418400 & -1.36839900 \\ \mathrm{H} & 10.48633800 & -1.67031200 & -0.82814600 \\ \mathrm{C} & 11.86542200 & -0.49985200 & 0.34163600 \\ \mathrm{H} & 12.74632100 & -0.77448700 & -0.23787800 \\ \mathrm{H} & 11.99534100 & 0.52839600 & 0.68270200 \\ \mathrm{H} & 11.83269500 & -1.13892300 & 1.22536800 \\ & & & \\ & & & \\ & & & \end{array}$

\section{6b-5}

$\mathrm{H}$

$\mathrm{H}$

C

C

$\mathrm{H}$

C

$\mathrm{H}$

$\mathrm{H}$

$\mathrm{H}$

C

$\mathrm{H}$

$\mathrm{H}$

$\mathrm{H}$

$\mathrm{O}$

N

C

C

$\mathrm{O}$

$\mathrm{O}$

C

$\mathrm{H}$

C

$\mathrm{H}$

$\mathrm{H}$

$\mathrm{C}$

$\mathrm{H}$

$\mathrm{H}$

C

$\mathrm{H}$

$\mathrm{H}$

C

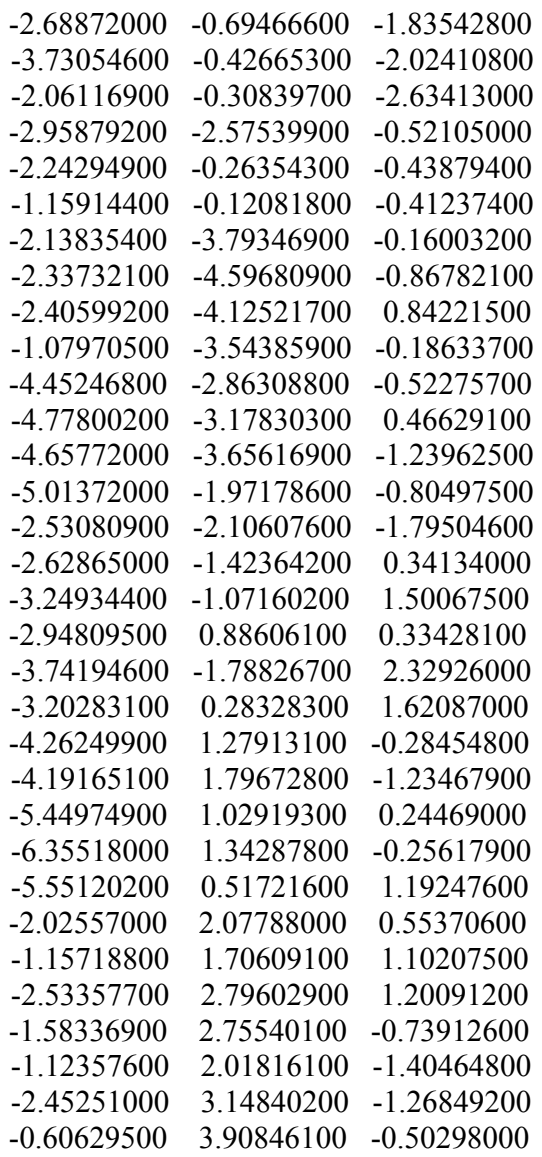




$\begin{array}{lrrr}\mathrm{H} & -1.12126100 & 4.69907400 & 0.04810200 \\ \mathrm{H} & -0.33434900 & 4.33214100 & -1.47269200 \\ \mathrm{C} & 0.67096800 & 3.52679700 & 0.24449100 \\ \mathrm{H} & 0.43902900 & 3.27003300 & 1.28149400 \\ \mathrm{H} & 1.31900600 & 4.40537600 & 0.29251600 \\ \mathrm{C} & 1.44229000 & 2.37844800 & -0.39604400 \\ \mathrm{H} & 0.84944100 & 1.45906200 & -0.35094600 \\ \mathrm{H} & 1.59553400 & 2.59130700 & -1.45946900 \\ \mathrm{C} & 2.78900000 & 2.12529000 & 0.26969300 \\ \mathrm{H} & 3.39897700 & 3.03150200 & 0.20507500 \\ \mathrm{H} & 2.63375900 & 1.93622200 & 1.33655000 \\ \mathrm{C} & 3.55258600 & 0.95838300 & -0.34317200 \\ \mathrm{H} & 2.94605400 & 0.05022300 & -0.26901000 \\ \mathrm{H} & 3.69838500 & 1.14301100 & -1.41220800 \\ \mathrm{C} & 4.90503400 & 0.71457100 & 0.31410200 \\ \mathrm{H} & 4.75959700 & 0.53354000 & 1.38365100 \\ \mathrm{H} & 5.51282200 & 1.62154000 & 0.23708100 \\ \mathrm{C} & 5.66710000 & -0.45521900 & -0.29542400 \\ \mathrm{H} & 5.06050000 & -1.36276900 & -0.21539000 \\ \mathrm{H} & 5.80951300 & -0.27571200 & -1.36577700 \\ \mathrm{C} & 7.02146000 & -0.69623400 & 0.35846500 \\ \mathrm{H} & 6.88027600 & -0.87456300 & 1.42923100 \\ \mathrm{H} & 7.62951100 & 0.21043500 & 0.27733900 \\ \mathrm{C} & 7.78340800 & -1.86746900 & -0.24922500 \\ \mathrm{H} & 7.17530800 & -2.77200600 & -0.16589800 \\ \mathrm{H} & 7.92208300 & -1.68827100 & -1.31866000 \\ \mathrm{C} & 9.13594900 & -2.09401400 & 0.41397400 \\ \mathrm{H} & 9.01527700 & -2.29914600 & 1.47876100 \\ \mathrm{H} & 9.66726100 & -2.93473000 & -0.03111800 \\ \mathrm{H} & 9.76717000 & -1.20926400 & 0.31775100 \\ & & & \end{array}$

6b-6

$\begin{array}{lrrr}\mathrm{C} & -3.80232900 & 1.37841400 & 0.73187100 \\ \mathrm{H} & -3.68869000 & 0.93481400 & 1.72344700 \\ \mathrm{H} & -3.37355800 & 2.37702500 & 0.72658600 \\ \mathrm{C} & -5.63625500 & 0.21243100 & -0.05203500 \\ \mathrm{C} & -3.24733100 & 0.46290300 & -0.35864800 \\ \mathrm{H} & -2.96092400 & 1.04778400 & -1.23641800 \\ \mathrm{C} & -6.70908100 & 0.42378700 & -1.09684700 \\ \mathrm{H} & -7.56548000 & 0.92770300 & -0.65120100 \\ \mathrm{H} & -7.02633300 & -0.54120900 & -1.48966700 \\ \mathrm{H} & -6.31568900 & 1.03158200 & -1.90898000 \\ \mathrm{C} & -6.12893800 & -0.61524700 & 1.12520500 \\ \mathrm{H} & -6.44115200 & -1.60199000 & 0.79021200 \\ \mathrm{H} & -6.97454600 & -0.10593400 & 1.58407500 \\ \mathrm{H} & -5.33827800 & -0.73476700 & 1.86671200 \\ \mathrm{O} & -5.17495500 & 1.48730300 & 0.38253900 \\ \mathrm{~N} & -4.40904900 & -0.35823800 & -0.64209400 \\ \mathrm{C} & -4.08867100 & -1.67871700 & -0.72840400 \\ \mathrm{C} & -2.16430800 & -0.61202400 & -0.06083400 \\ \mathrm{O} & -4.82465200 & -2.61487100 & -0.88550400 \\ \mathrm{O} & -2.73615400 & -1.80181200 & -0.64364700 \\ \mathrm{C} & -1.96651800 & -0.84017700 & 1.41358100 \\ \mathrm{H} & -1.48776800 & -0.02693400 & 1.94811000 \\ \mathrm{C} & -2.33841600 & -1.93108200 & 2.06435400 \\ \mathrm{H} & -2.16345200 & -2.02778200 & 3.12692400 \\ \mathrm{H} & -2.81569900 & -2.75823200 & 1.55581400 \\ \mathrm{C} & -0.85568500 & -0.32396500 & -0.78464600 \\ \mathrm{H} & -1.08326300 & -0.25858000 & -1.85151700 \\ \mathrm{H} & -0.19156200 & -1.17954100 & -0.64776400 \\ \mathrm{C} & -0.16397700 & 0.95472400 & -0.32521400 \\ \mathrm{H} & -0.86882100 & 1.79414200 & -0.34743700 \\ \mathrm{H} & 0.16011500 & 0.84724500 & 0.71150900 \\ \mathrm{C} & 1.03899700 & 1.29391100 & -1.19983800 \\ \mathrm{H} & 0.71380700 & 1.35494500 & -2.24110500 \\ \mathrm{H} & 1.75490200 & 0.46816800 & -1.15306100 \\ \mathrm{C} & 1.72560300 & 2.60336200 & -0.81647200\end{array}$




$\begin{array}{lccc}\mathrm{H} & 2.55953800 & 2.77628300 & -1.49968600 \\ \mathrm{H} & 1.02743200 & 3.42957200 & -0.97422500 \\ \mathrm{C} & 2.23129800 & 2.65665400 & 0.62746900 \\ \mathrm{H} & 1.38083400 & 2.69489600 & 1.31218800 \\ \mathrm{H} & 2.77532800 & 3.59389500 & 0.76923900 \\ \mathrm{C} & 3.13978100 & 1.49315000 & 1.02210800 \\ \mathrm{H} & 3.45425400 & 1.62840100 & 2.06016000 \\ \mathrm{H} & 2.57810400 & 0.55387300 & 0.99790600 \\ \mathrm{C} & 4.37944800 & 1.35410300 & 0.14545200 \\ \mathrm{H} & 4.08556100 & 1.13190900 & -0.88454000 \\ \mathrm{H} & 4.90810200 & 2.31263800 & 0.11273000 \\ \mathrm{C} & 5.33024200 & 0.26776400 & 0.63253700 \\ \mathrm{H} & 4.79404200 & -0.68541000 & 0.68138200 \\ \mathrm{H} & 5.64463800 & 0.49446300 & 1.65604300 \\ \mathrm{C} & 6.56199900 & 0.10732900 & -0.24919300 \\ \mathrm{H} & 6.24710600 & -0.12572100 & -1.27123200 \\ \mathrm{H} & 7.09466100 & 1.06203700 & -0.30333000 \\ \mathrm{C} & 7.51611600 & -0.97382700 & 0.24166400 \\ \mathrm{H} & 6.98361400 & -1.92856600 & 0.29859300 \\ \mathrm{H} & 7.83428300 & -0.74014800 & 1.26269100 \\ \mathrm{C} & 8.74631700 & -1.13727600 & -0.64233000 \\ \mathrm{H} & 9.27604300 & -0.18272000 & -0.69891300 \\ \mathrm{H} & 8.42651900 & -1.37170200 & -1.66101100 \\ \mathrm{C} & 9.69081500 & -2.22069900 & -0.13767400 \\ \mathrm{H} & 10.56438700 & -2.32517000 & -0.78043200 \\ \mathrm{H} & 10.04108500 & -1.99037000 & 0.86966900 \\ \mathrm{H} & 9.18565900 & -3.18699200 & -0.09856000\end{array}$

\section{6b-7}

\begin{tabular}{|c|c|c|c|}
\hline $\mathrm{C}$ & -0.84762700 & -2.16053000 & 1.76903900 \\
\hline $\mathrm{H}$ & -1.38224600 & -3.03640300 & 1.39272000 \\
\hline $\mathrm{H}$ & -0.09294400 & -2.47098800 & 2.48730500 \\
\hline $\mathrm{C}$ & -2.43569800 & -0.50961300 & 1.43165700 \\
\hline $\mathrm{C}$ & -0.30489900 & -1.31024900 & 0.62366900 \\
\hline $\mathrm{H}$ & 0.51278800 & -0.68072400 & 0.98418400 \\
\hline $\mathrm{C}$ & -2.65539700 & 0.88738700 & 1.96836300 \\
\hline $\mathrm{H}$ & -3.29435300 & 0.85082200 & 2.84963900 \\
\hline $\mathrm{H}$ & -3.13097900 & 1.50056800 & 1.20281900 \\
\hline $\mathrm{H}$ & -1.69721200 & 1.33112800 & 2.23363900 \\
\hline $\mathrm{C}$ & -3.73602300 & -1.20242400 & 1.05834100 \\
\hline $\mathrm{H}$ & -4.26363700 & -0.64180500 & 0.29066200 \\
\hline $\mathrm{H}$ & -4.35857000 & -1.27190800 & 1.94852200 \\
\hline $\mathrm{H}$ & -3.53964800 & -2.20724100 & 0.68265000 \\
\hline $\mathrm{O}$ & -1.73376000 & -1.26221900 & 2.41968600 \\
\hline $\mathrm{N}$ & -1.47064400 & -0.50815700 & 0.31343100 \\
\hline $\mathrm{C}$ & -1.77090900 & -0.52716400 & -1.01475800 \\
\hline $\mathrm{C}$ & 0.05216000 & -1.92346800 & -0.76118100 \\
\hline $\mathrm{O}$ & -2.70625300 & -0.01786200 & -1.57165200 \\
\hline $\mathrm{O}$ & -0.79394700 & -1.19795200 & -1.67604000 \\
\hline $\mathrm{C}$ & -0.26743000 & -3.38956100 & -0.84743800 \\
\hline $\mathrm{H}$ & 0.33244600 & -4.02657500 & -0.20550400 \\
\hline $\mathrm{C}$ & -1.19958000 & -3.91610600 & -1.62494900 \\
\hline $\mathrm{H}$ & -1.37096300 & -4.98346000 & -1.63884400 \\
\hline $\mathrm{H}$ & -1.80748400 & -3.29815400 & -2.27189500 \\
\hline $\mathrm{C}$ & 1.49485200 & -1.61628400 & -1.14735400 \\
\hline $\mathrm{H}$ & 1.59377700 & -0.52964100 & -1.11050600 \\
\hline $\mathrm{H}$ & 1.64521600 & -1.91688200 & -2.18622400 \\
\hline $\mathrm{C}$ & 2.53928600 & -2.27504600 & -0.25208200 \\
\hline $\mathrm{H}$ & 2.28011500 & -2.12357200 & 0.80019000 \\
\hline $\mathrm{H}$ & 2.53523400 & -3.35383600 & -0.41535800 \\
\hline $\mathrm{C}$ & 3.95432000 & -1.74880800 & -0.51073900 \\
\hline $\mathrm{H}$ & 4.25311400 & -2.02869100 & -1.52385100 \\
\hline $\mathrm{H}$ & 4.64205500 & -2.25848800 & 0.16823600 \\
\hline $\mathrm{C}$ & 4.11669300 & -0.23720000 & -0.34295000 \\
\hline $\mathrm{H}$ & 5.16131500 & 0.02611500 & -0.52883200 \\
\hline $\mathrm{H}$ & 3.53997800 & 0.28210600 & -1.11104000 \\
\hline $\mathrm{C}$ & 3.72017300 & 0.27386300 & 1.03814300 \\
\hline
\end{tabular}




$\begin{array}{lrrr}\mathrm{H} & 4.30952100 & -0.25505000 & 1.79257900 \\ \mathrm{H} & 2.67645900 & 0.02325000 & 1.24407200 \\ \mathrm{C} & 3.91412300 & 1.77996500 & 1.21296600 \\ \mathrm{H} & 3.67193000 & 2.04888900 & 2.24448800 \\ \mathrm{H} & 4.97068500 & 2.02097800 & 1.06928300 \\ \mathrm{C} & 3.07132200 & 2.64390000 & 0.27266400 \\ \mathrm{H} & 3.27734600 & 3.69549000 & 0.48787400 \\ \mathrm{H} & 3.37928500 & 2.48438900 & -0.76415000 \\ \mathrm{C} & 1.56953800 & 2.40623300 & 0.39152700 \\ \mathrm{H} & 1.26613500 & 2.51778300 & 1.43875200 \\ \mathrm{H} & 1.32828400 & 1.37501900 & 0.11447800 \\ \mathrm{C} & 0.73952100 & 3.33861500 & -0.48234300 \\ \mathrm{H} & 0.98291700 & 4.37833000 & -0.24253600 \\ \mathrm{H} & 1.01937100 & 3.19109500 & -1.53027500 \\ \mathrm{C} & -0.75849300 & 3.11604200 & -0.32292600 \\ \mathrm{H} & -1.04548500 & 3.32656400 & 0.71291200 \\ \mathrm{H} & -0.99004400 & 2.05908000 & -0.49057700 \\ \mathrm{C} & -1.61057400 & 3.96080000 & -1.26054100 \\ \mathrm{H} & -1.32643800 & 3.74331000 & -2.29323600 \\ \mathrm{H} & -1.39311600 & 5.01960800 & -1.09514400 \\ \mathrm{C} & -3.09923600 & 3.69603000 & -1.07329900 \\ \mathrm{H} & -3.70508600 & 4.29260700 & -1.75493100 \\ \mathrm{H} & -3.32211400 & 2.64276400 & -1.25303000 \\ \mathrm{H} & -3.40820300 & 3.93660000 & -0.05390500\end{array}$

\section{6b-8}

$3.73295200 \quad-1.67462200 \quad-1.24608800$

$3.46568900 \quad-2.45445400 \quad-0.52316400$

$\begin{array}{lll}3.34962700 & -1.94755100 & -2.22593400\end{array}$

$\begin{array}{lll}5.59438100 & -0.63695500 & -0.29019500\end{array}$

$\begin{array}{lll}3.28311600 & -0.30865200 & -0.74964600\end{array}$

$\begin{array}{llll}3.27422100 & 0.41191300 & -1.56870600\end{array}$

$\begin{array}{llll}6.48394100 & 0.41303500 & -0.92533900\end{array}$

$\begin{array}{lll}7.31559000 & -0.07173200 & -1.43453700\end{array}$

$\begin{array}{llll}6.87337700 & 1.08150200 & -0.15904300\end{array}$

$\begin{array}{llll}5.91614100 & 0.99331700 & -1.65100500\end{array}$

$\begin{array}{lll}6.33308000 & -1.45809000 & 0.74893300\end{array}$

$\begin{array}{lll}6.70870200 & -0.83591400 & 1.55593200\end{array}$

$\begin{array}{llll}7.16609900 & -1.94413300 & 0.24398500\end{array}$

$\begin{array}{lll}5.68395300 & -2.22270100 & 1.17297700\end{array}$

$\begin{array}{lll}5.13566900 & -1.50924100 & -1.33785700\end{array}$

$\begin{array}{lll}4.34485200 & -0.01543200 & 0.20496200\end{array}$

$\begin{array}{lll}3.86523800 & -0.17648900 & 1.49008900\end{array}$

$\begin{array}{lll}2.01400500 & -0.18247800 & 0.11024300\end{array}$

$\begin{array}{lll}4.48166100 & -0.17604300 & 2.51711100\end{array}$

$\begin{array}{lll}2.51707900 & -0.31205300 & 1.45431800\end{array}$

$\begin{array}{lll}1.01180900 & -1.28399400 & -0.12666400\end{array}$

$\begin{array}{llll}0.81849100 & -1.92687900 & 0.72414900\end{array}$

$\begin{array}{lll}0.39451100 & -1.48002500 & -1.28061500\end{array}$

$\begin{array}{lll}-0.31515100 & -2.28538000 & -1.40654200\end{array}$

$\begin{array}{llll}0.57548200 & -0.84669600 & -2.14227300\end{array}$

$\begin{array}{llll}1.39560400 & 1.20650400 & -0.04668800\end{array}$

$\begin{array}{lll}1.03348600 & 1.28112100 & -1.07505000\end{array}$

$\begin{array}{lll}2.19069300 & 1.95036400 & 0.06812800\end{array}$

$\begin{array}{llll}0.26926500 & 1.48624000 & 0.93967800\end{array}$

$\begin{array}{lll}-0.49928700 & 0.71631200 & 0.83357100\end{array}$

$\begin{array}{lll}0.65759500 & 1.39950700 & 1.95481600\end{array}$

$\begin{array}{lll}-0.35544500 & 2.86800400 & 0.75204500\end{array}$

$\begin{array}{llll}0.39463700 & 3.63360400 & 0.96554700\end{array}$

$\begin{array}{lll}-1.14214400 & 2.99763800 & 1.49766500\end{array}$

$\begin{array}{llll}-0.93011200 & 3.11576000 & -0.64585700\end{array}$

$\begin{array}{llll}-0.11352800 & 3.22631100 & -1.36324800\end{array}$

$\begin{array}{lll}-1.45695900 & 4.07326000 & -0.64105300\end{array}$

$\begin{array}{lll}-1.88195800 & 2.02609500 & -1.13774900\end{array}$

$\begin{array}{lll}-2.23248400 & 2.28838300 & -2.13939400\end{array}$

$\begin{array}{llll}-1.34205100 & 1.07897200 & -1.24431200\end{array}$

$\begin{array}{llll}-3.09019400 & 1.80637300 & -0.23464700\end{array}$ 


$\begin{array}{lccc}\mathrm{H} & -2.76093000 & 1.49978600 & 0.76228400 \\ \mathrm{H} & -3.62105400 & 2.75511300 & -0.10358800 \\ \mathrm{C} & -4.05106800 & 0.75722200 & -0.77995900 \\ \mathrm{H} & -4.38778800 & 1.05720400 & -1.77728700 \\ \mathrm{H} & -3.51382100 & -0.18826200 & -0.90927900 \\ \mathrm{C} & -5.26336300 & 0.52929600 & 0.11365200 \\ \mathrm{H} & -5.79878200 & 1.47503000 & 0.24370600 \\ \mathrm{H} & -4.92638600 & 0.22893300 & 1.11070000 \\ \mathrm{C} & -6.22311400 & -0.52117400 & -0.43066900 \\ \mathrm{H} & -6.55845000 & -0.22199000 & -1.42874900 \\ \mathrm{H} & -5.68847600 & -1.46778200 & -0.55839300 \\ \mathrm{C} & -7.43705200 & -0.74564300 & 0.46143000 \\ \mathrm{H} & -7.97284800 & 0.20043000 & 0.58874600 \\ \mathrm{H} & -7.10270900 & -1.04404500 & 1.46015500 \\ \mathrm{C} & -8.39732600 & -1.79698100 & -0.08111700 \\ \mathrm{H} & -8.72964300 & -1.49765200 & -1.07859900 \\ \mathrm{H} & -7.86080500 & -2.74112400 & -0.20674100 \\ \mathrm{C} & -9.60577800 & -2.00837900 & 0.82188800 \\ \mathrm{H} & -10.16896200 & -1.08113300 & 0.93806800 \\ \mathrm{H} & -10.28172100 & -2.76292700 & 0.42066800 \\ \mathrm{H} & -9.29398500 & -2.33190900 & 1.81614400\end{array}$




\section{References}

(1) Weiler, S.; Braendlin, N.; Beerli, C.; Bergsdorf, C.; Schubart, A.; Srinivas, H.; Oberhauser, B.; Billich, A. Orally Active 7-Substituted (4-Benzyl-Phthalazin-1-Y1)-2Methyl-Piperazin-1-Y1]-Nicotinonitriles as Active-Site Inhibitors of Sphingosine-1Phosphate Lyase for the Treatment of Multiple Sclerosis. J. Med. Chem. 2014, 57 (12), 5074-5084.

(2) Shao, Y.; Molnar, L. F.; Jung, Y.; Kussmann, J.; Ochsenfeld, C.; Brown, S. T.; Gilbert, A. T. B.; Slipchenko, L. V; Levchenko, S. V; O’Neill, D. P.; et al. Advances in Methods and Algorithms in a Modern Quantum Chemistry Program Package. Phys. Chem. Chem. Phys. 2006, 8 (27), 3172-3191.

(3) Becke, A. D. Density-functional Thermochemistry. III. The Role of Exact Exchange. J. Chem. Phys. 1993, 98 (7), 5648-5652.

(4) Lee, C.; Yang, W.; Parr, R. G. Development of the Colle-Salvetti Correlation-Energy Formula into a Functional of the Electron Density. Phys. Rev. B 1988, 37 (2), 785-789.

(5) Zhao, Y.; Truhlar, D. G. The M06 Suite of Density Functionals for Main Group Thermochemistry, Thermochemical Kinetics, Noncovalent Interactions, Excited States, and Transition Elements: Two New Functionals and Systematic Testing of Four M06Class Functionals and 12 Other Function. Theor. Chem. Acc. 2008, 120 (1), 215-241.

(6) Petersson, G. A.; Bennett, A.; Tensfeldt, T. G.; Al-Laham, M. A.; Shirley, W. A.; Mantzaris, J. A Complete Basis Set Model Chemistry. I. The Total Energies of Closedshell Atoms and Hydrides of the First-row Elements. J. Chem. Phys. 1988, 89 (4), 2193-2218.

(7) Petersson, G. A.; Al-Laham, M. A. A Complete Basis Set Model Chemistry. II. Openshell Systems and the Total Energies of the First-row Atoms. J. Chem. Phys. 1991, 94 (9), 6081-6090.

(8) Miertuš, S.; Scrocco, E.; Tomasi, J. Electrostatic Interaction of a Solute with a Continuum. A Direct Utilizaion of AB Initio Molecular Potentials for the Prevision of Solvent Effects. Chem. Phys. 1981, 55 (1), 117-129.

(9) Tomasi, J.; Mennucci, B.; Cammi, R. Quantum Mechanical Continuum Solvation Models. Chem. Rev. 2005, 105 (8), 2999-3094.

(10) Mennucci, B.; Cancès, E.; Tomasi, J. Evaluation of Solvent Effects in Isotropic and Anisotropic Dielectrics and in Ionic Solutions with a Unified Integral Equation Method: Theoretical Bases, Computational Implementation, and Numerical Applications. J. Phys. Chem. B 1997, 101 (49), 10506-10517.

(11) Cancès, E.; Mennucci, B.; Tomasi, J. A New Integral Equation Formalism for the Polarizable Continuum Model: Theoretical Background and Applications to Isotropic and Anisotropic Dielectrics. J. Chem. Phys. 1997, 107 (8), 3032-3041.

(12) Cancès, E.; Mennucci, B. New Applications of Integral Equations Methods for Solvation Continuum Models: Ionic Solutions and Liquid Crystals. J. Math. Chem. 1998, 23 (3), 309-326.

(13) Kendall, R. A.; Dunning, T. H.; Harrison, R. J. Electron Affinities of the First-row 
Atoms Revisited. Systematic Basis Sets and Wave Functions. J. Chem. Phys. 1992, 96 (9), 6796-6806.

(14) Frisch, M. J.; Trucks, G. W.; Schlegel, H. B.; Scuseria, G. E.; Robb, M. A.; Cheeseman, J. R.; Scalmani, G.; Barone, V.; Mennucci, B.; Petersson, G. A.; et al. Gaussian 09, Revision B.01. Gaussian 09, Revision B.01, Gaussian, Inc., Wallingford CT. Wallingford CT 2009.

(15) Byrd, J. N.; Bartlett, R. J.; Montgomery, J. A. At What Chain Length Do Unbranched Alkanes Prefer Folded Conformations? J. Phys. Chem. A 2014, 118 (9), 1706-1712.

(16) Wagner, J. P.; Schreiner, P. R. London Dispersion in Molecular ChemistryReconsidering Steric Effects. Angew. Chem. Int. Ed. 2015, 54 (42), 12274-12296.

(17) Li, A.; Muddana, H. S.; Gilson, M. K. Quantum Mechanical Calculation of Noncovalent Interactions: A Large-Scale Evaluation of PMx, DFT, and SAPT Approaches. J. Chem. Theory Comput. 2014, 10 (4), 1563-1575.

(18) Dennington, R.; Keith, T. A.; Millam, J. M. GaussView \{V\}ersion \{6\}. 2016.

(19) Debie, E.; De Gussem, E.; Dukor, R. K.; Herrebout, W.; Nafie, L. a.; Bultinck, P. A Confidence Level Algorithm for the Determination of Absolute Configuration Using Vibrational Circular Dichroism or Raman Optical Activity. ChemPhysChem 2011, 12 (8), 1542-1549.

(20) Vandenbussche, J.; Bultinck, P.; Przybył, A. K.; Herrebout, W. A. Statistical Validation of Absolute Configuration Assignment in Vibrational Optical Activity. $J$. Chem. Theory Comput. 2013, 9 (12), 5504-5512.

(21) NIST Standard Reference Database -Computational Chemistry Comparison and Benchmark DataBase. 2018.

(22) Stephens, P. J.; Devlin, F. J.; Cheeseman, J. R. VCD Spectroscopy for Organic Chemists; CRC Press: Boca Raton, CA, 2012. 


\section{Copies of NMR spectra}

Copies of the NMR spectra of all the synthesized compounds can be found in the following pages. 
<smiles>CCCCCCCCCCCCCCCCCCC(=O)OC(C)(C)C</smiles>

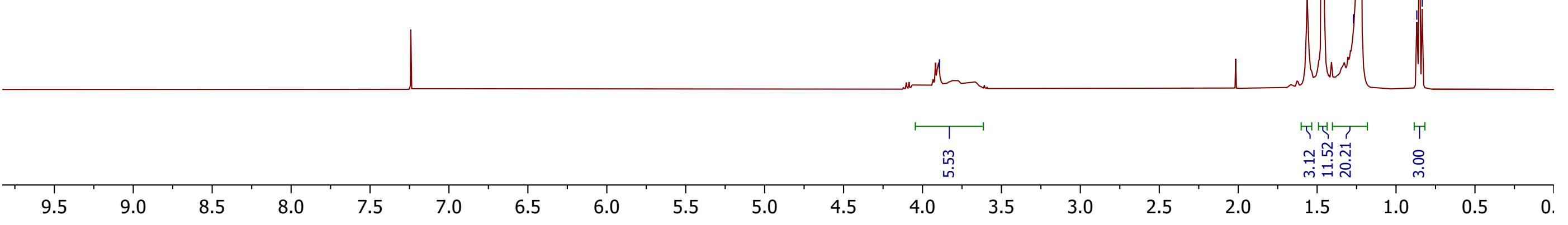



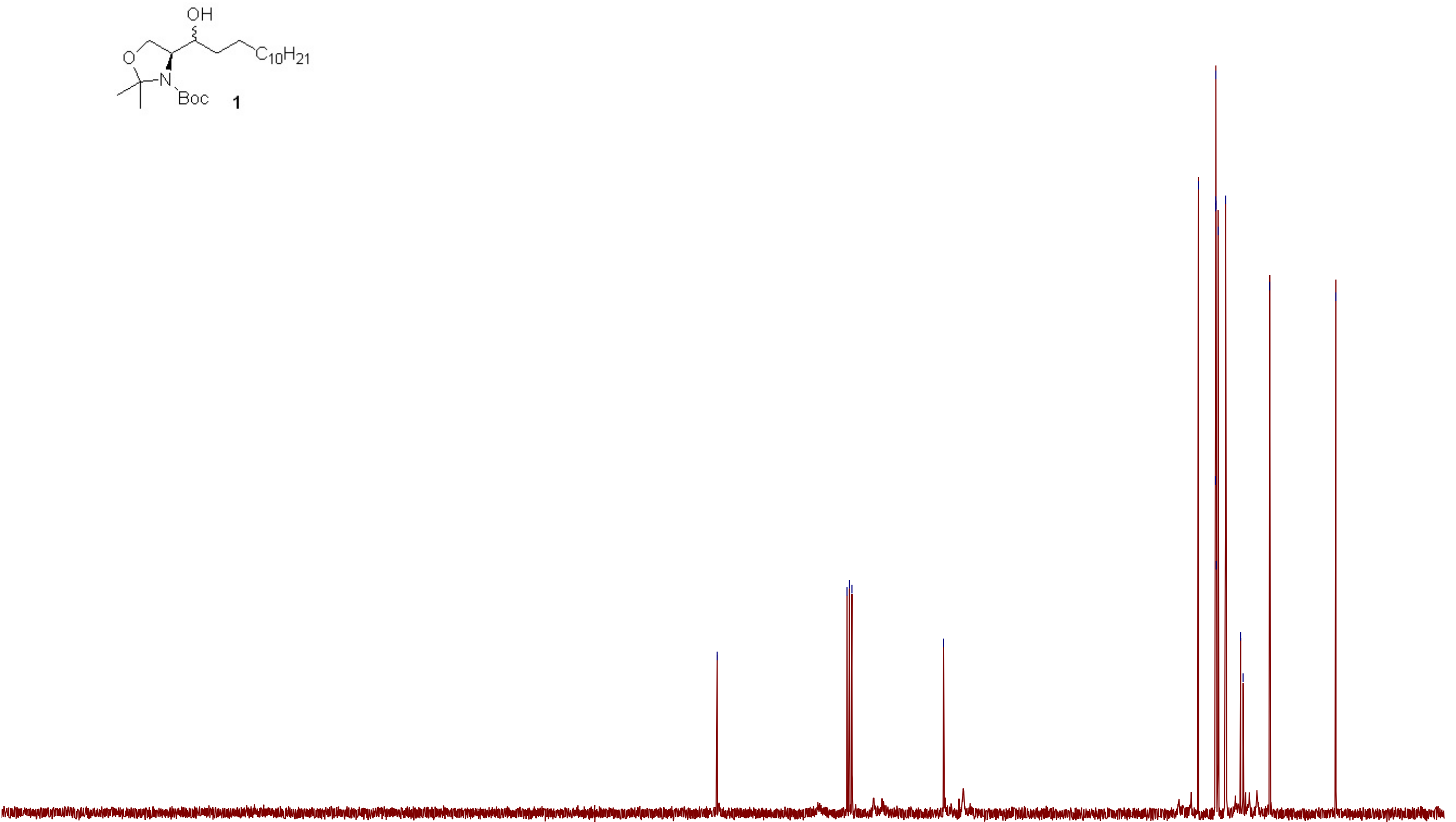

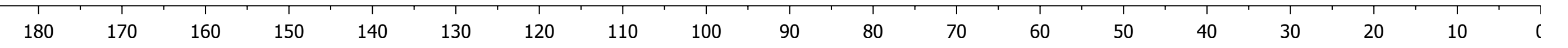




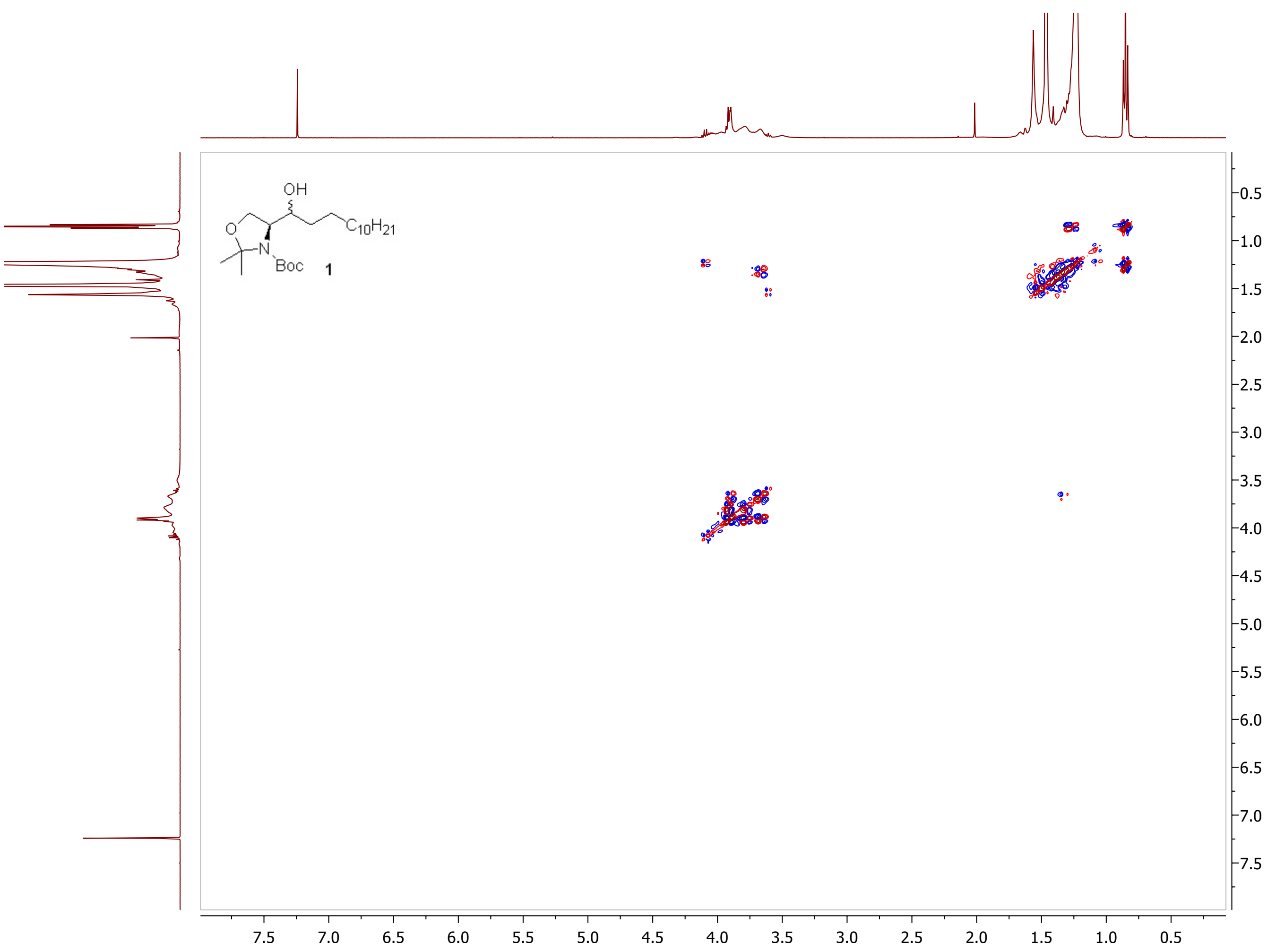



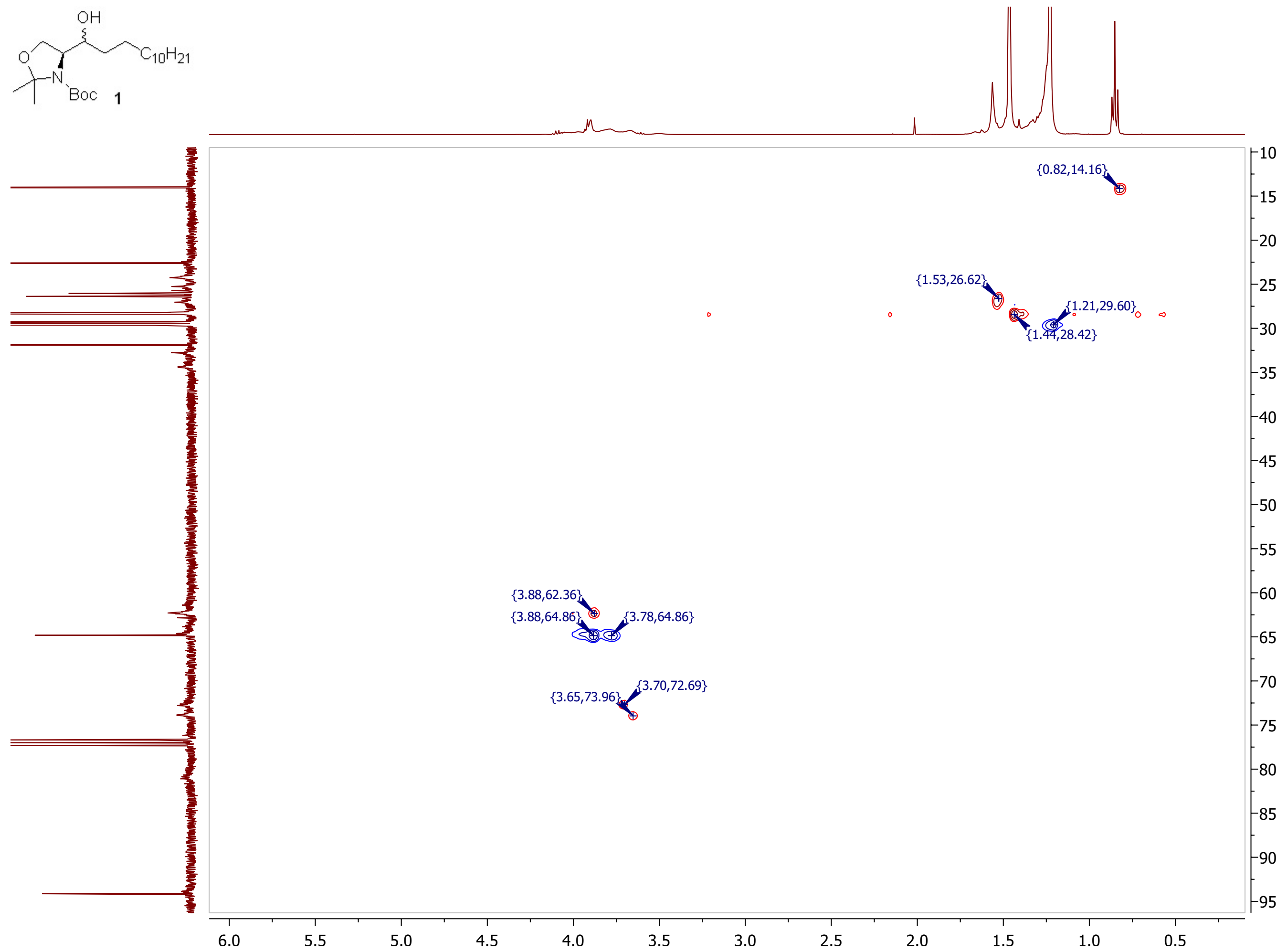


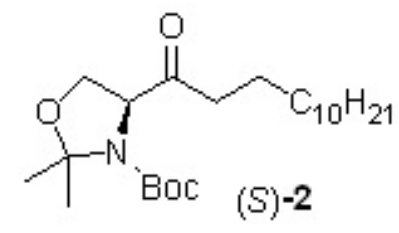

$(S)-2$

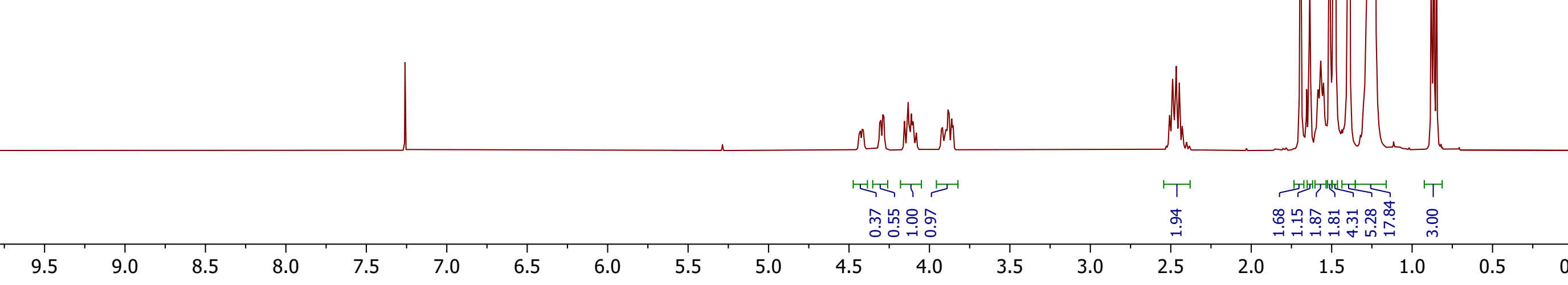



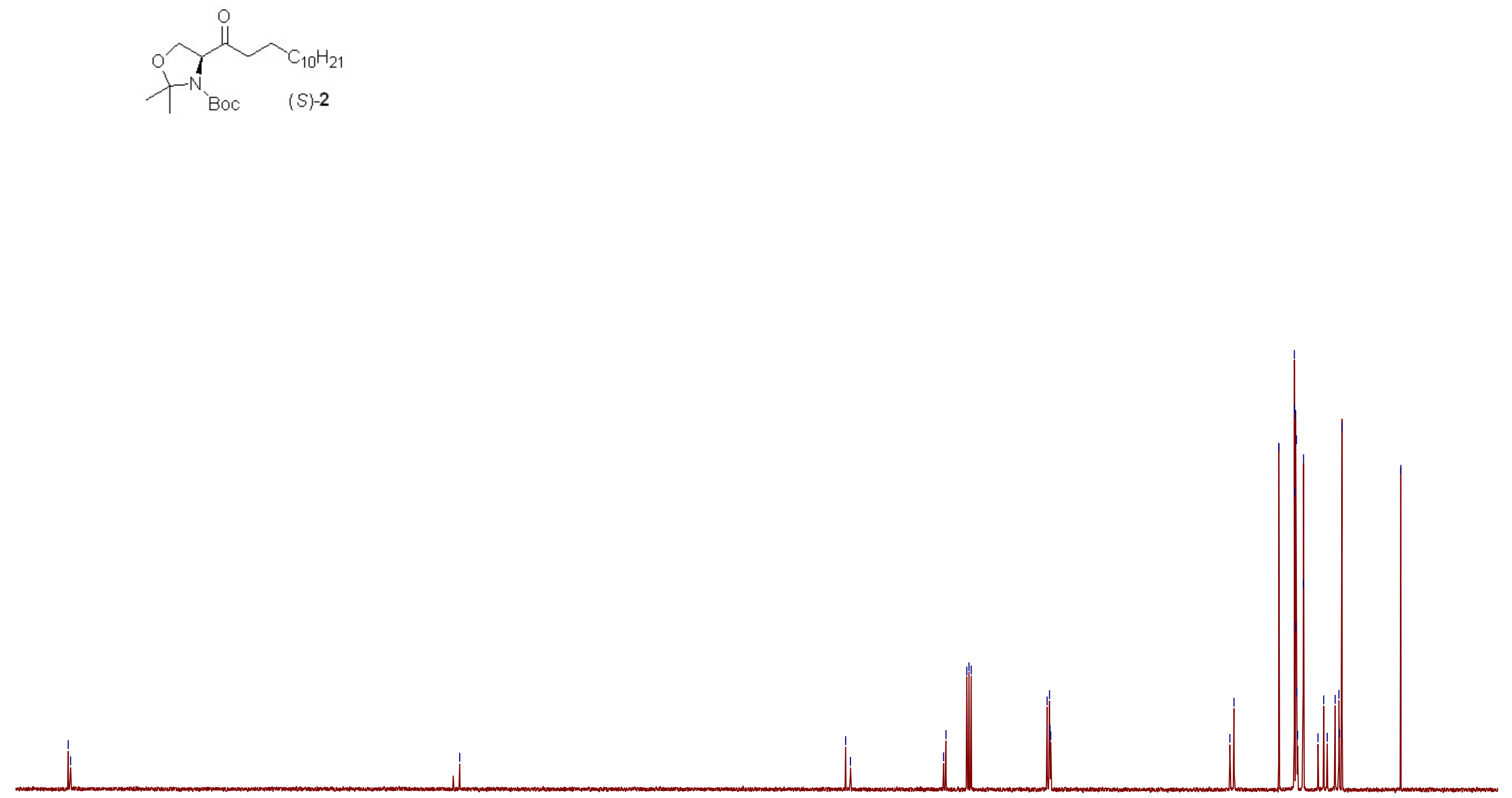

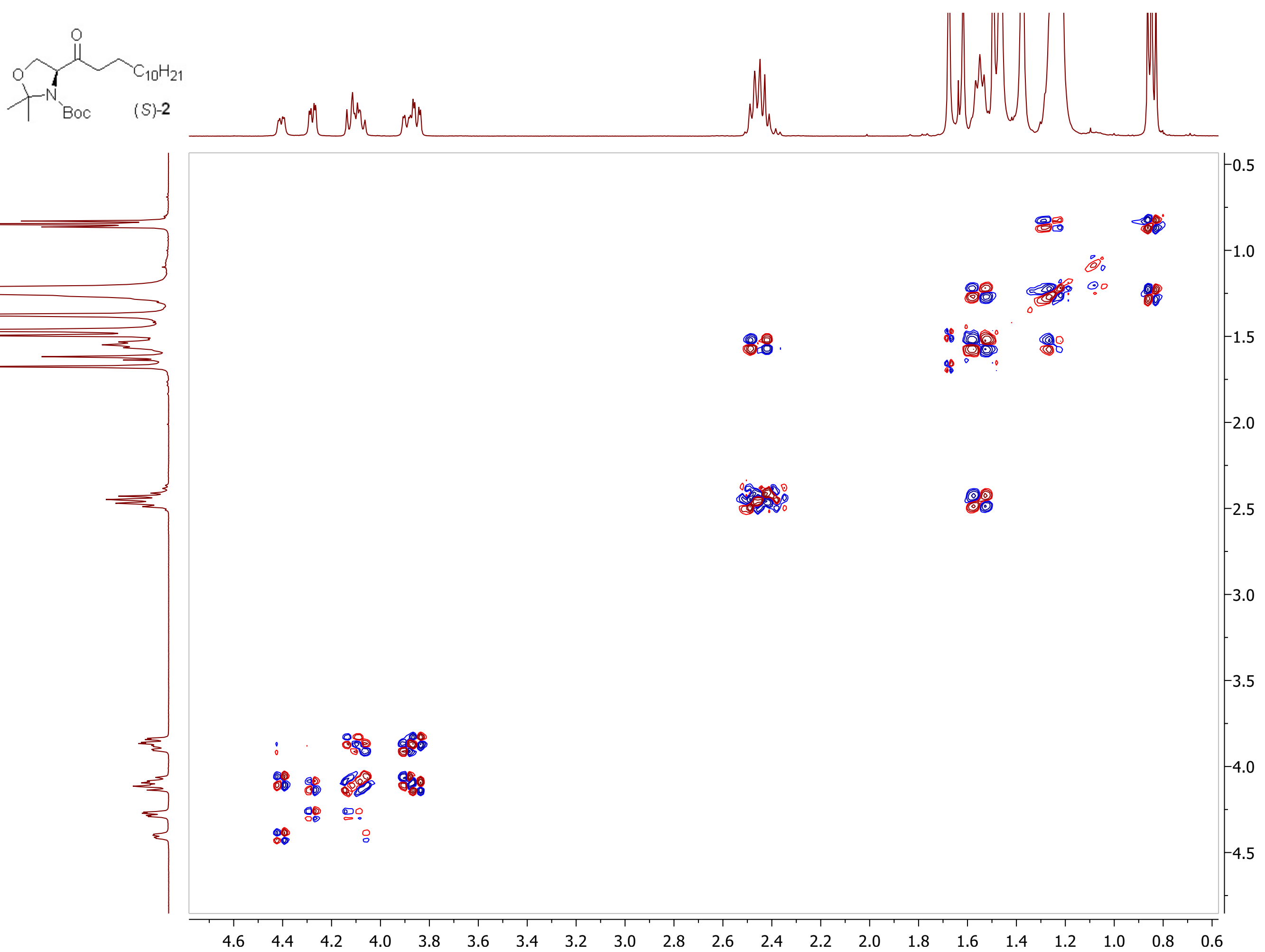

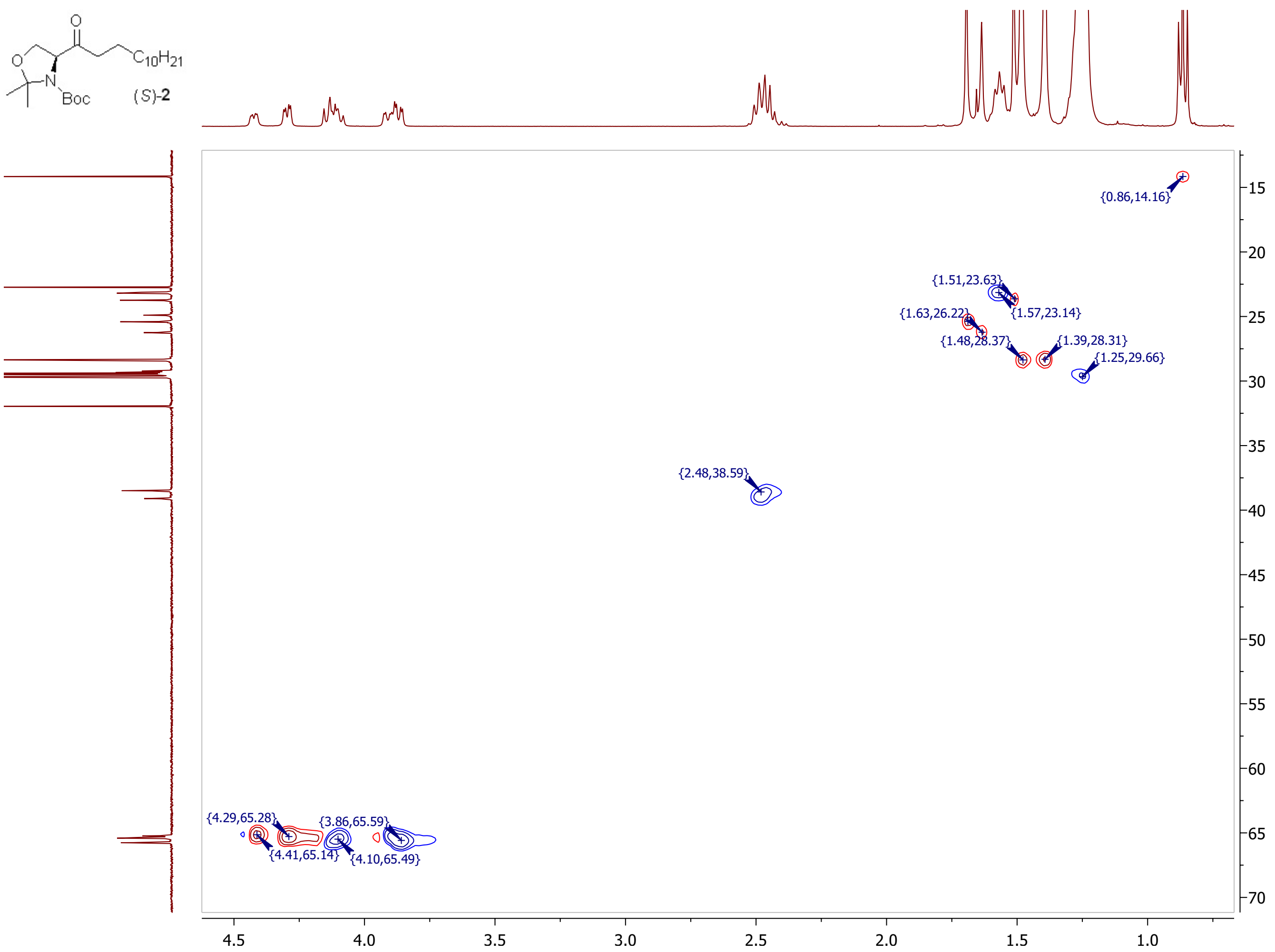


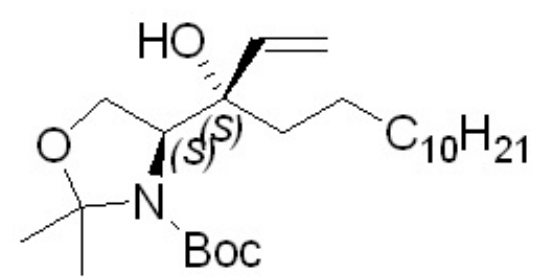

3a $(2 S, 3 S)$

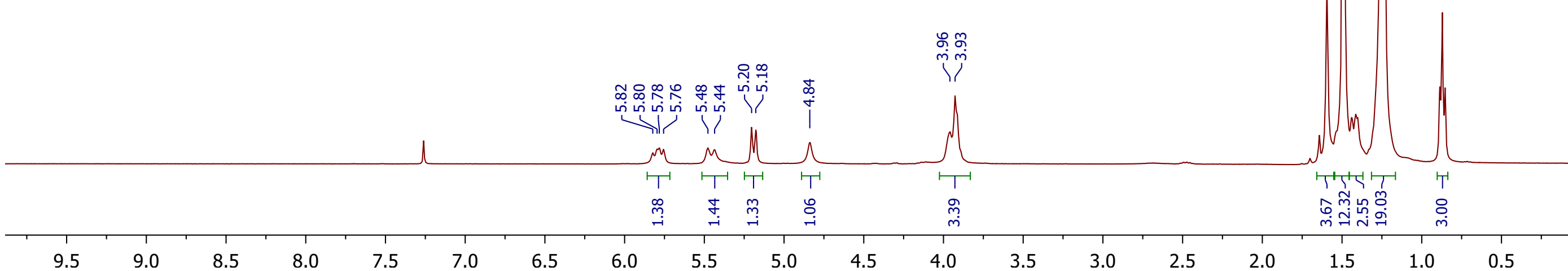




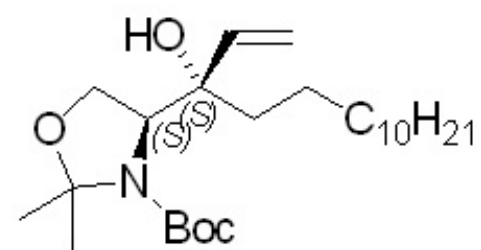

$3 a(2 S, 3 S)$

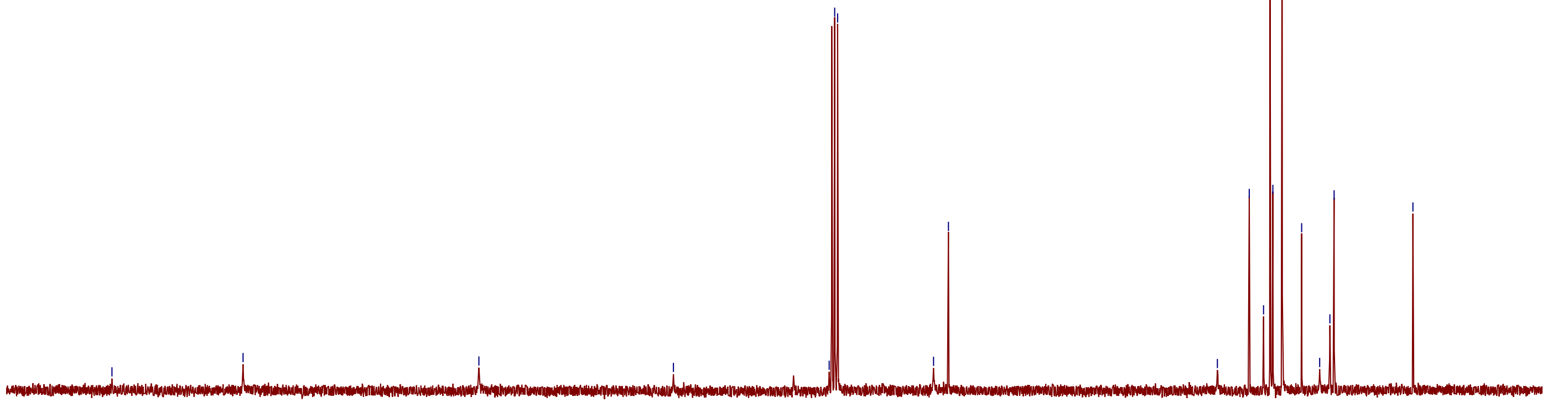




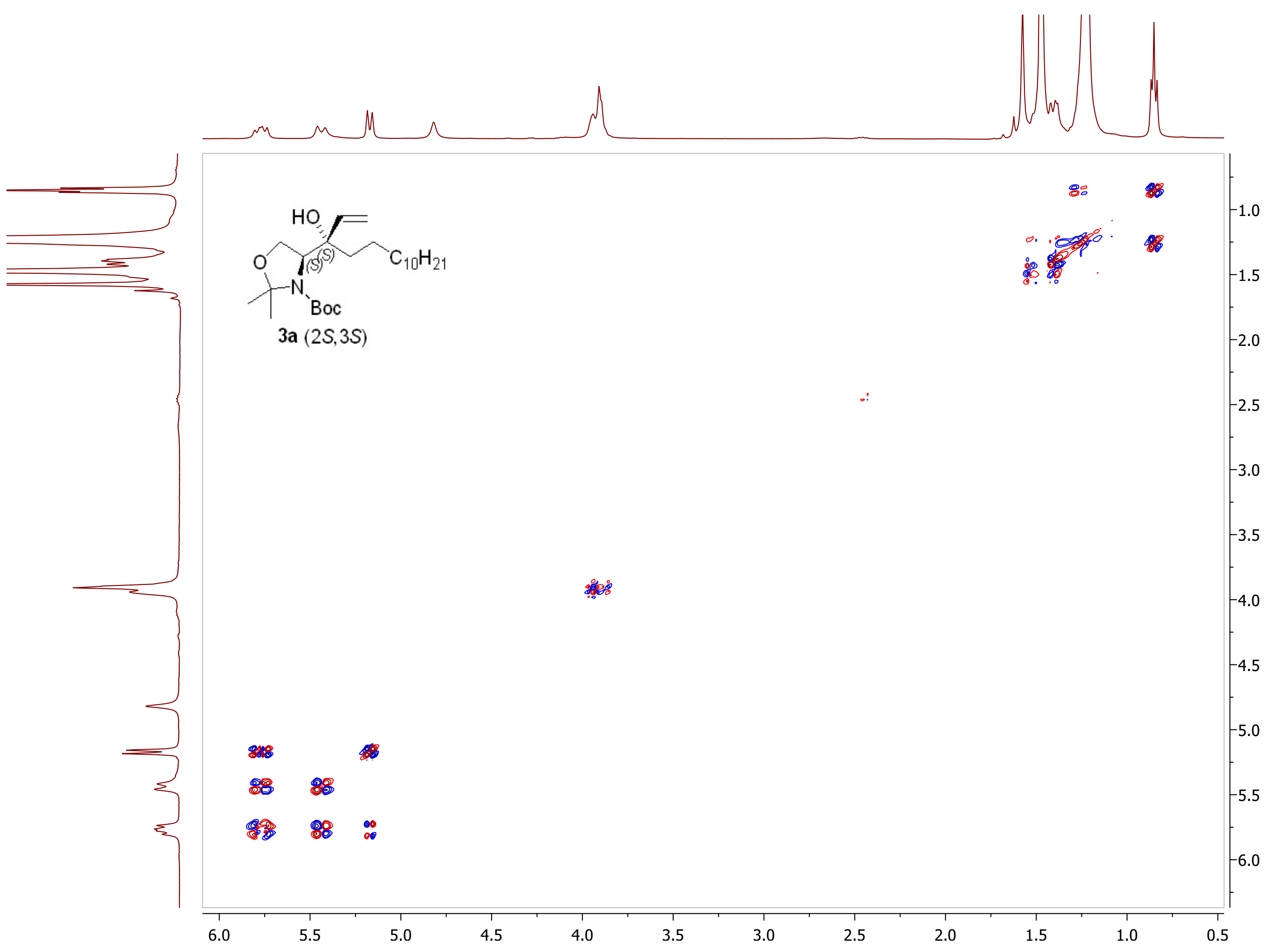




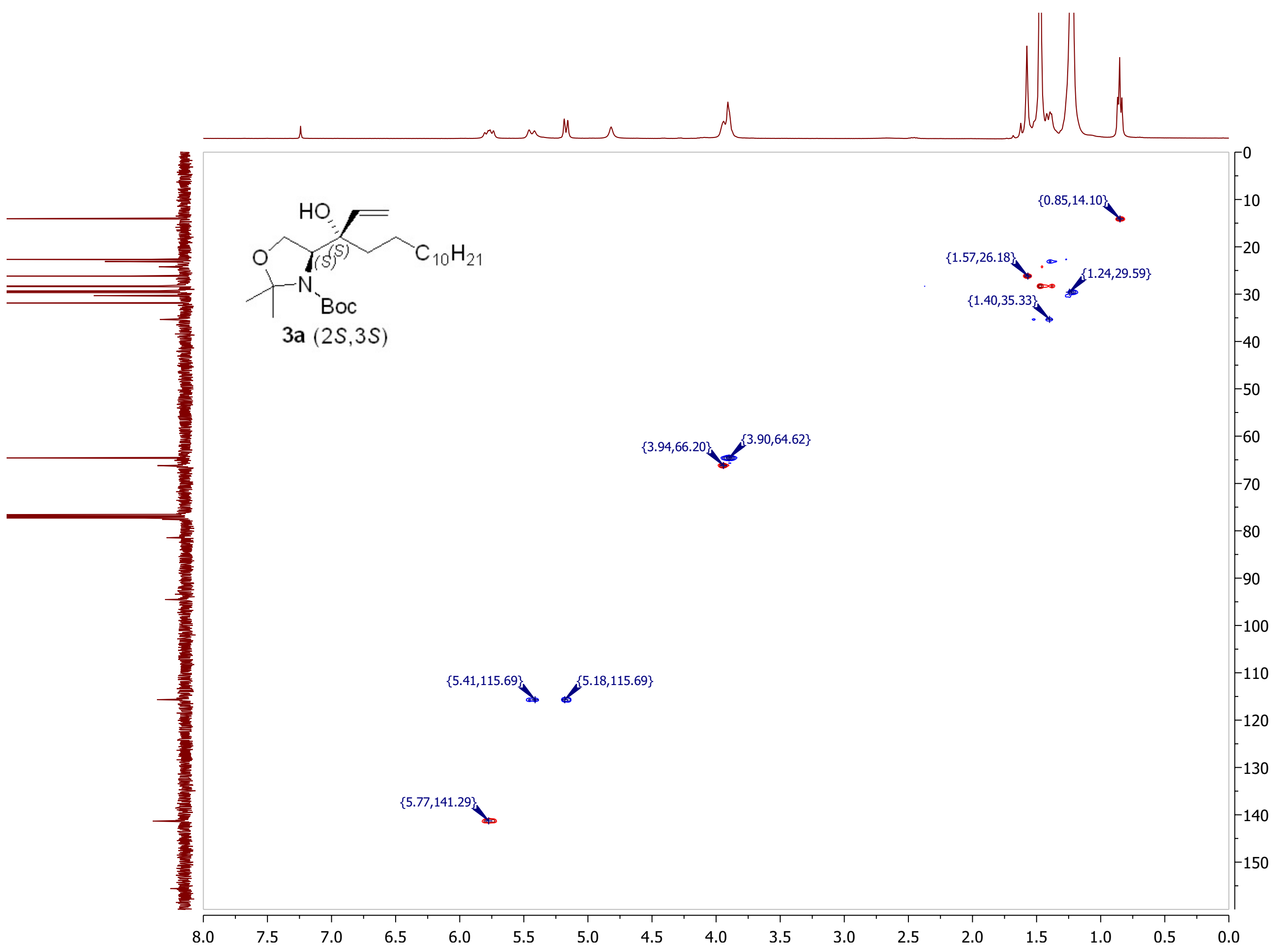


$\overbrace{\mathrm{BOC}}^{\mathrm{NO}(\mathrm{S})^{R}} \mathrm{C}_{10} \mathrm{H}_{21}$

3b $(2 S, 3 R)$

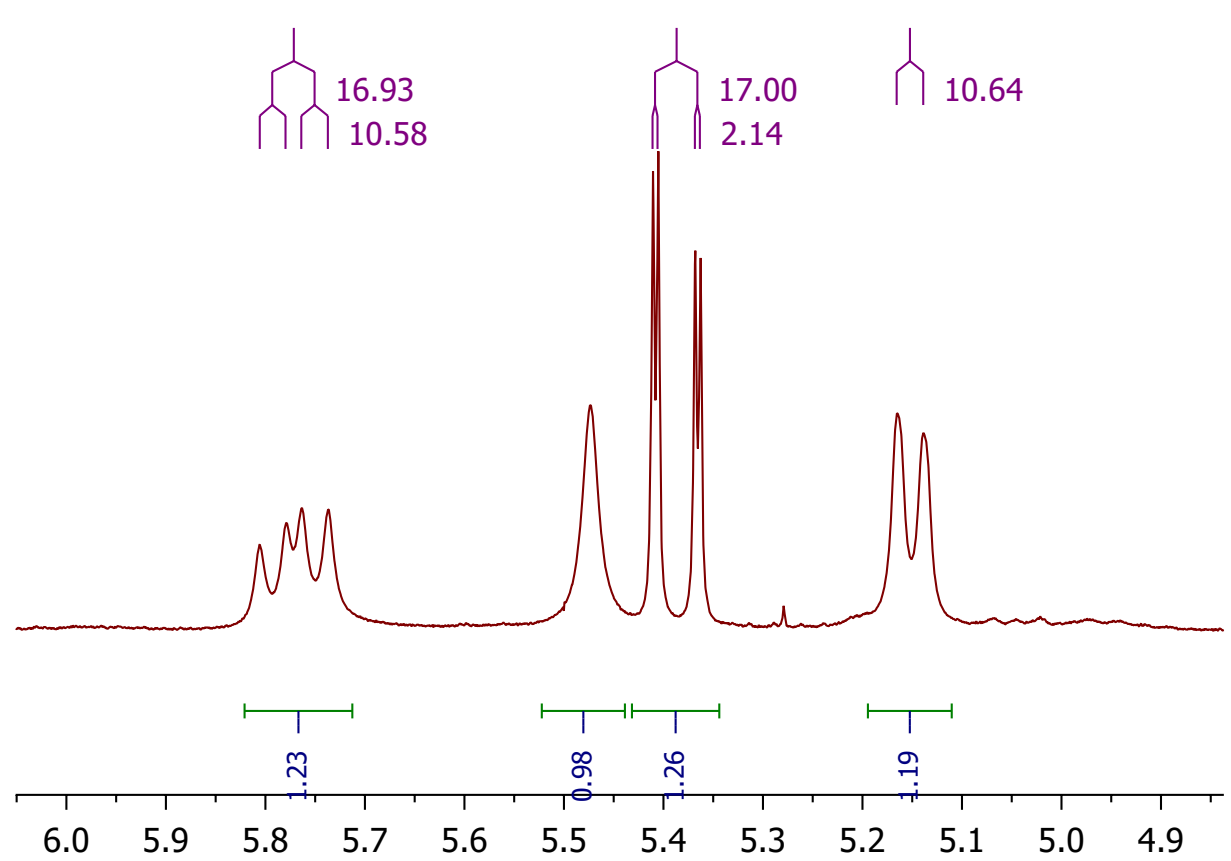

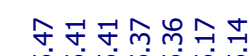

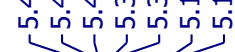

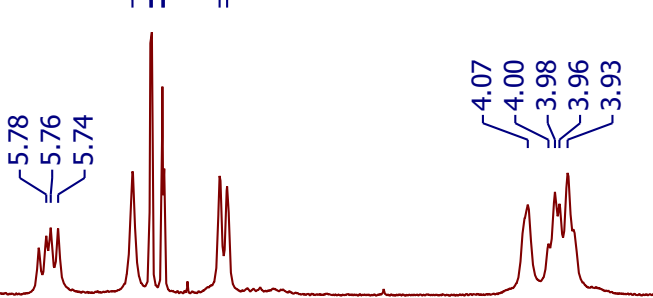

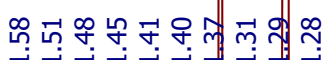

ifi-i-iti

10

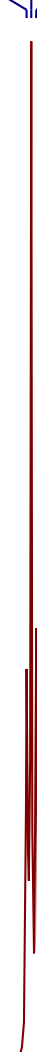

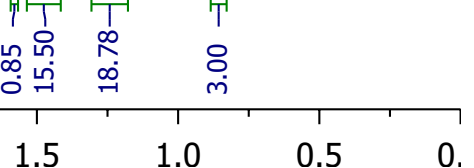

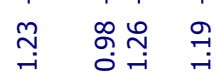

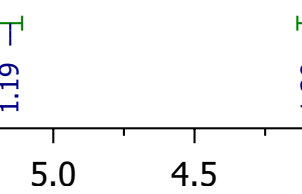

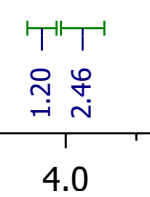




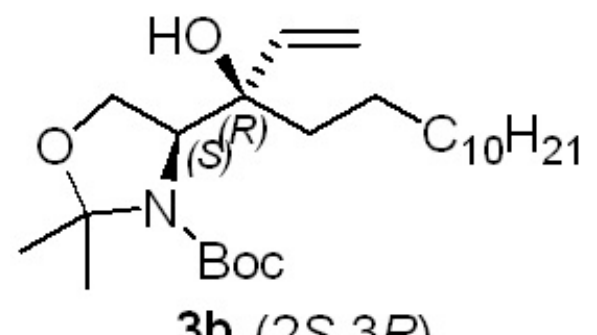

3b $(2 S, 3 R)$

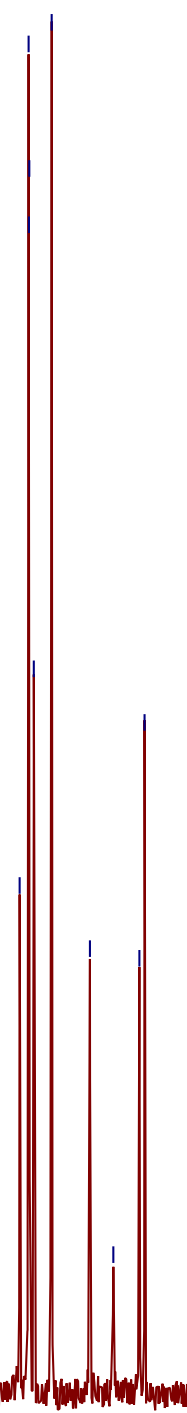




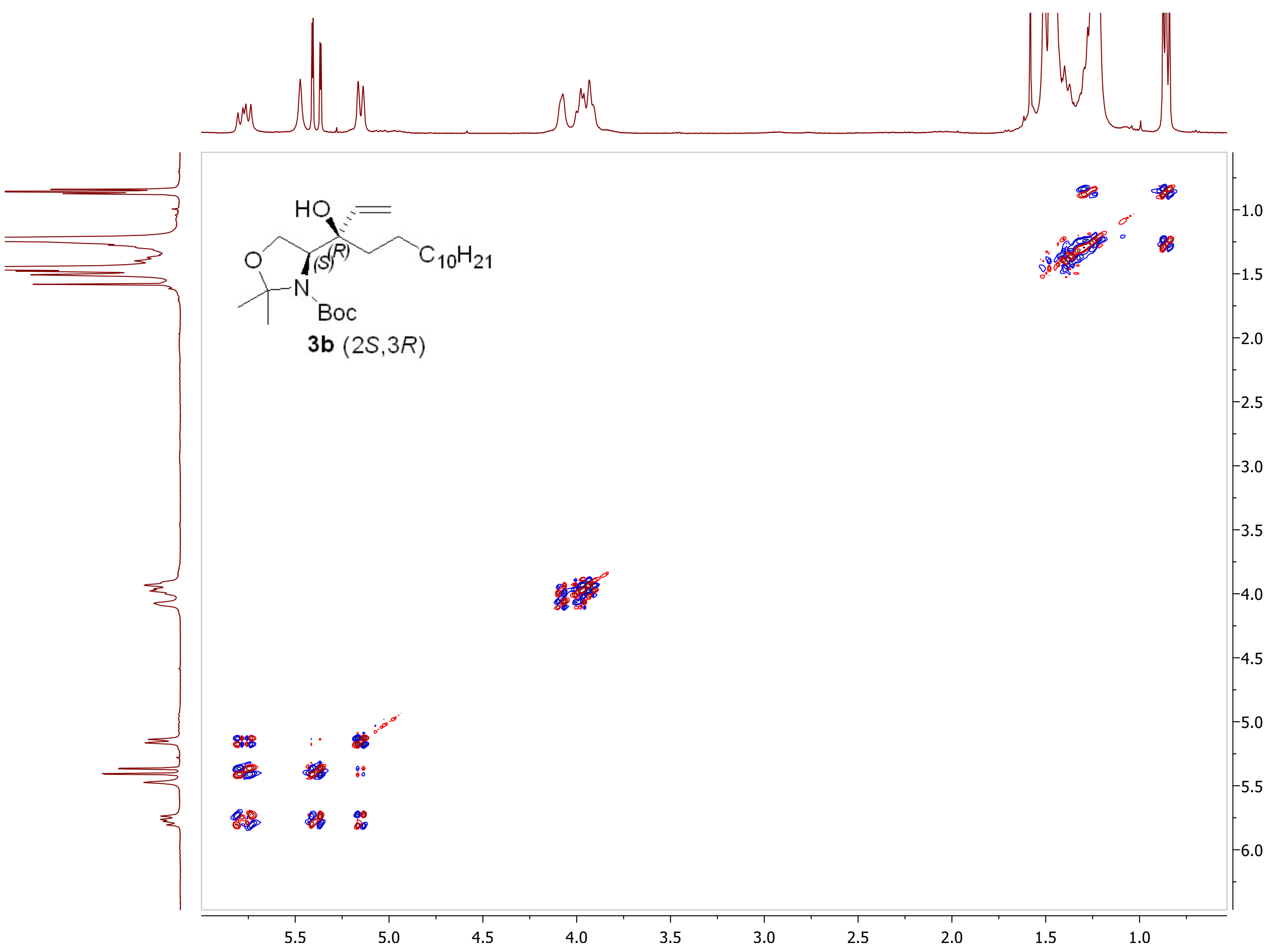




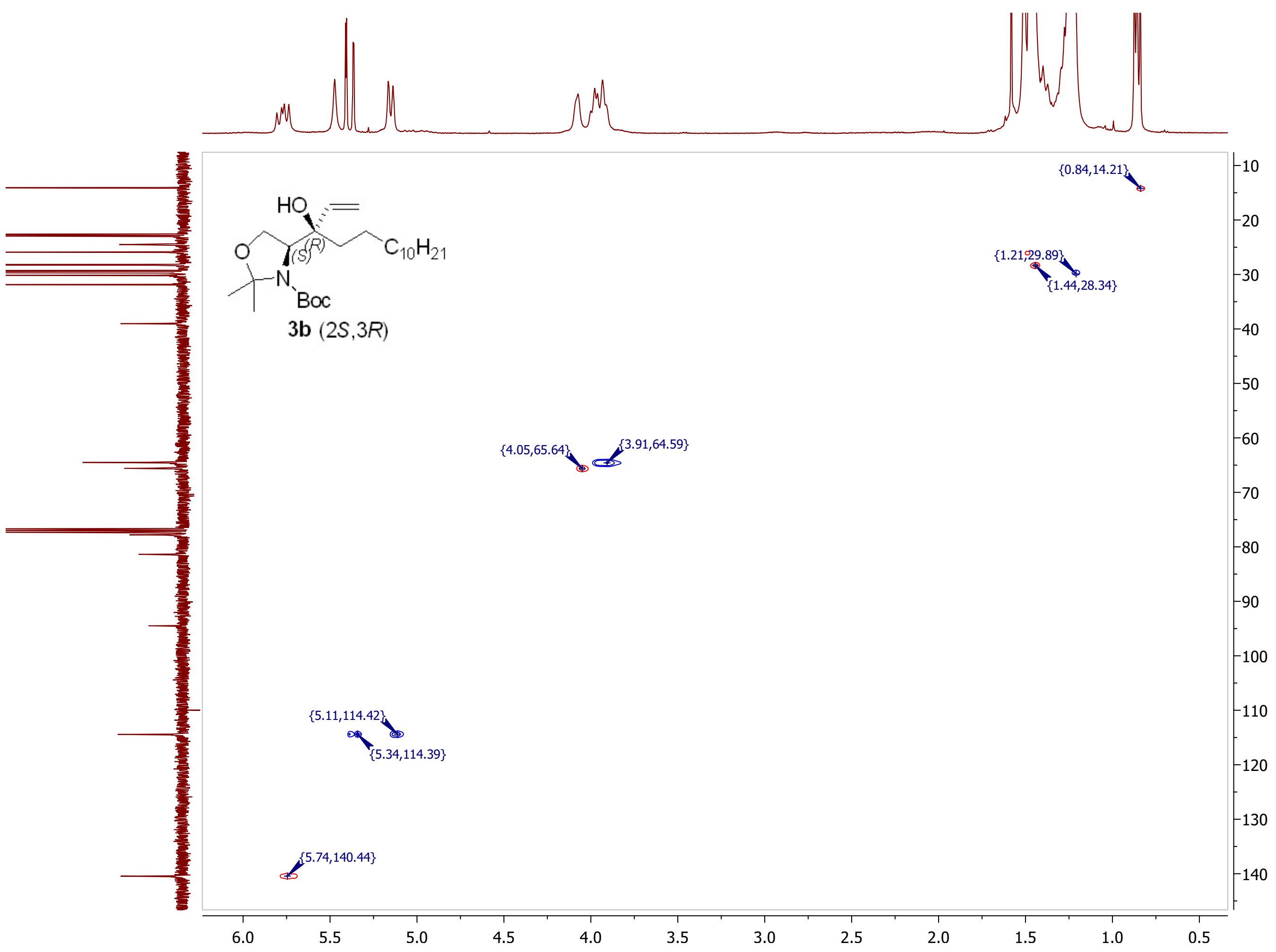




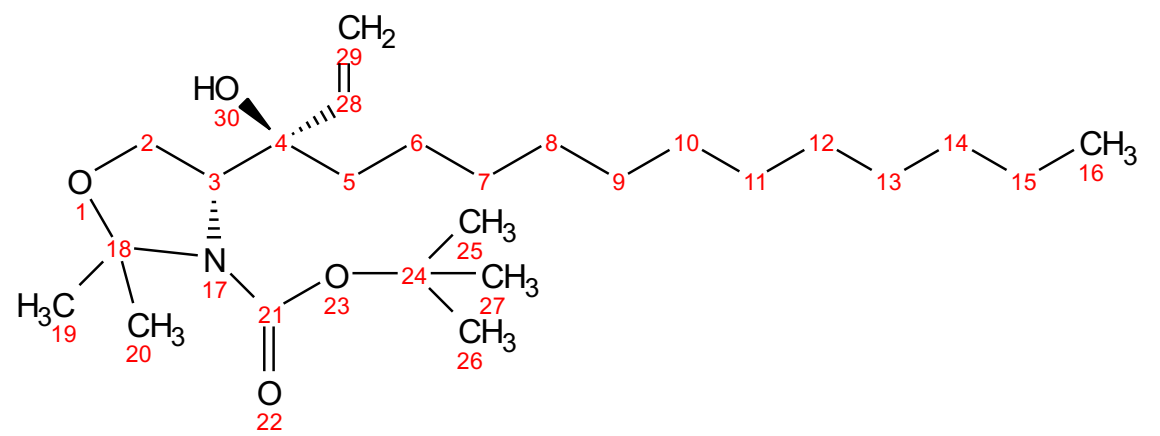

ent-3a $(2 R, 3 R)$

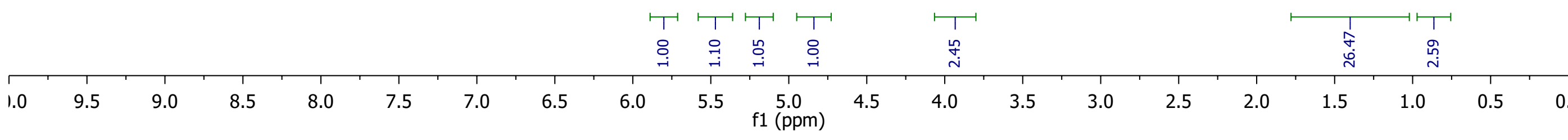




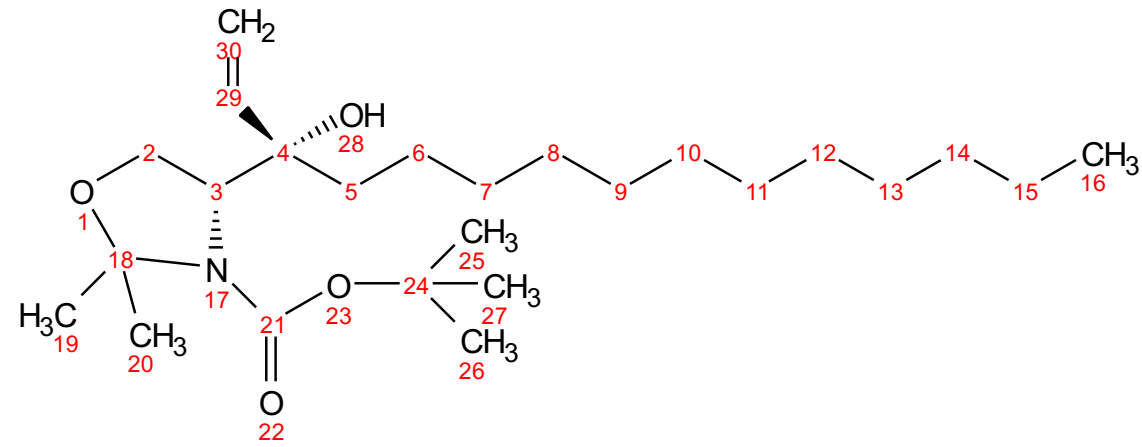

ent-3b $(2 R, 3 S)$

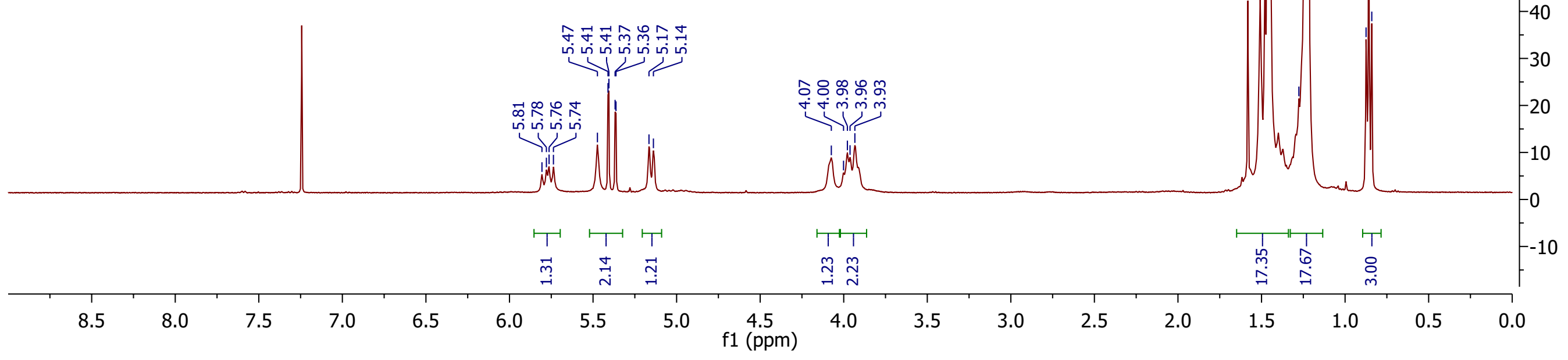




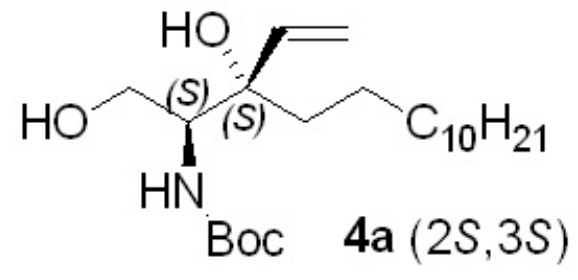




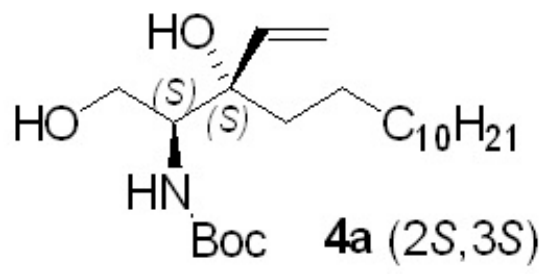




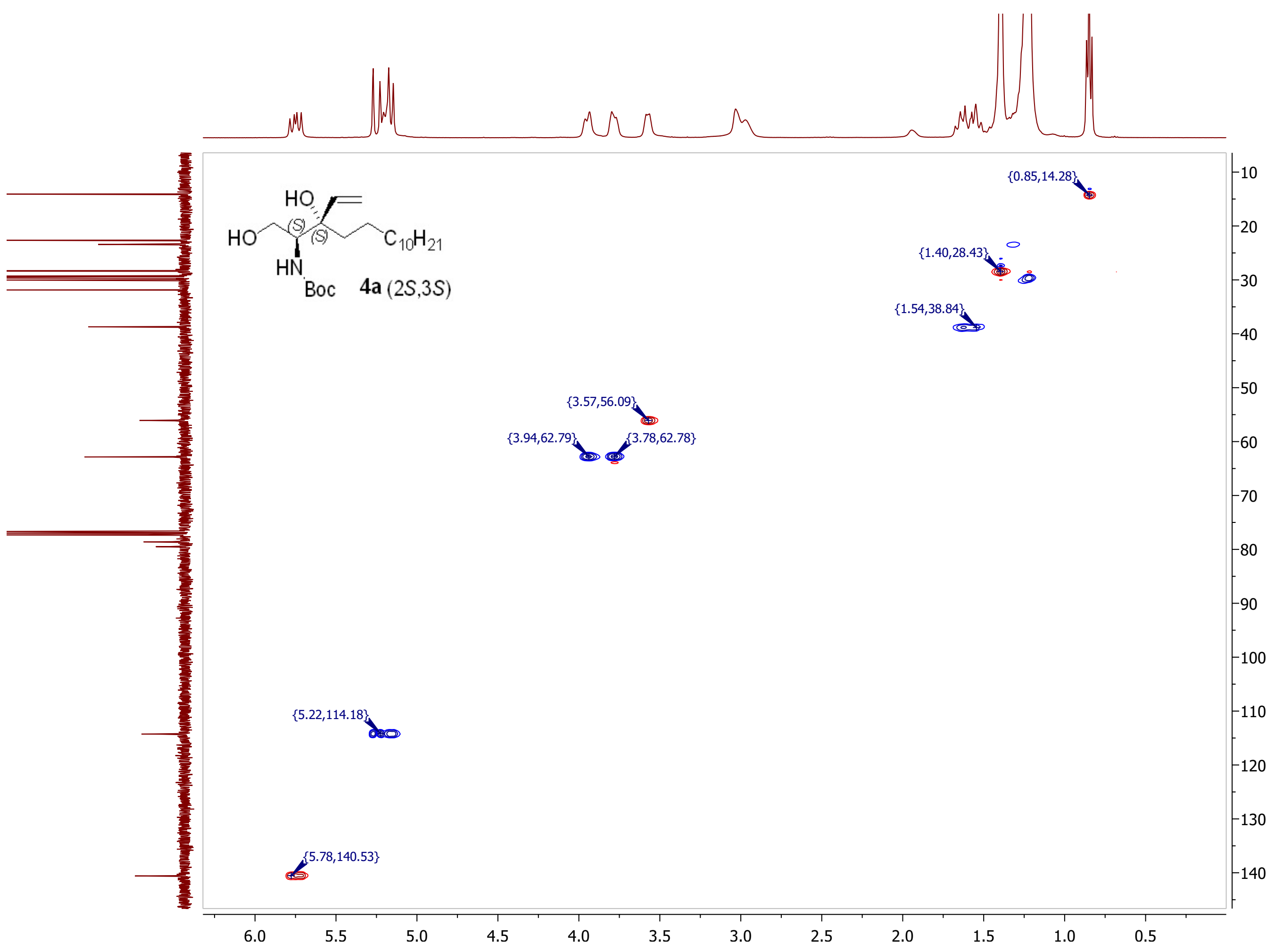




$$
\mathrm{C}_{\mathrm{Boc}} \quad 4 \mathrm{~b}(2 \mathrm{H}, 3 \mathrm{H})
$$

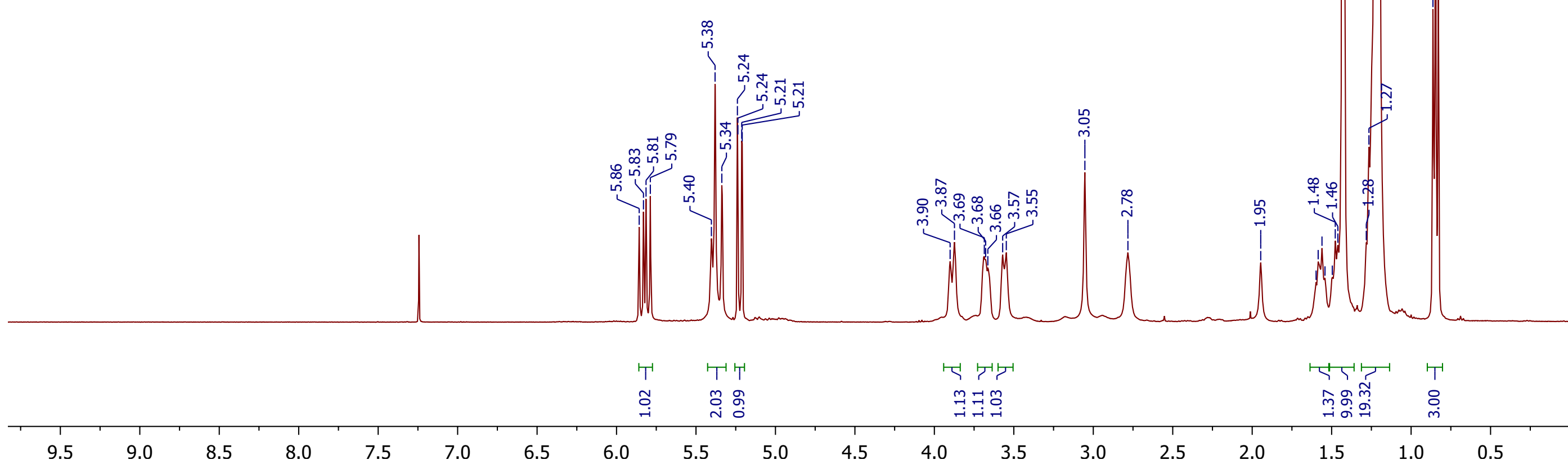




$$
\text { Boc } \quad \mathbf{4 b}(2 \mathrm{~S}, 3 R)
$$

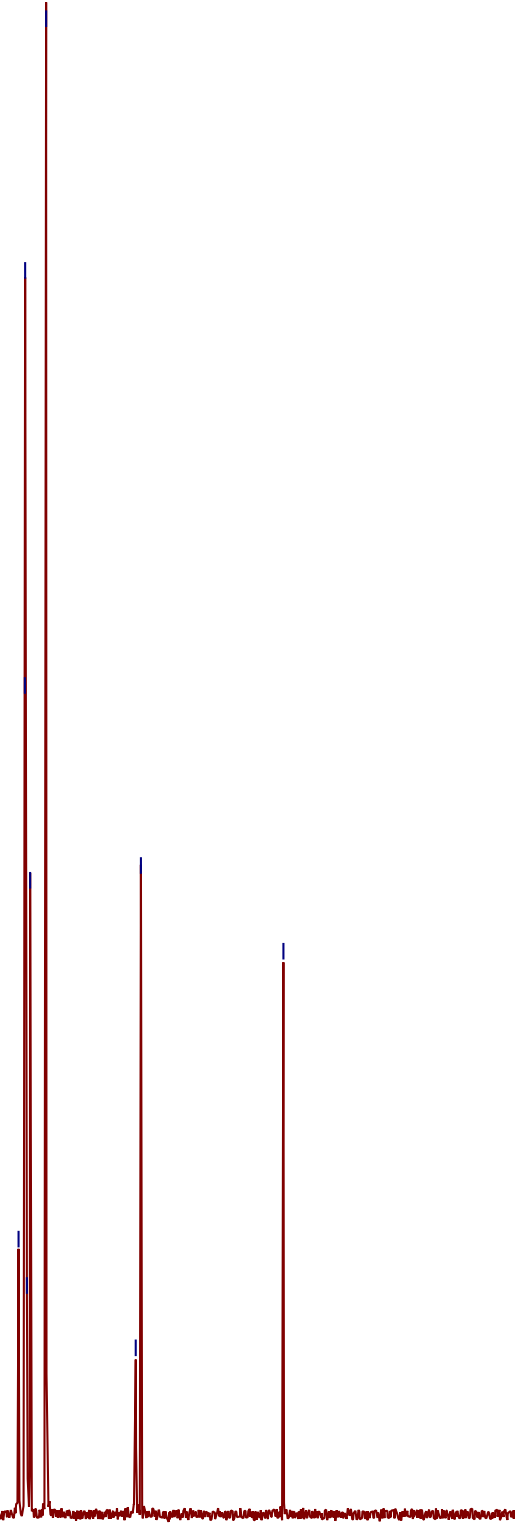




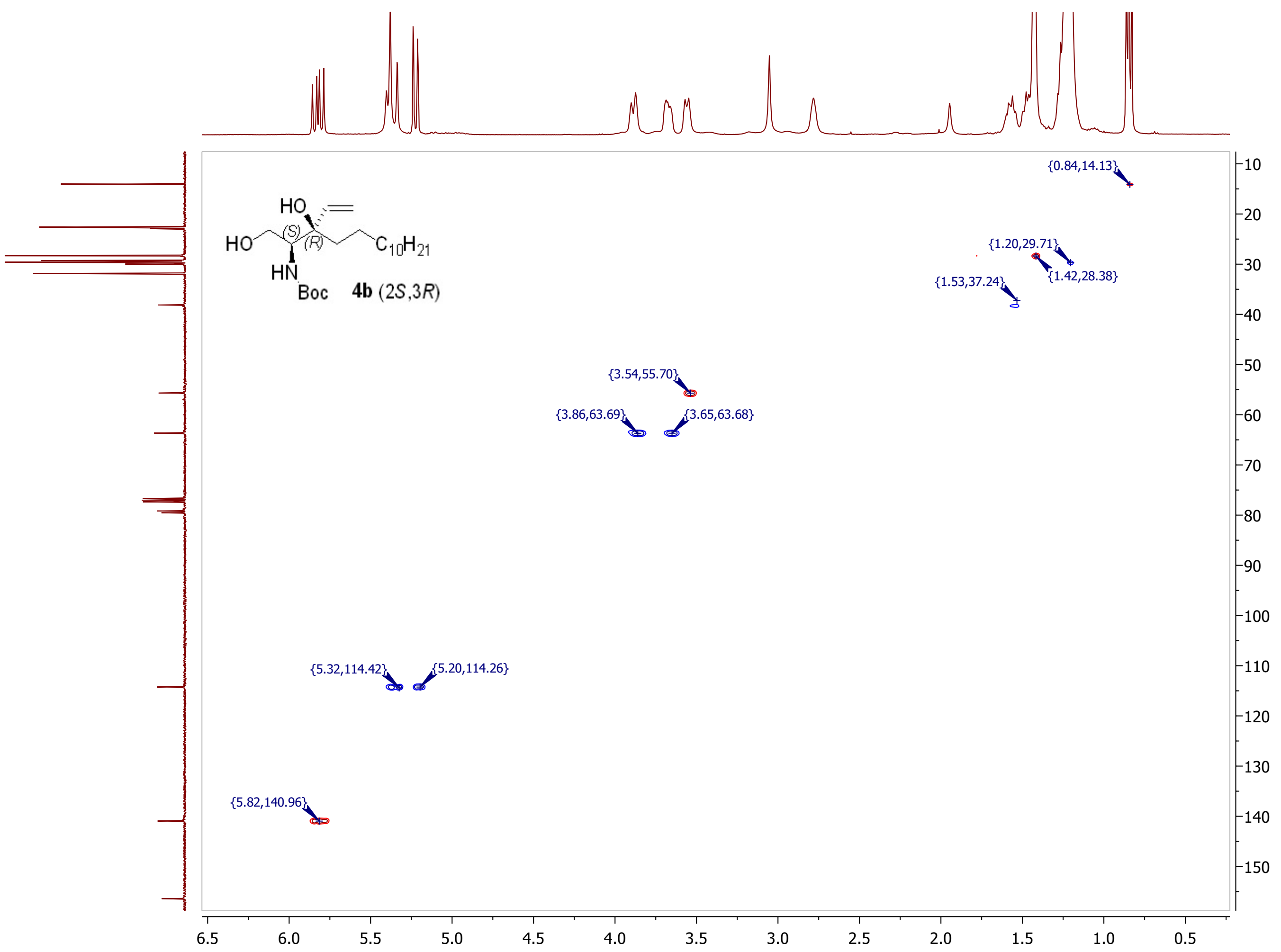




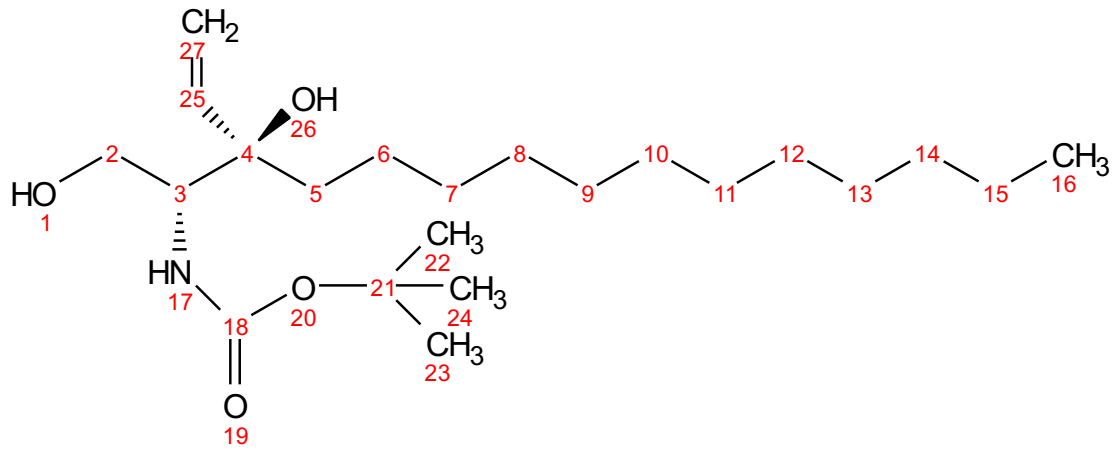

ent-4a (2R,3R)

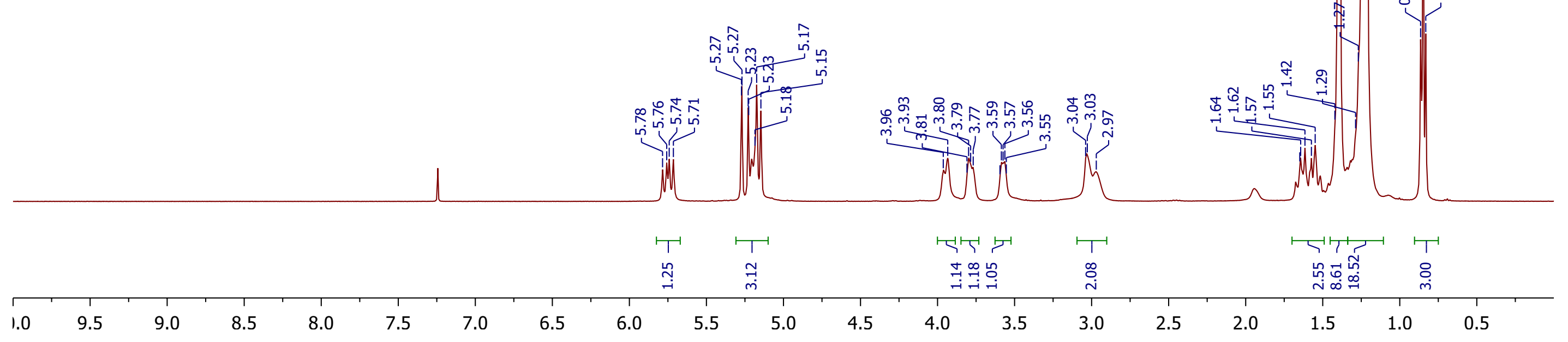




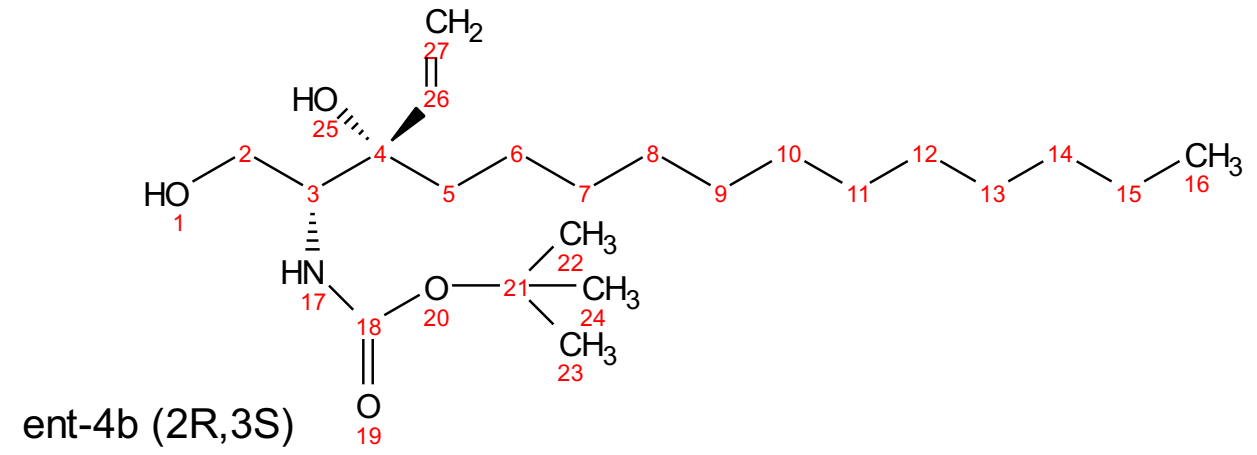

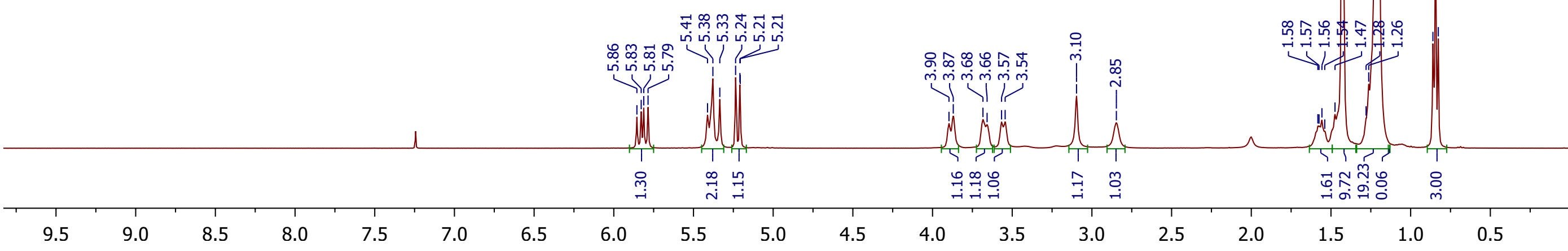




$$
\text { 5a }(2 S, 3 S)
$$

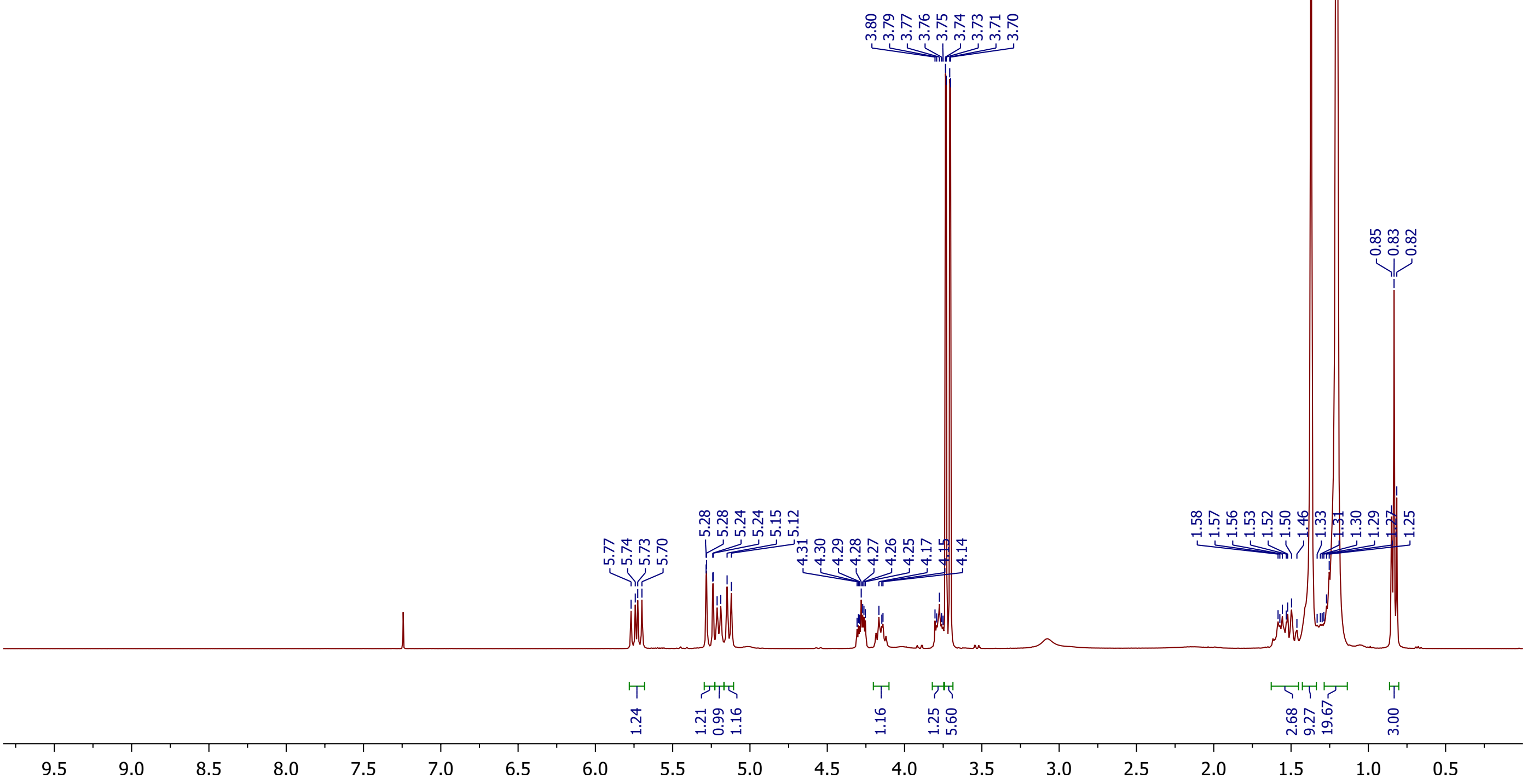




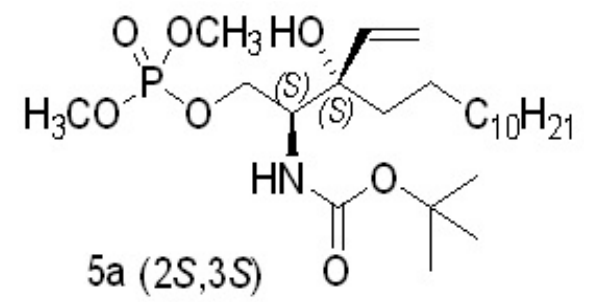




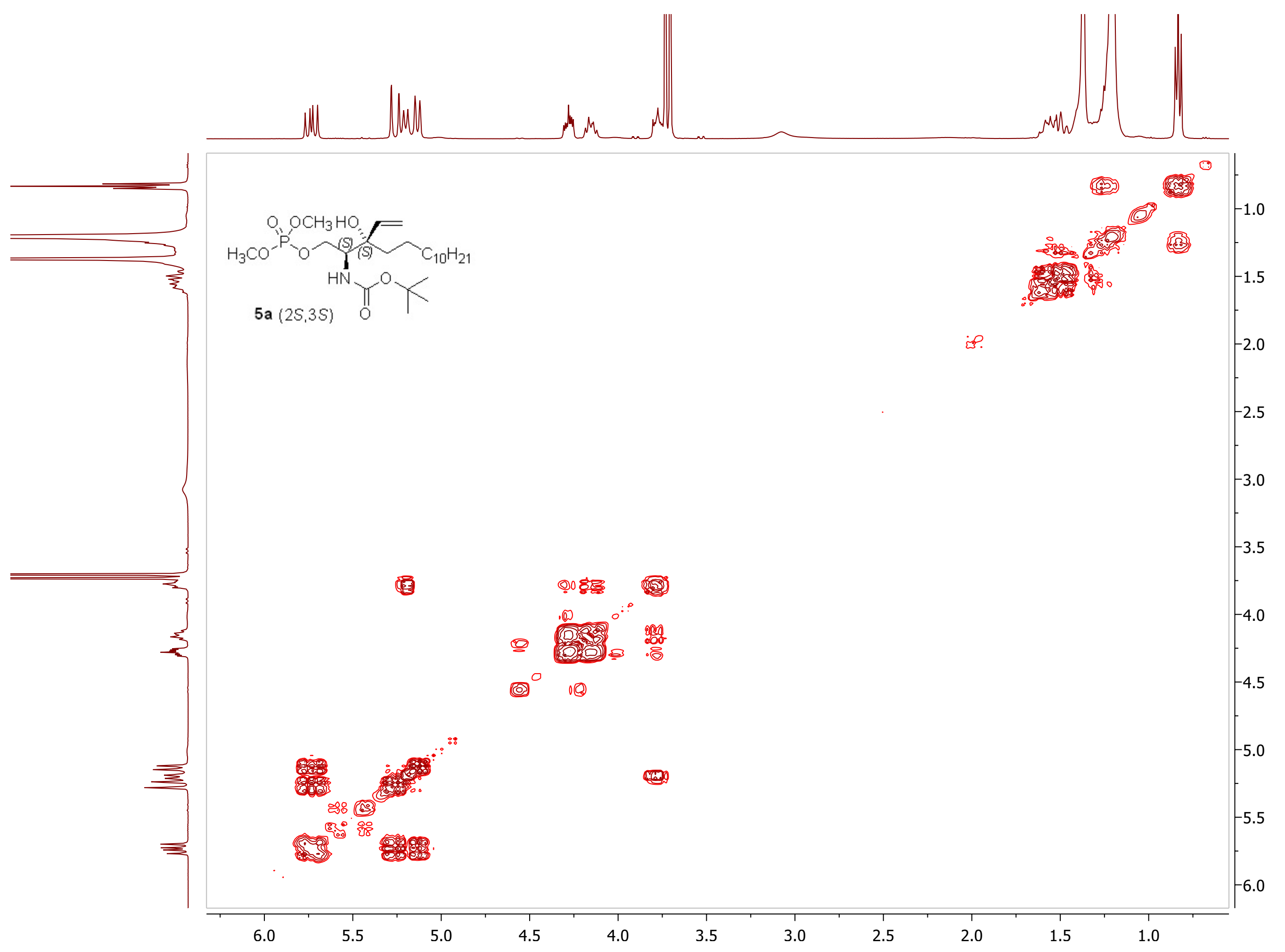



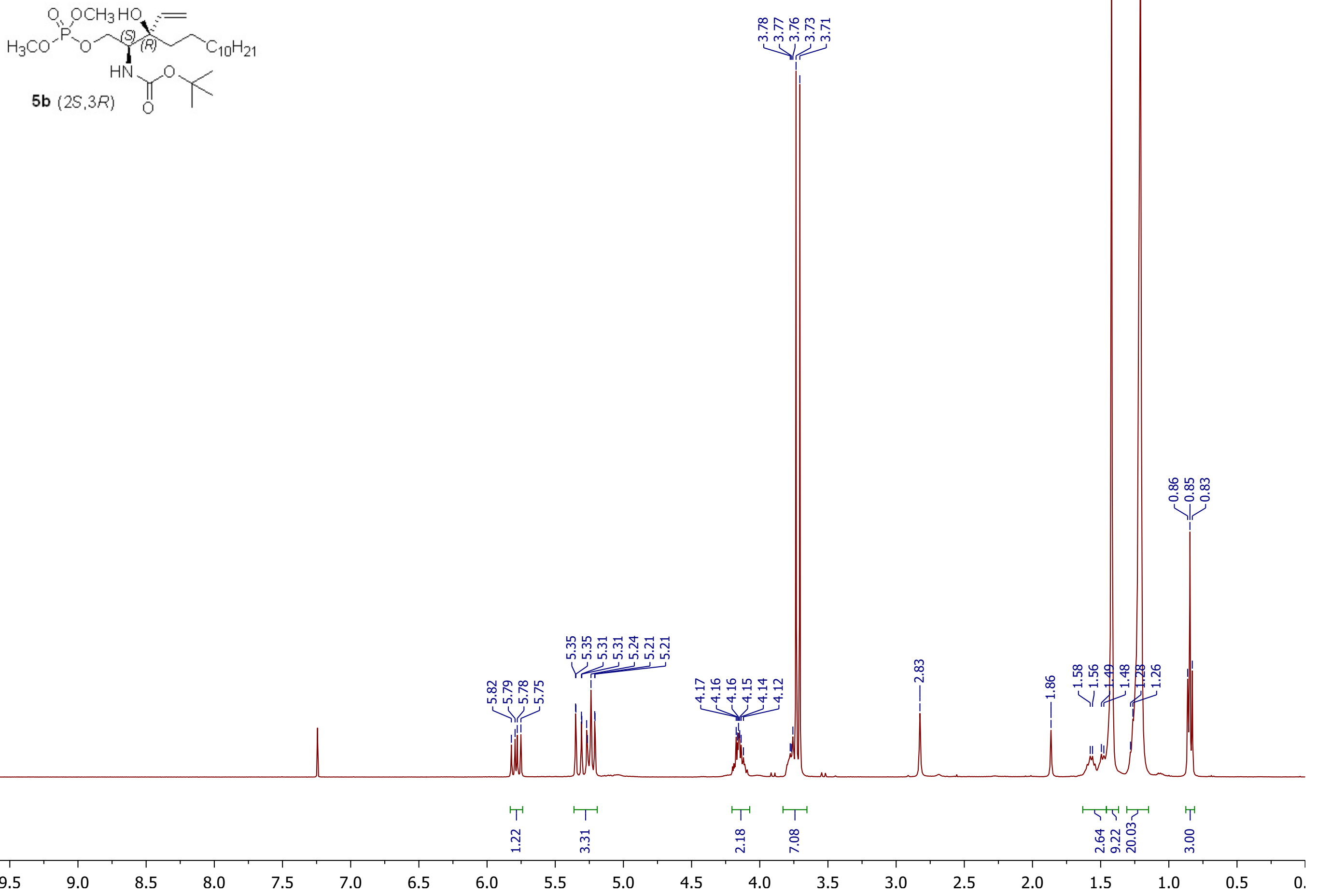


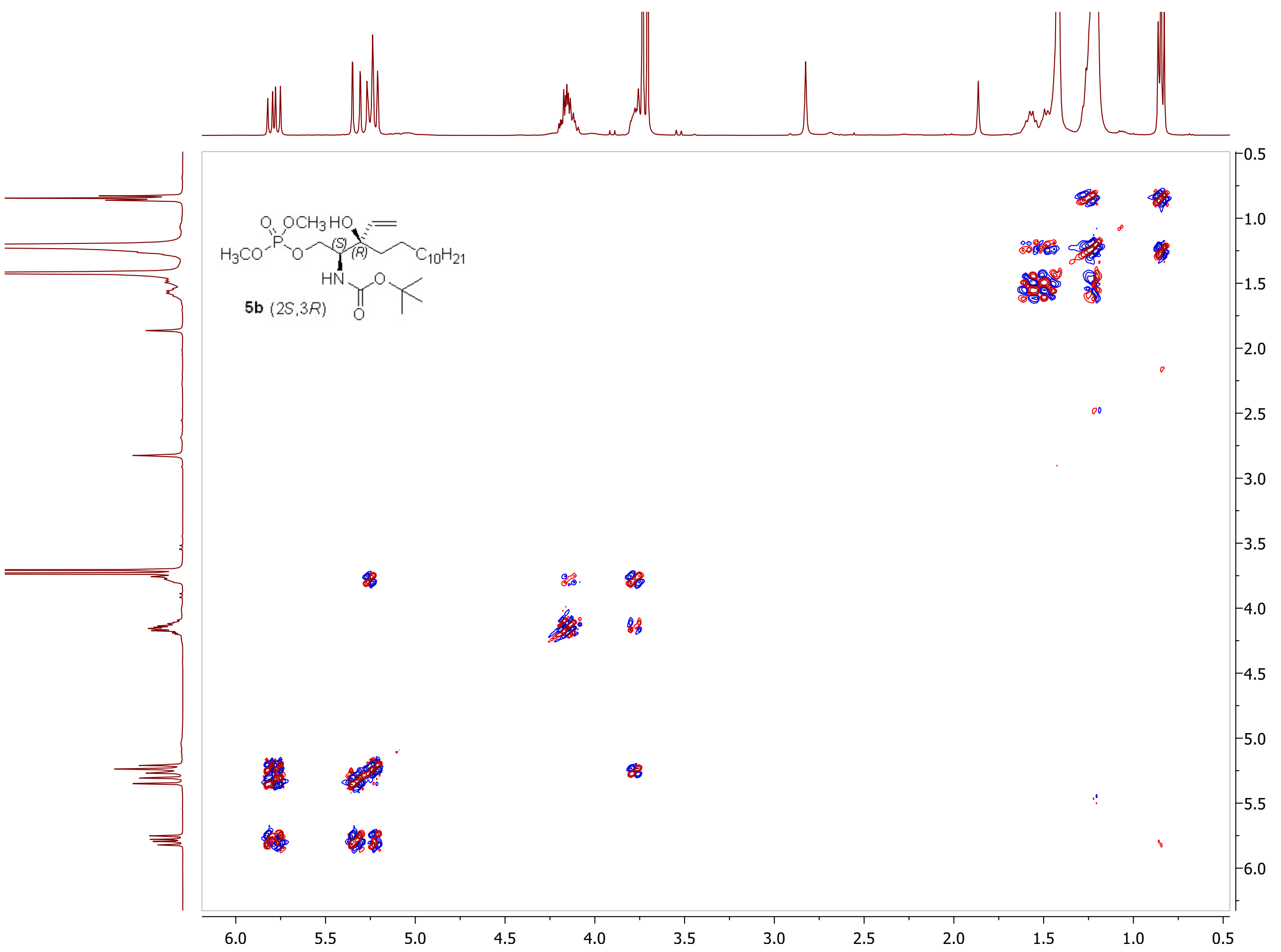




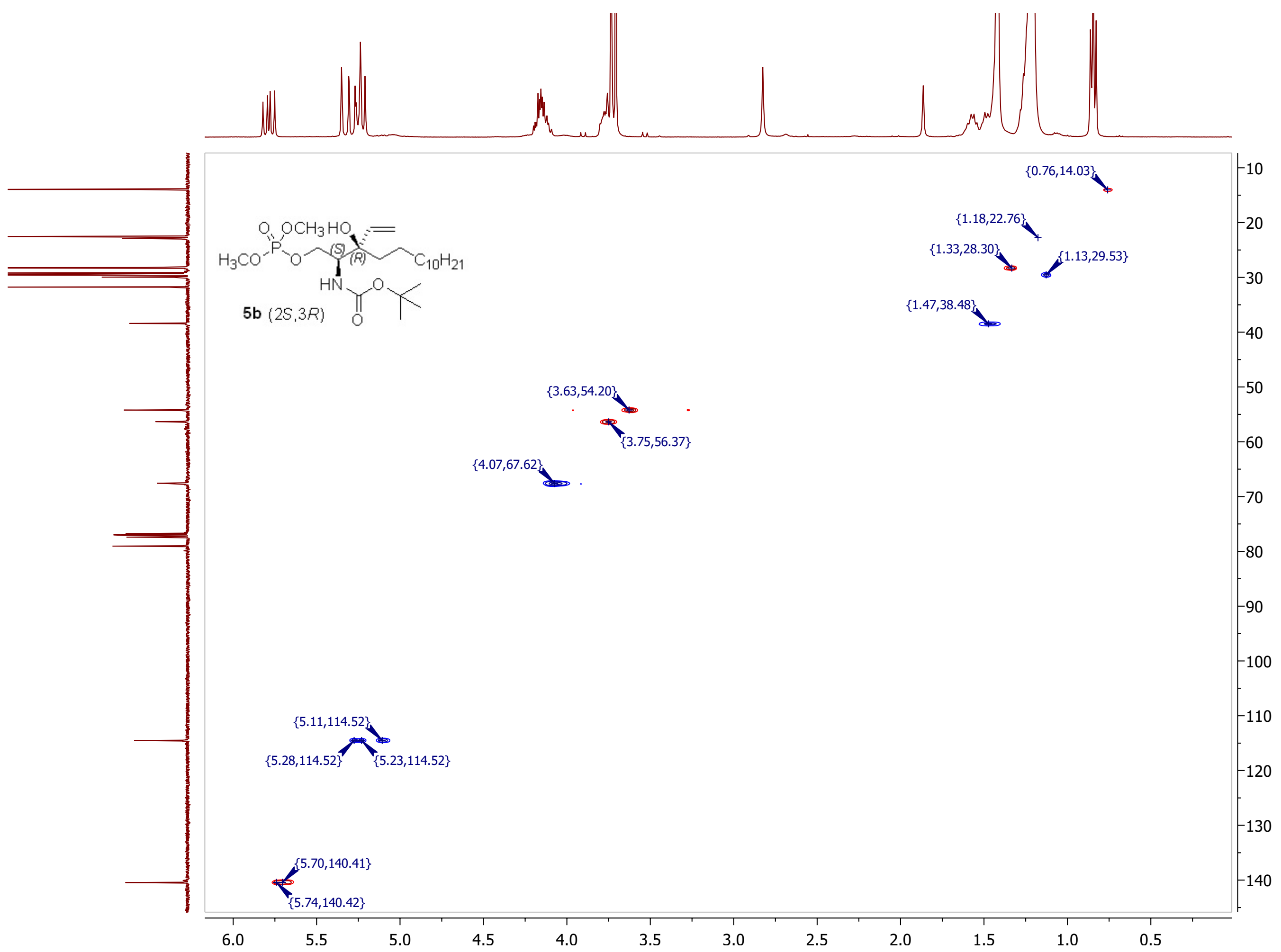




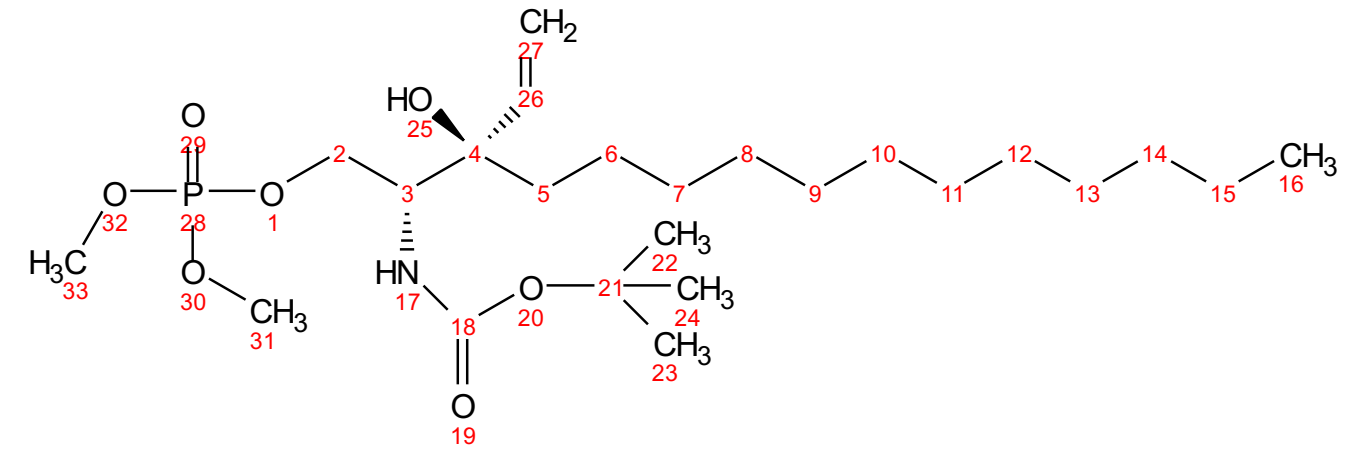

ent-5a $(R, R)$

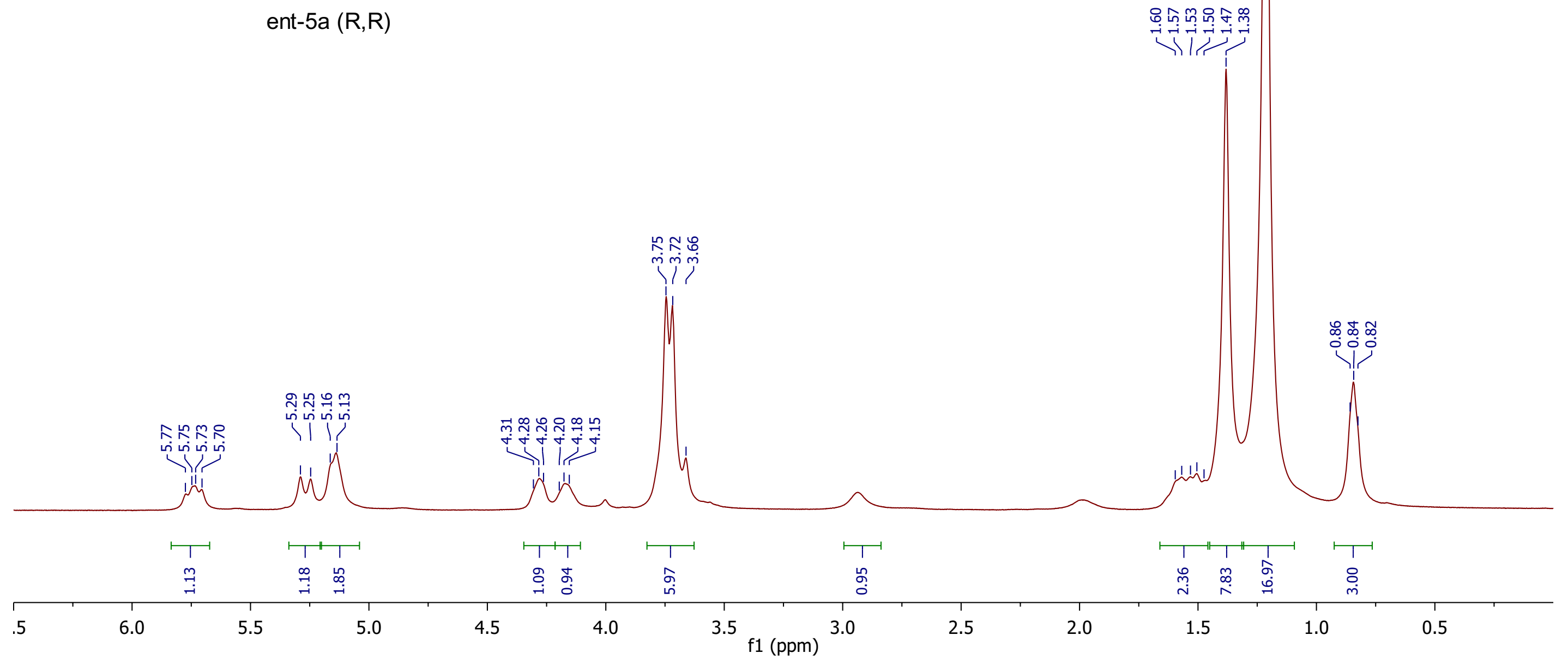




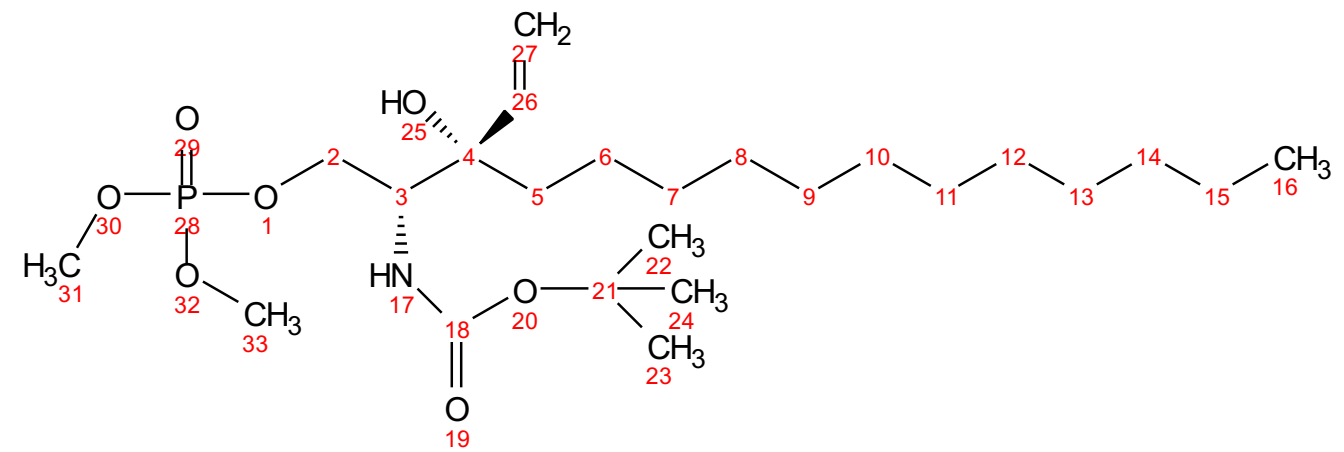

ent-5b $(2 R, 3 S)$

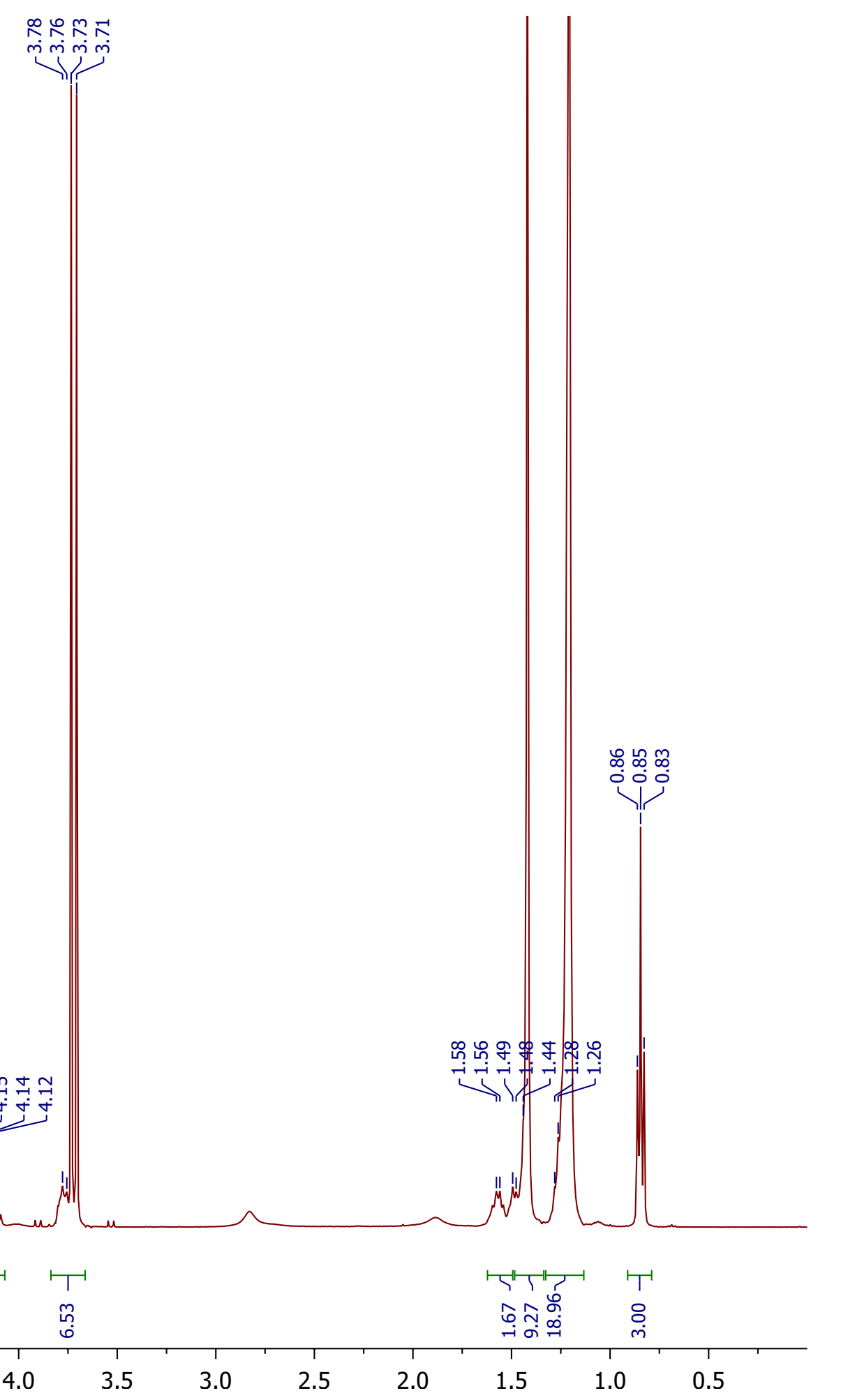



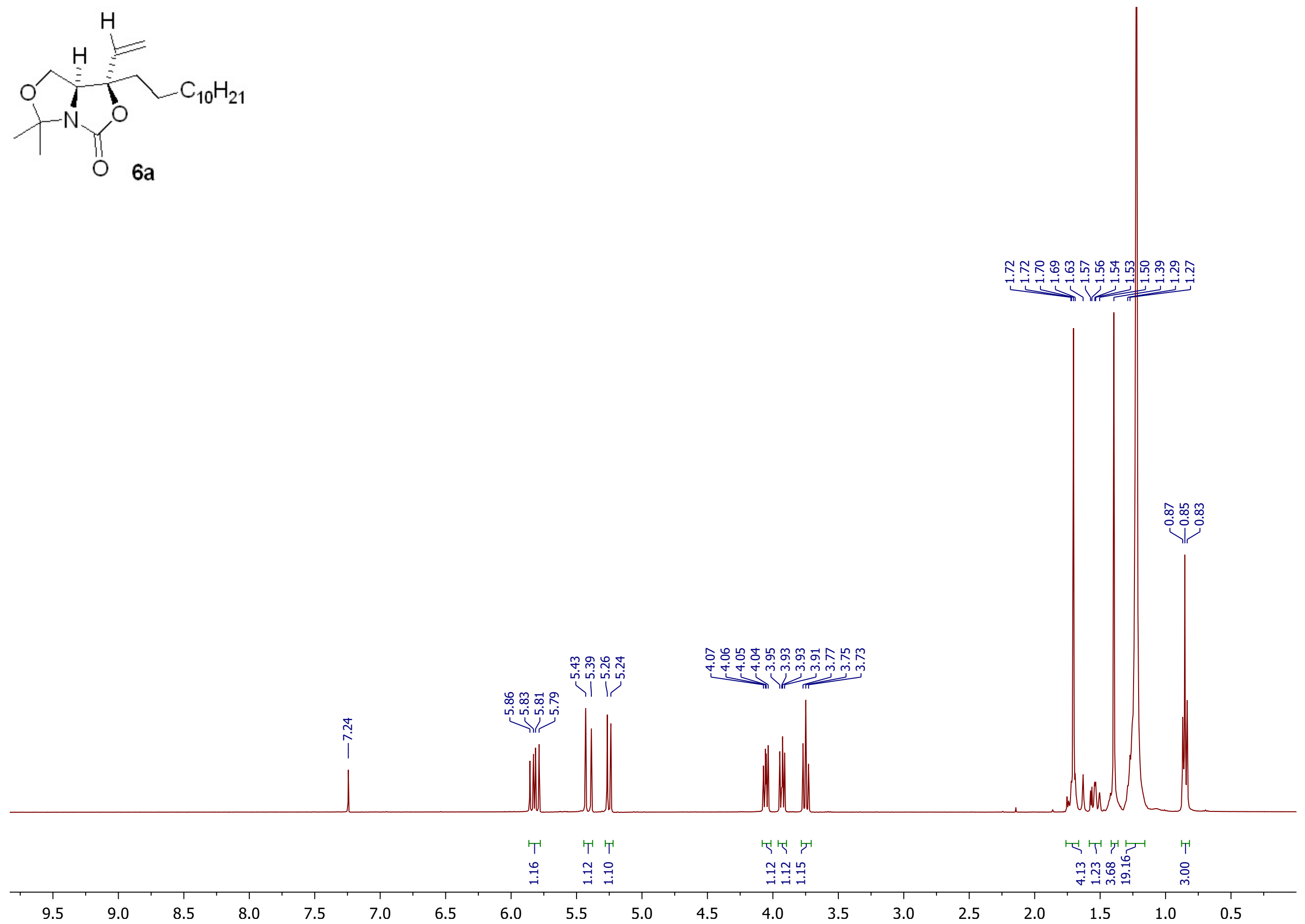


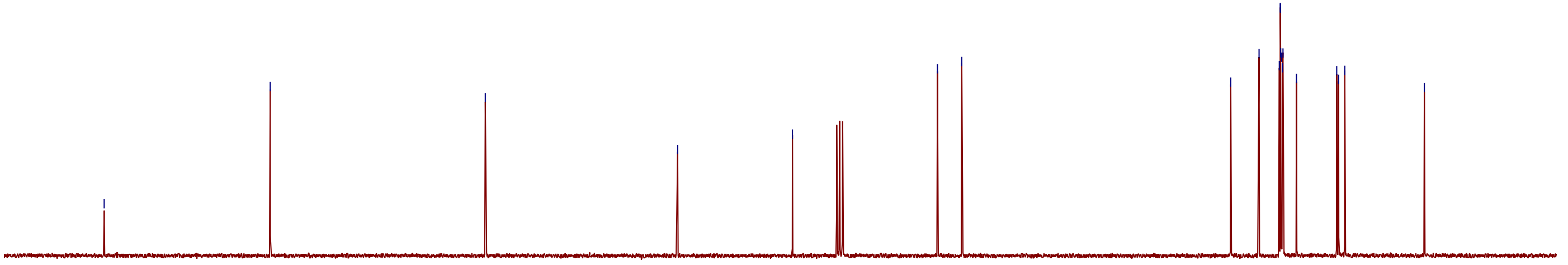




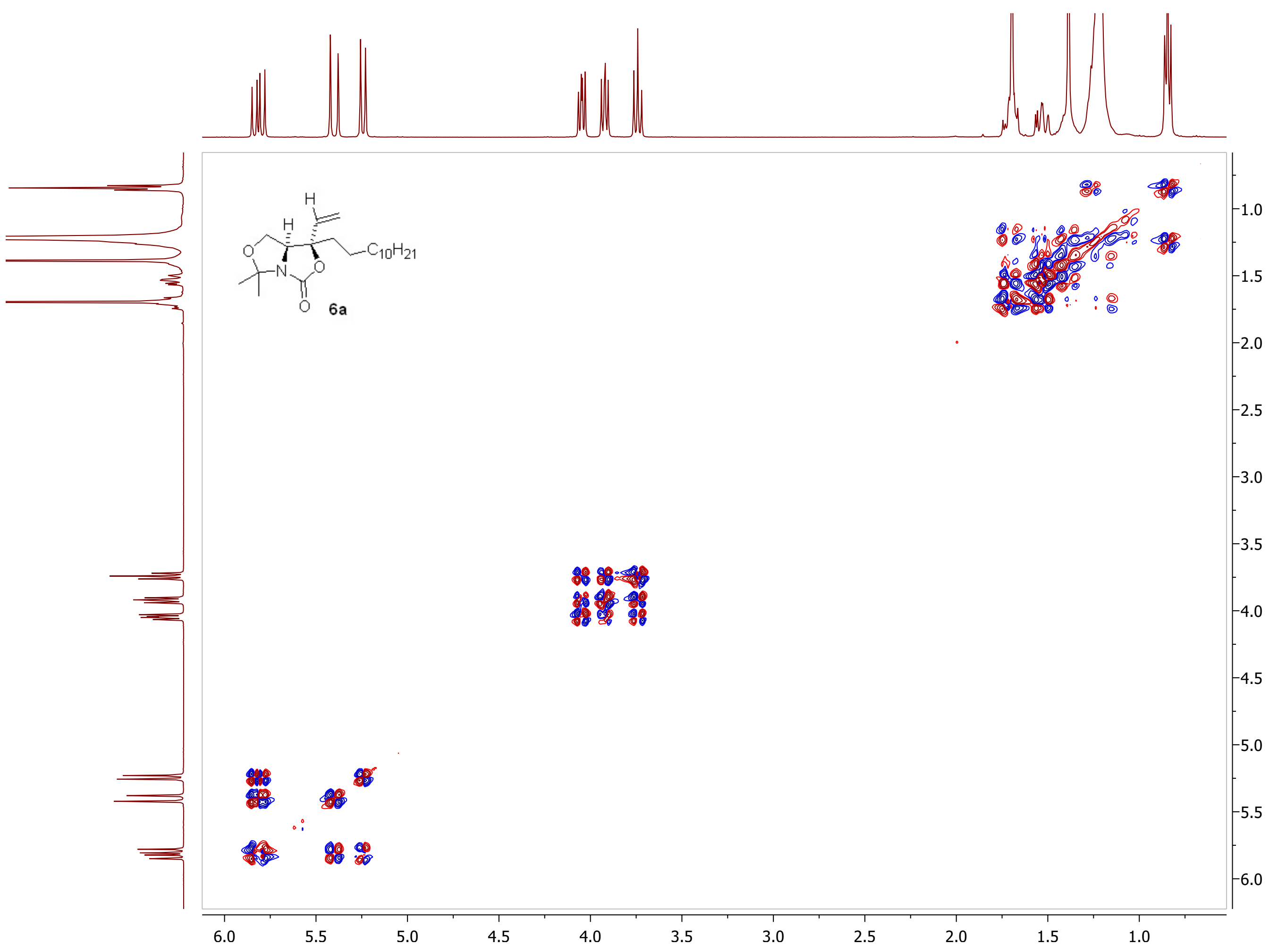




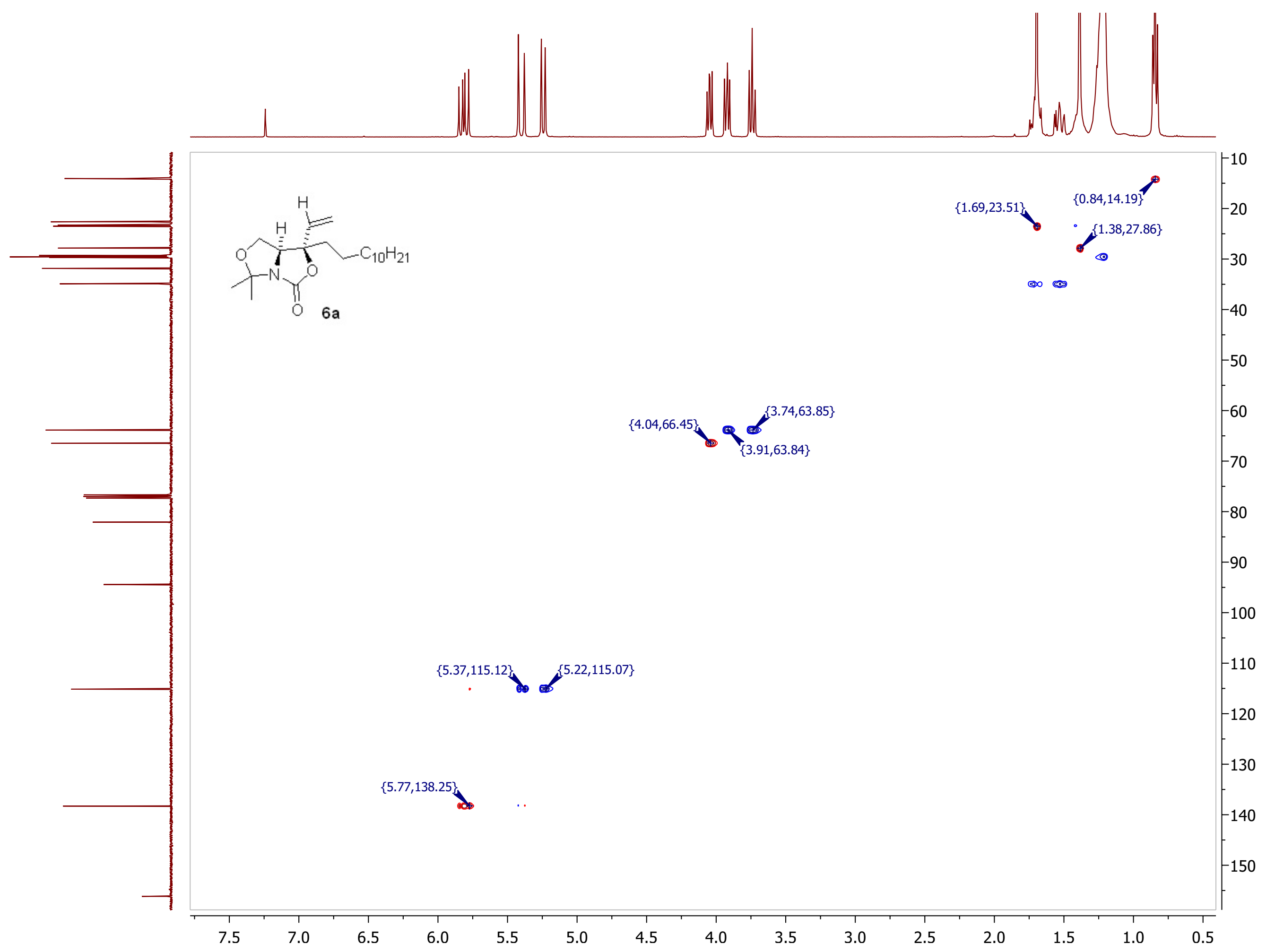




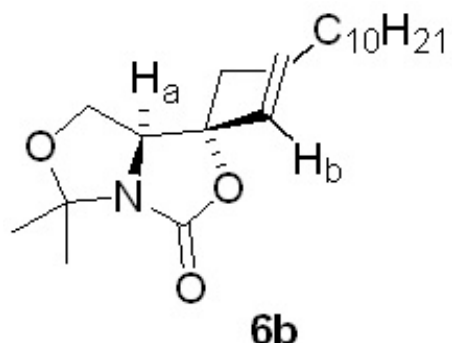

$6 b$

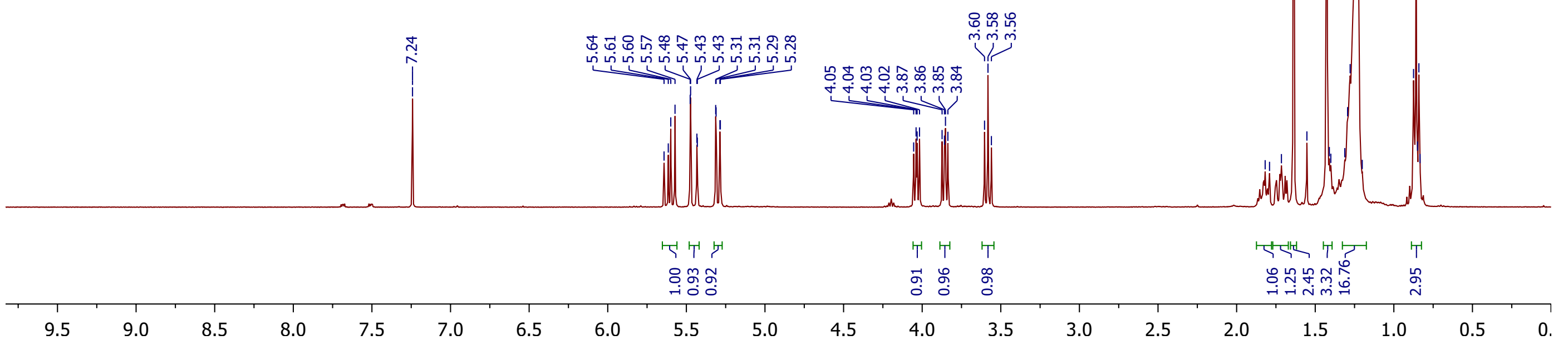



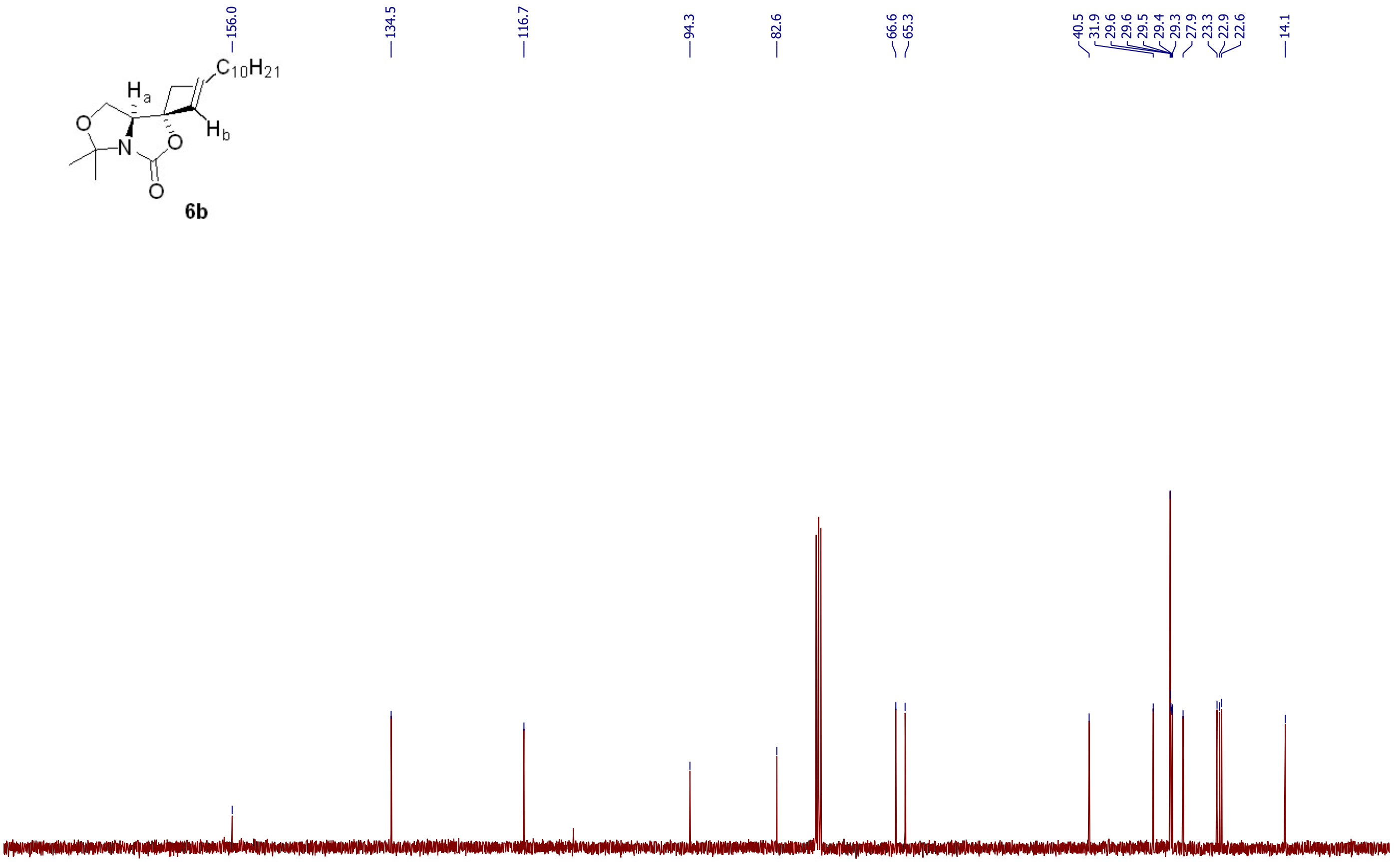


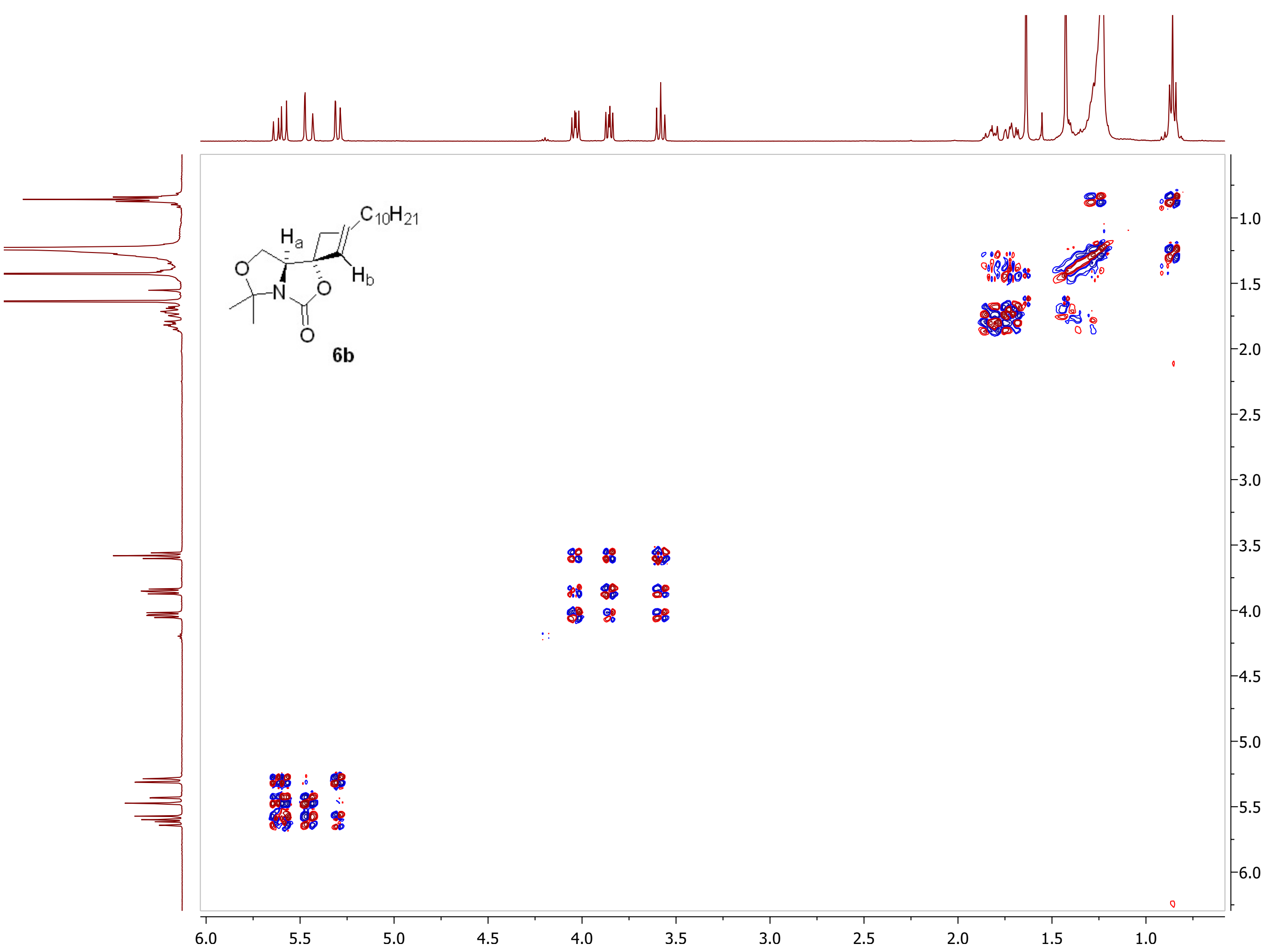




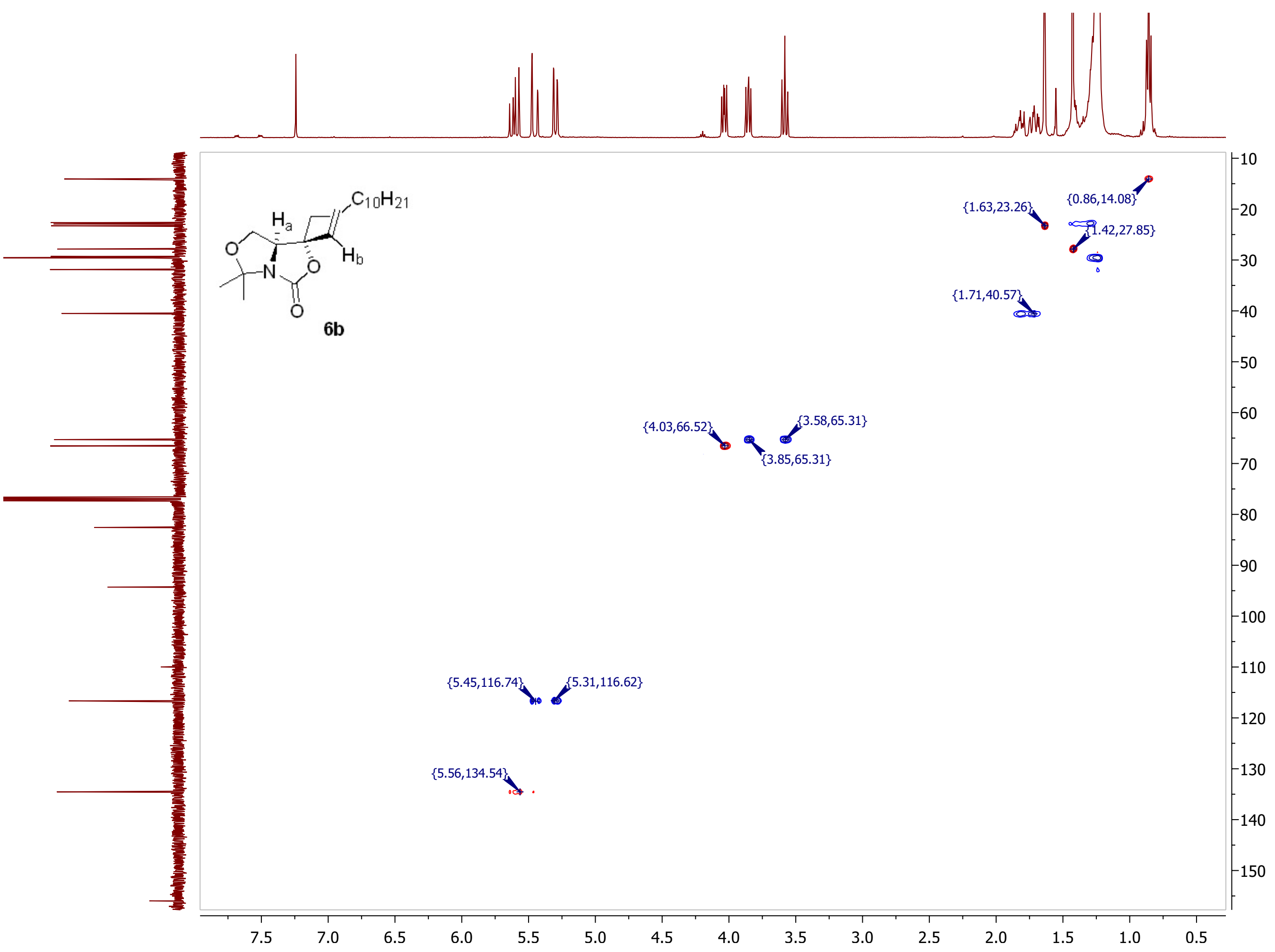




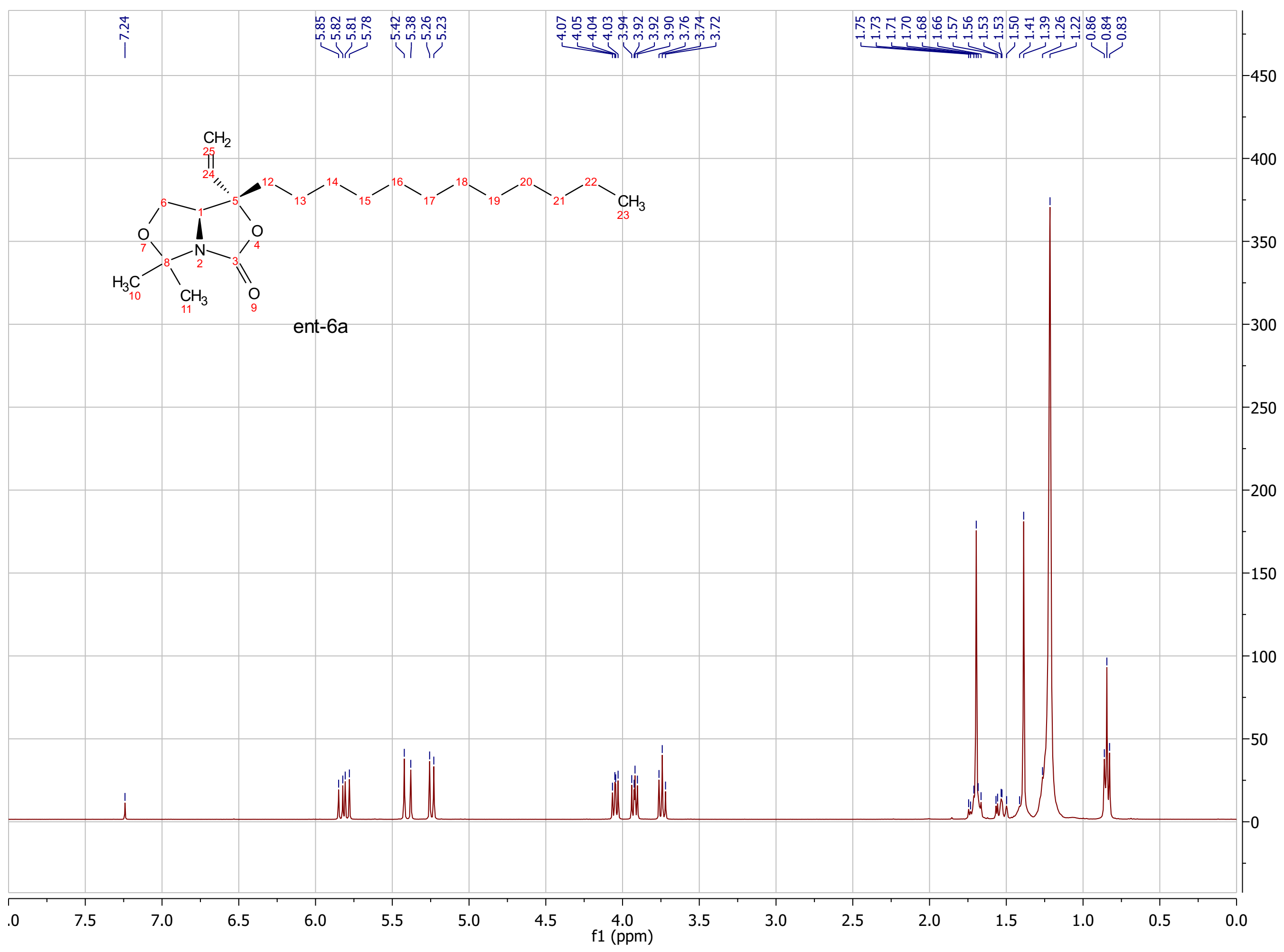




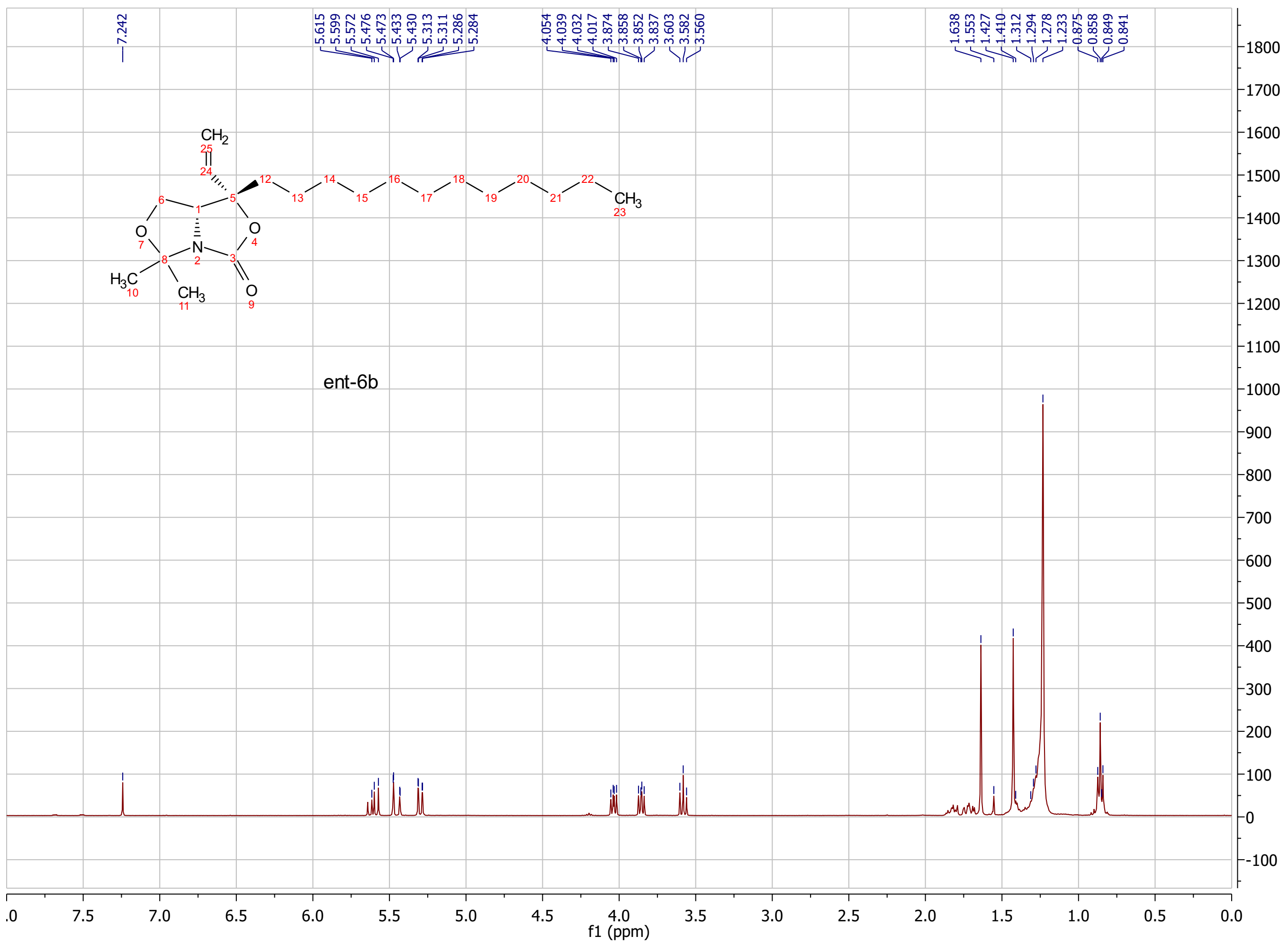



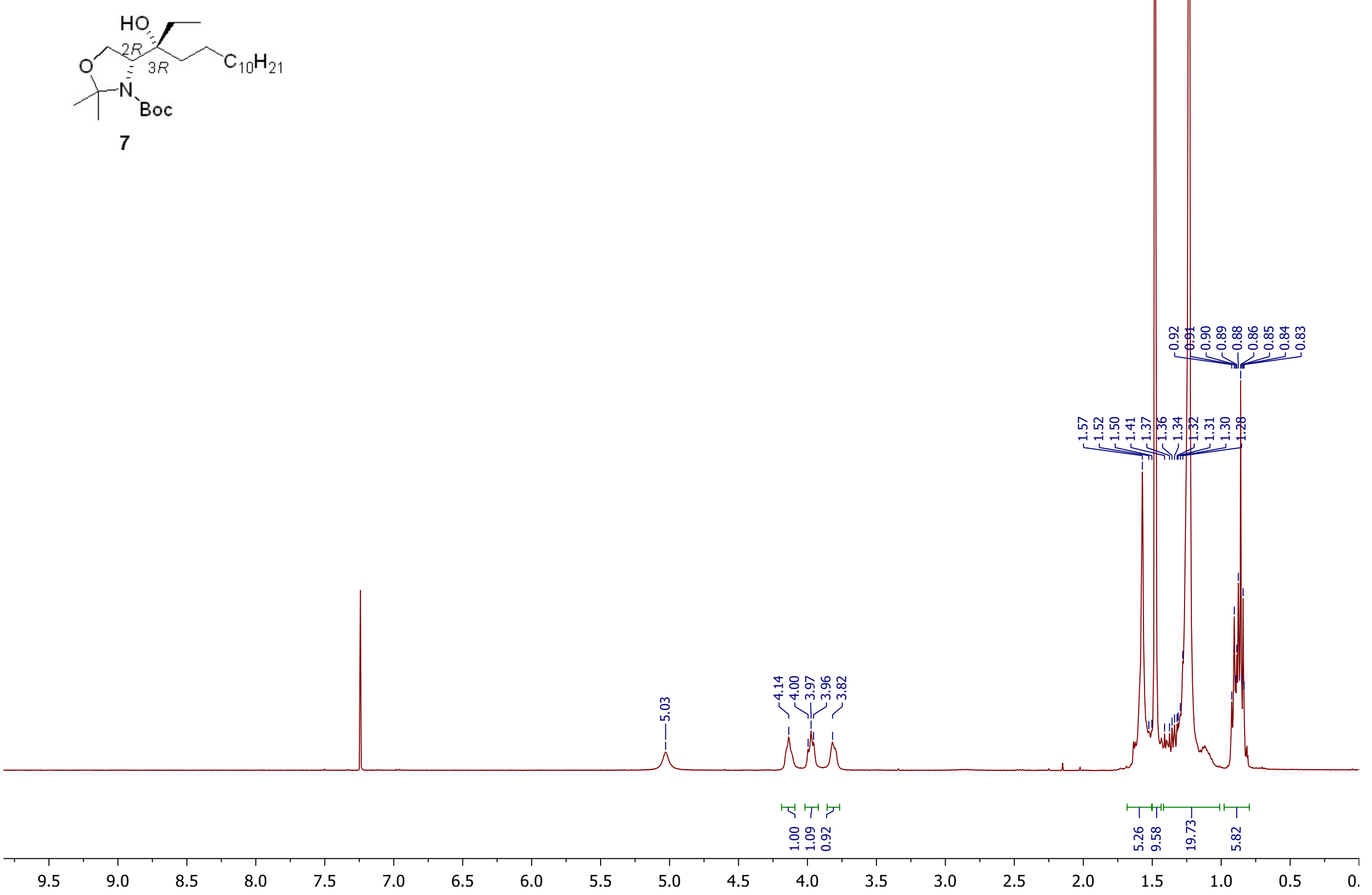


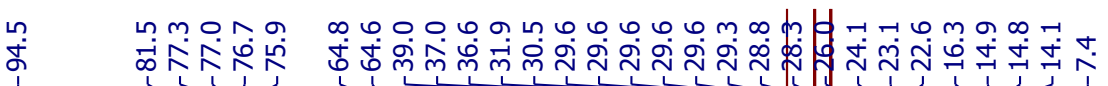
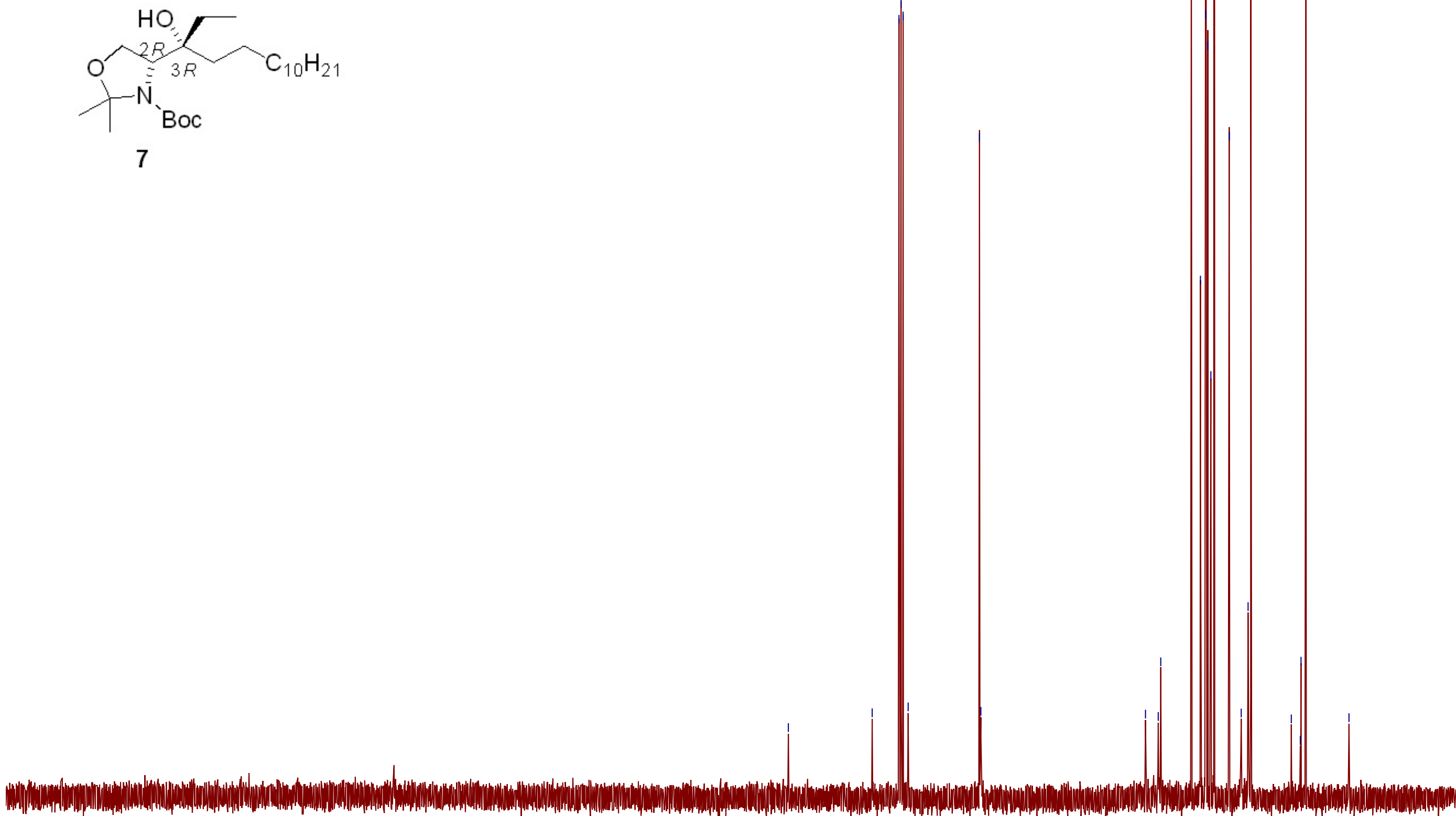

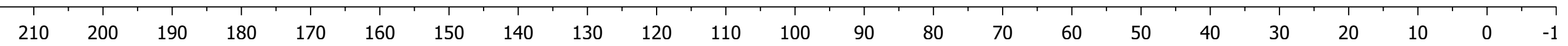




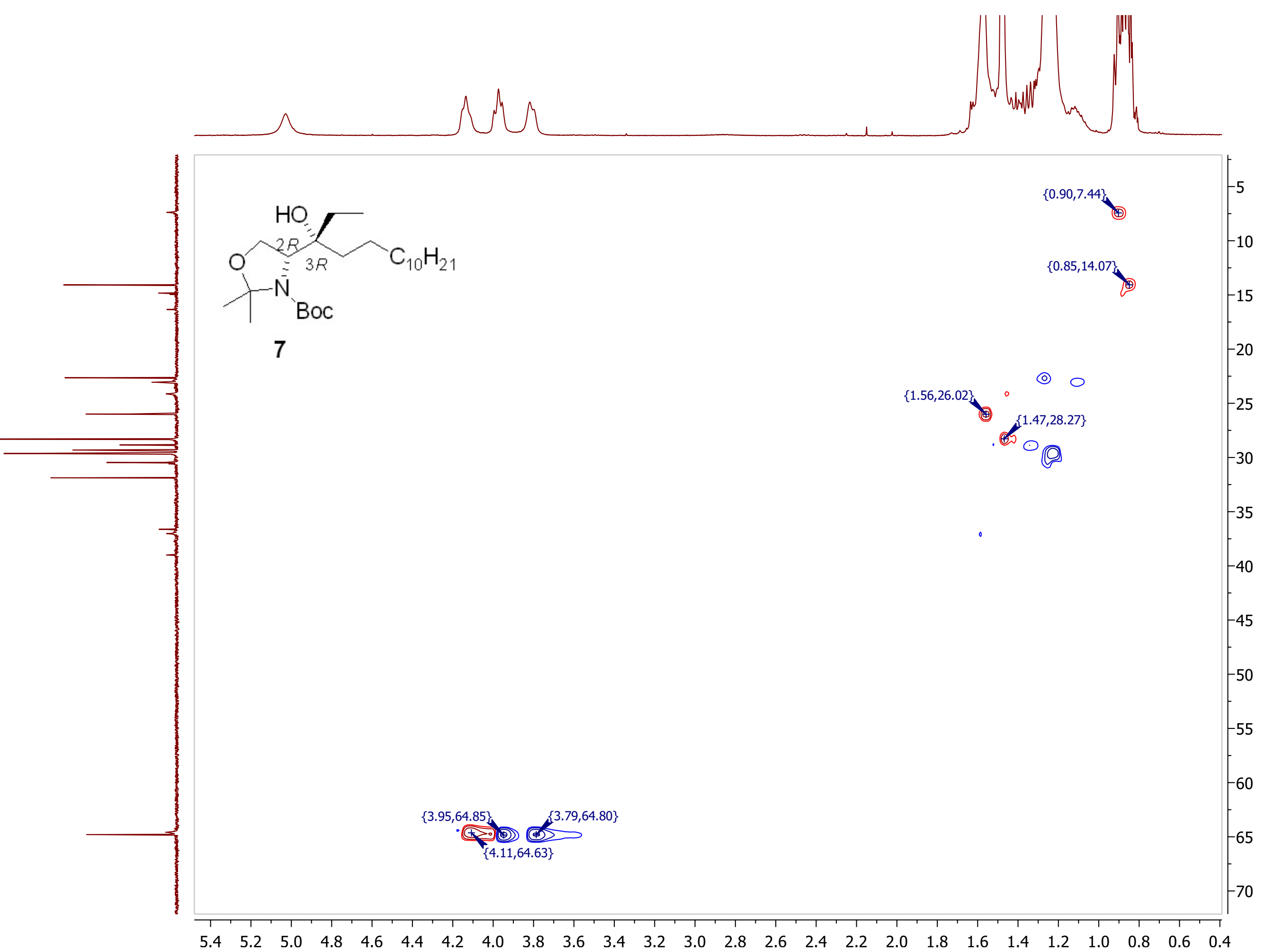



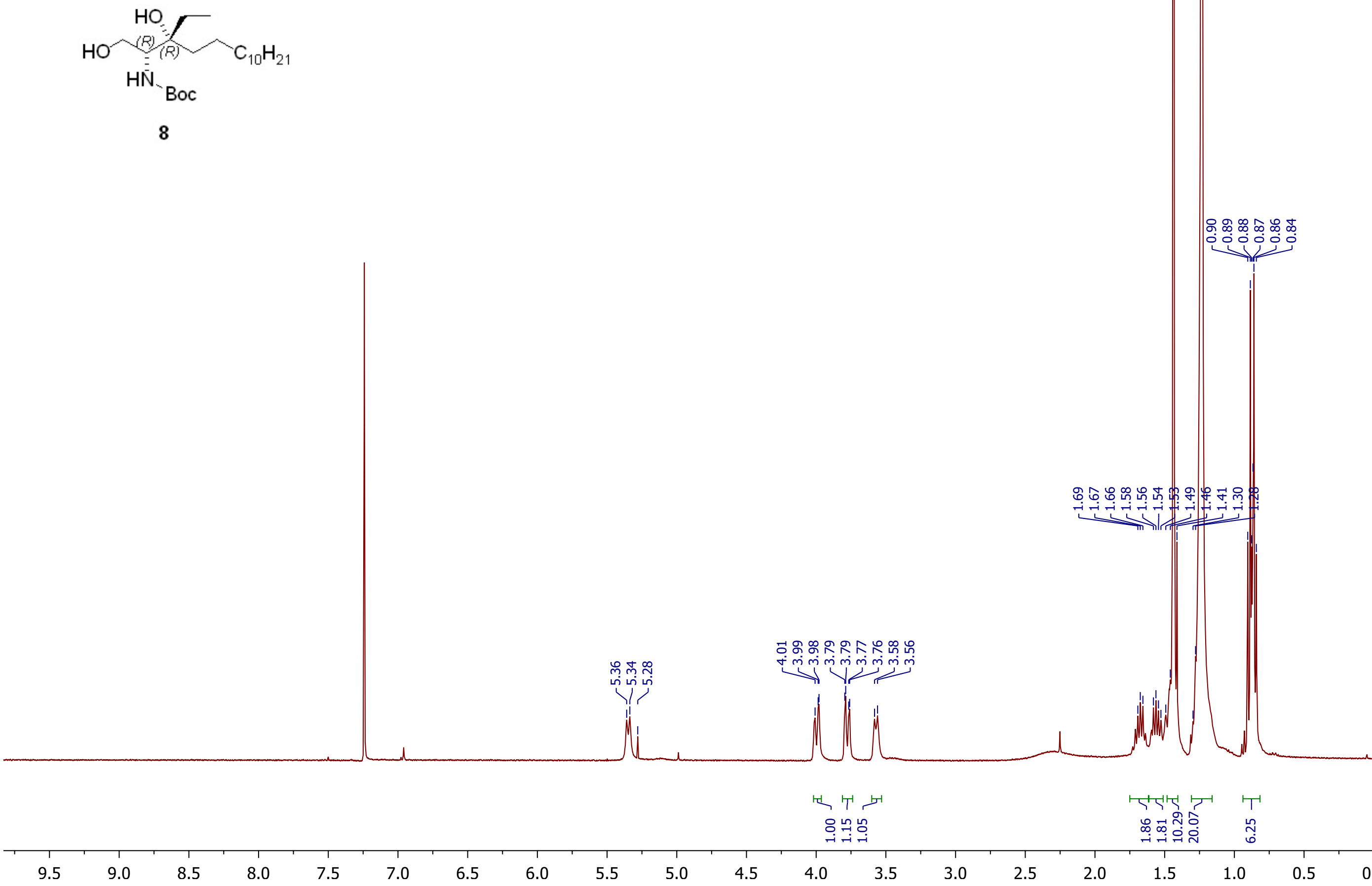
$\mathrm{C}_{10} \mathrm{H}_{21}$

8

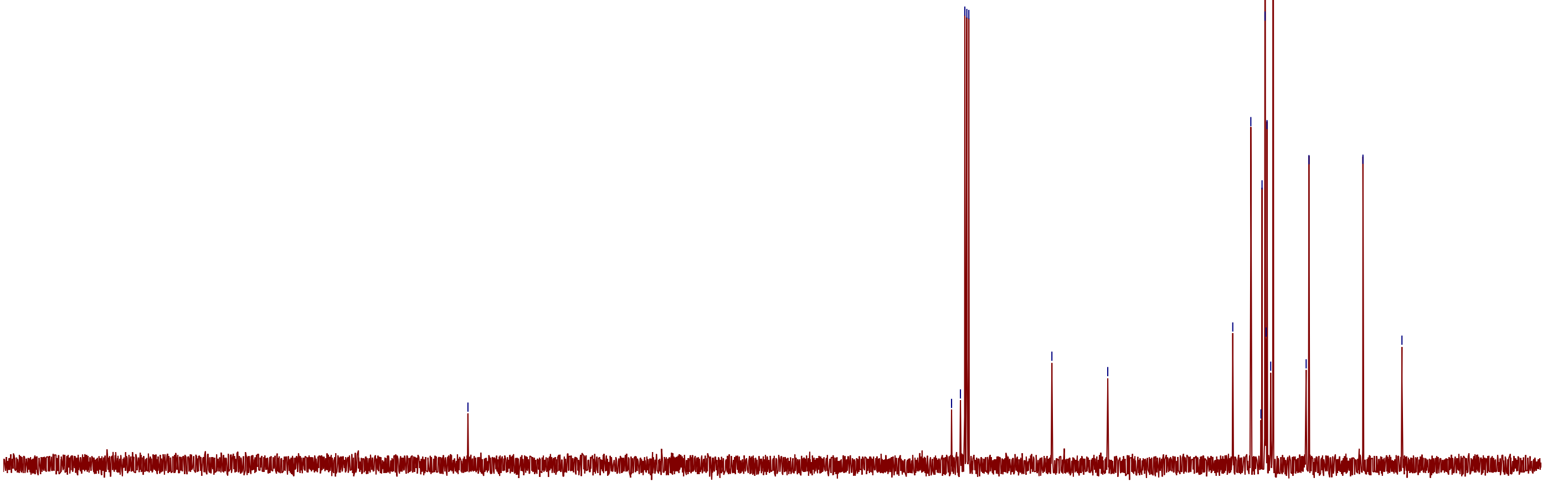




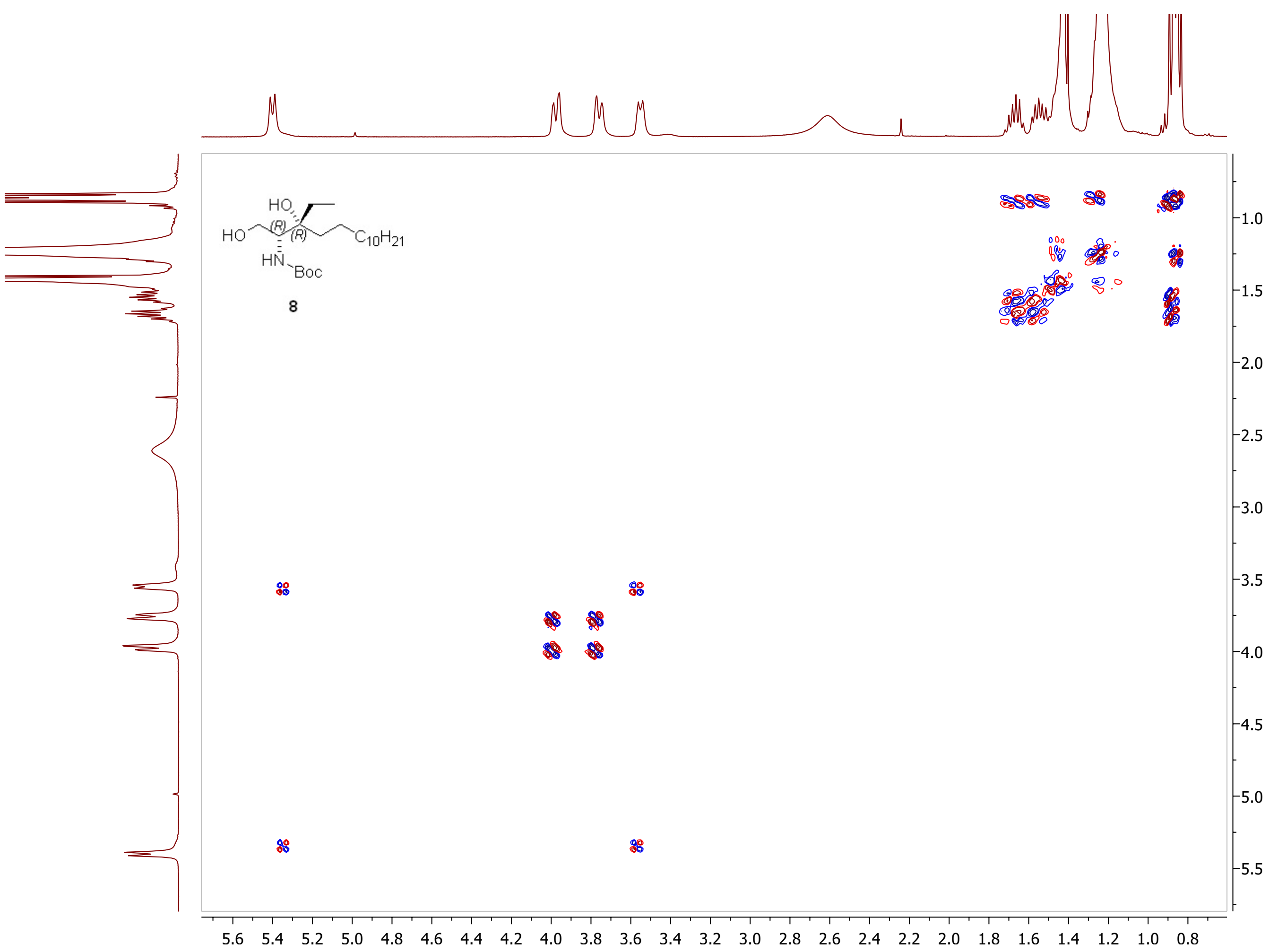




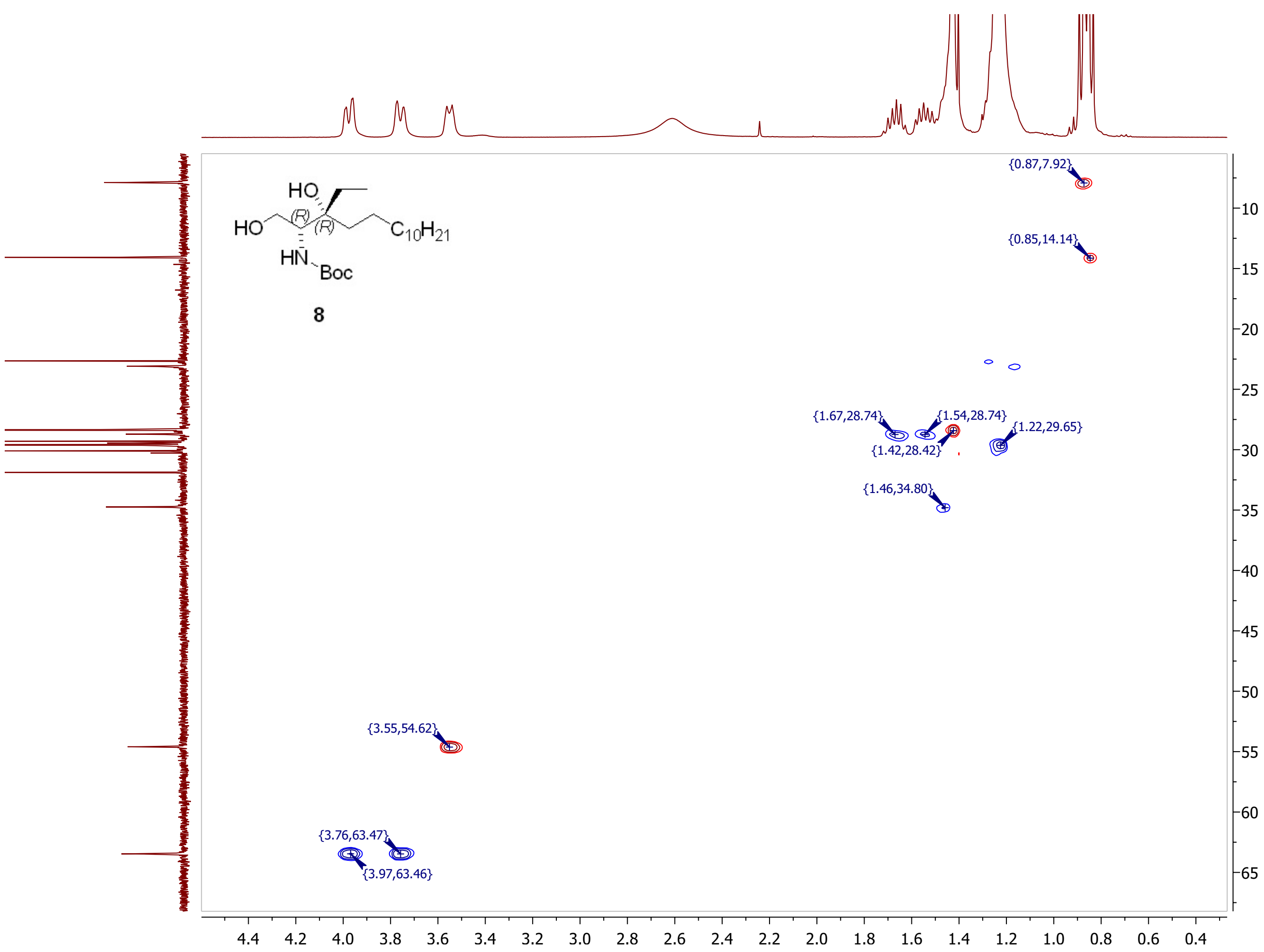




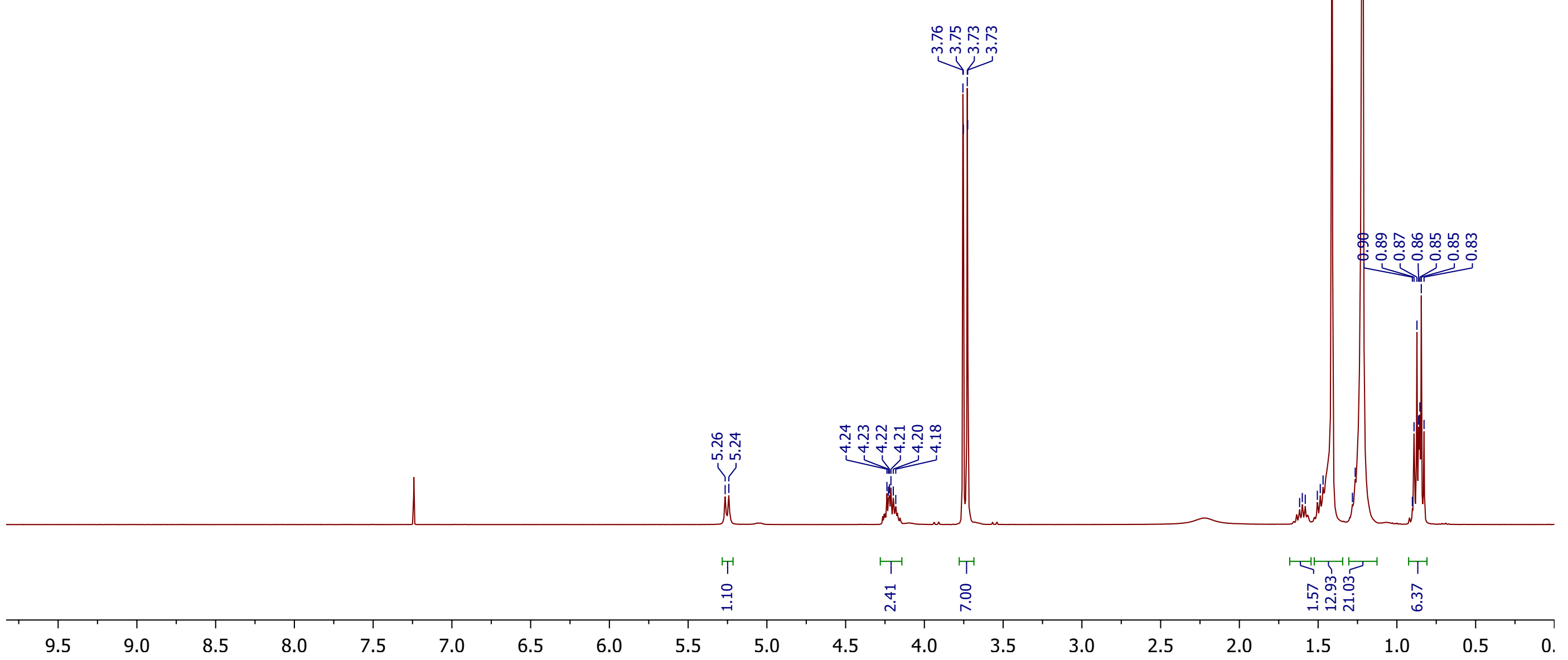




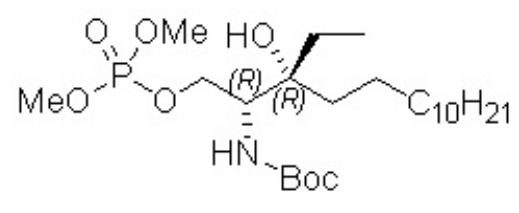

9

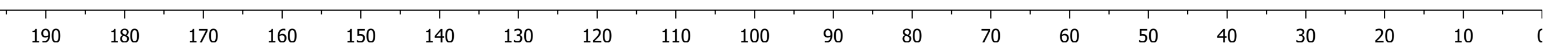




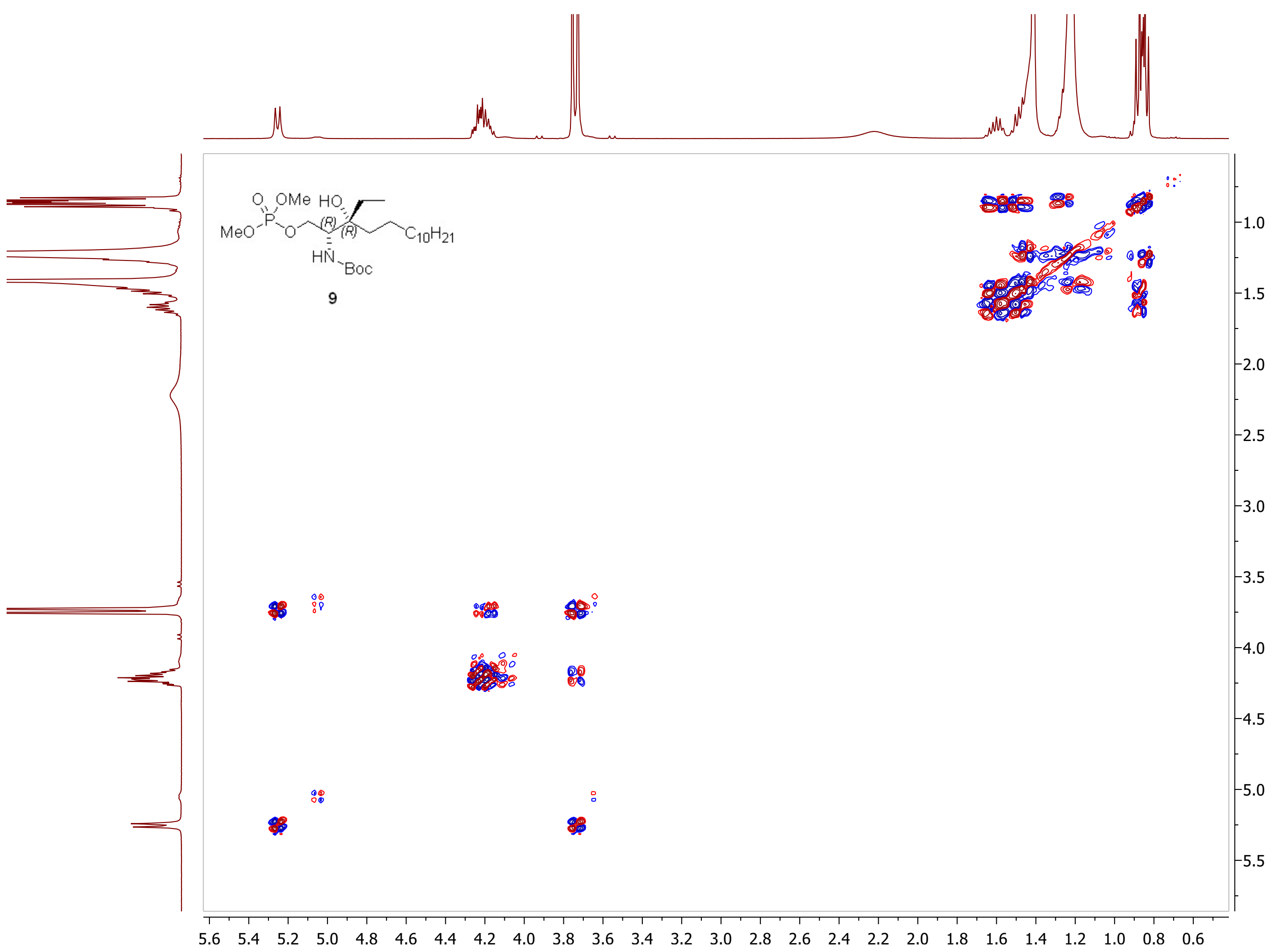




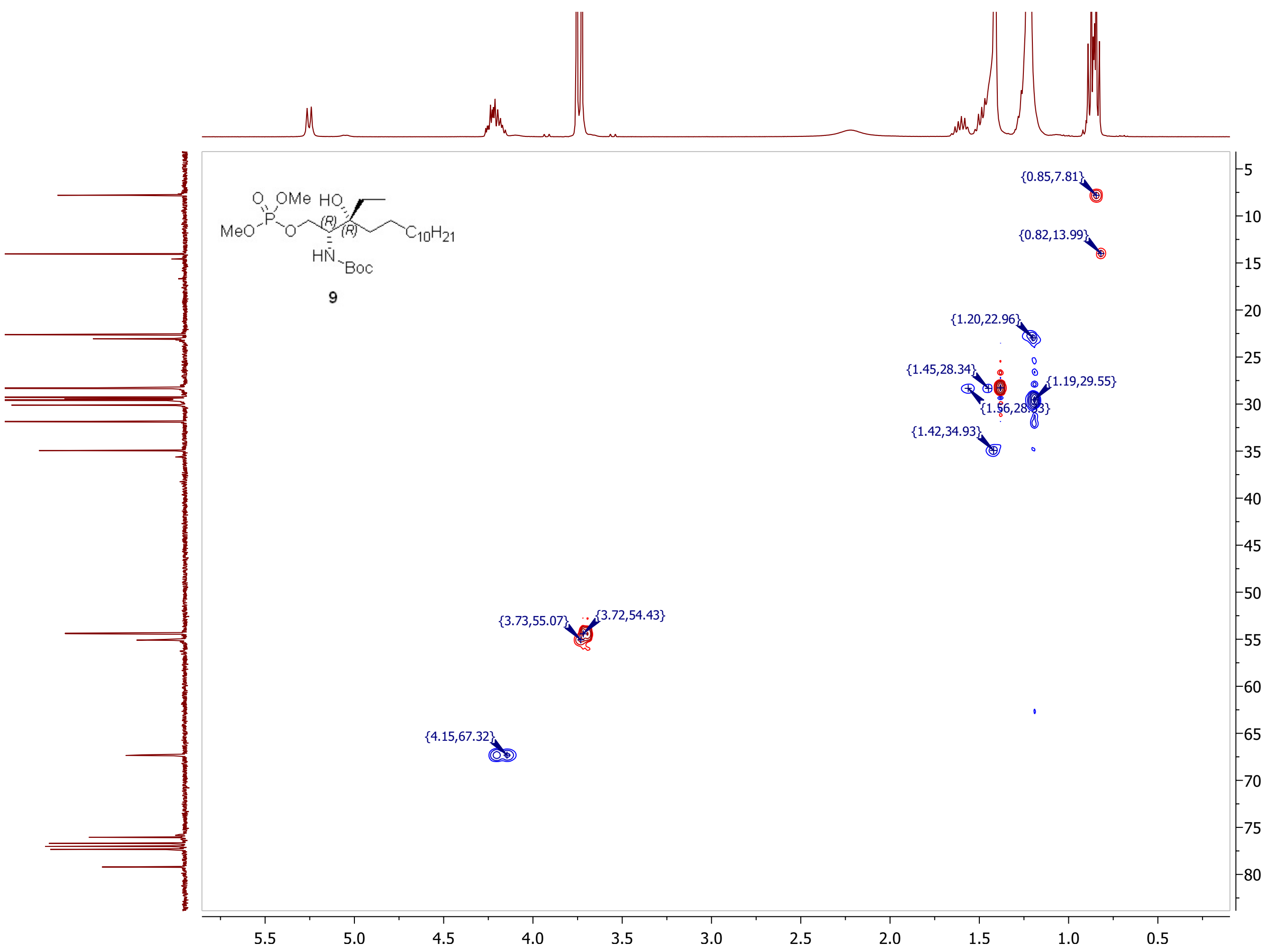



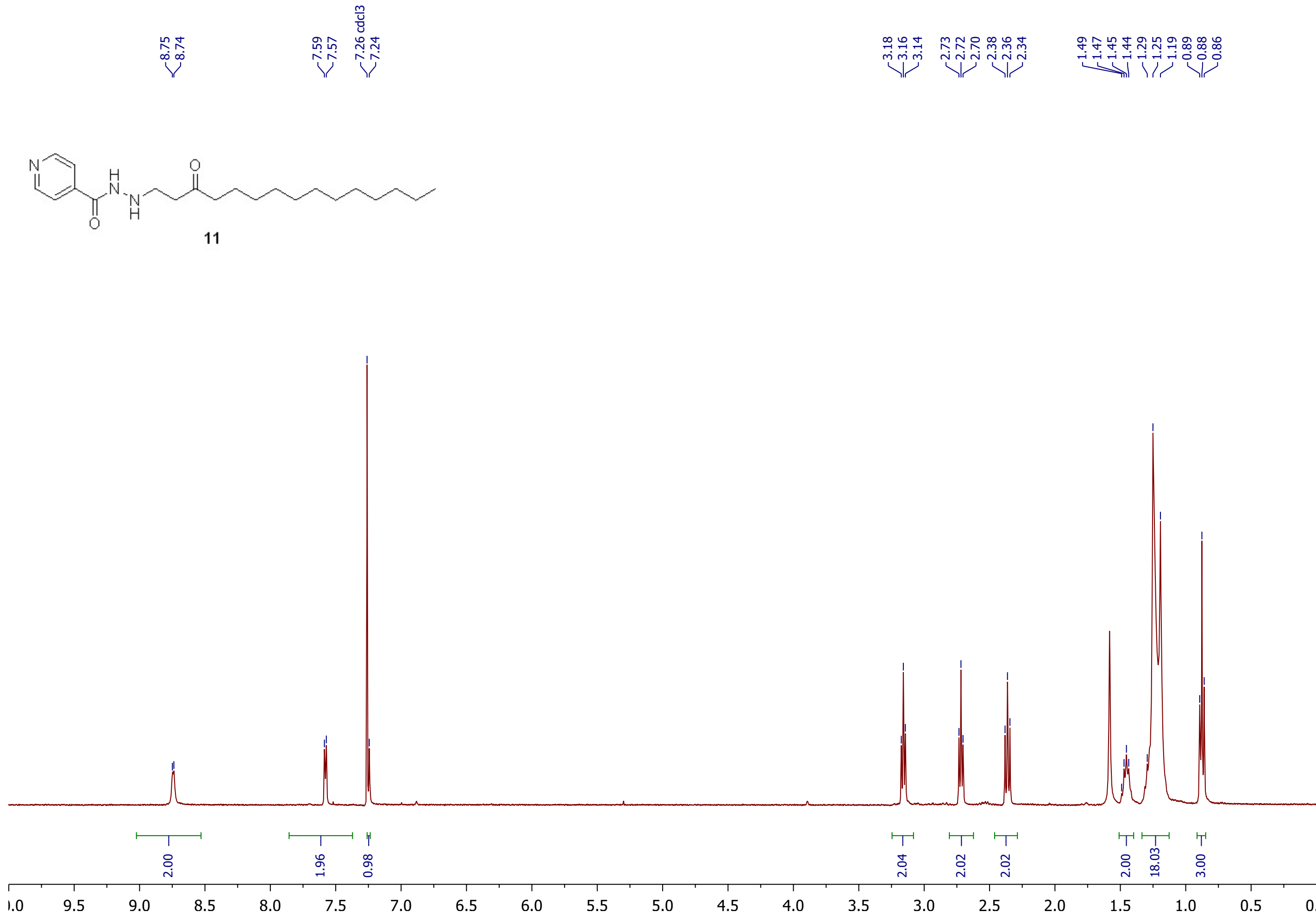


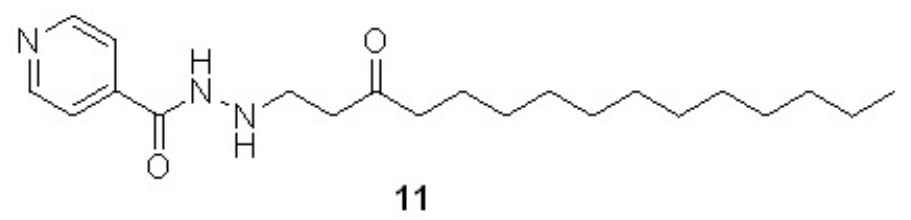




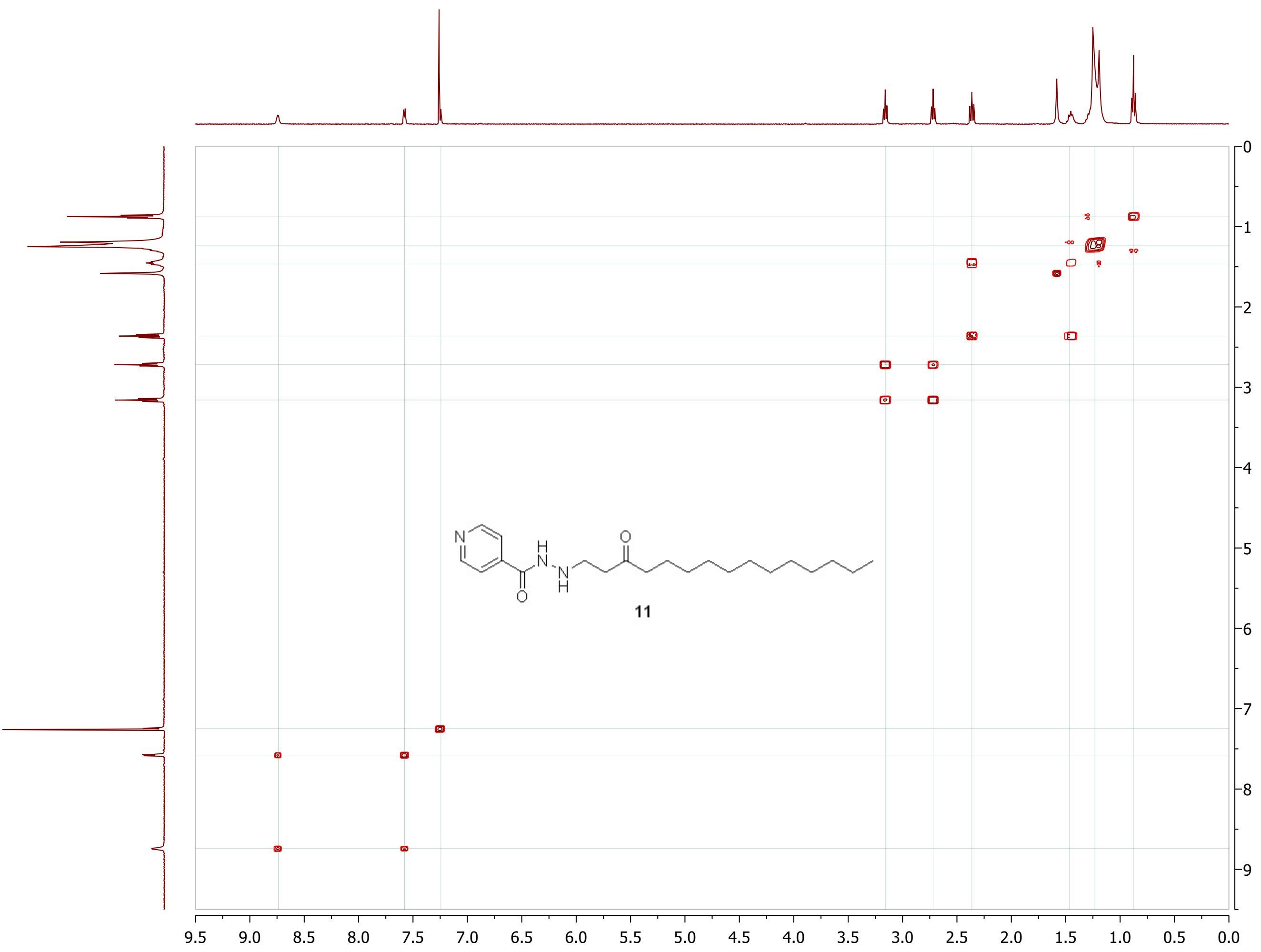




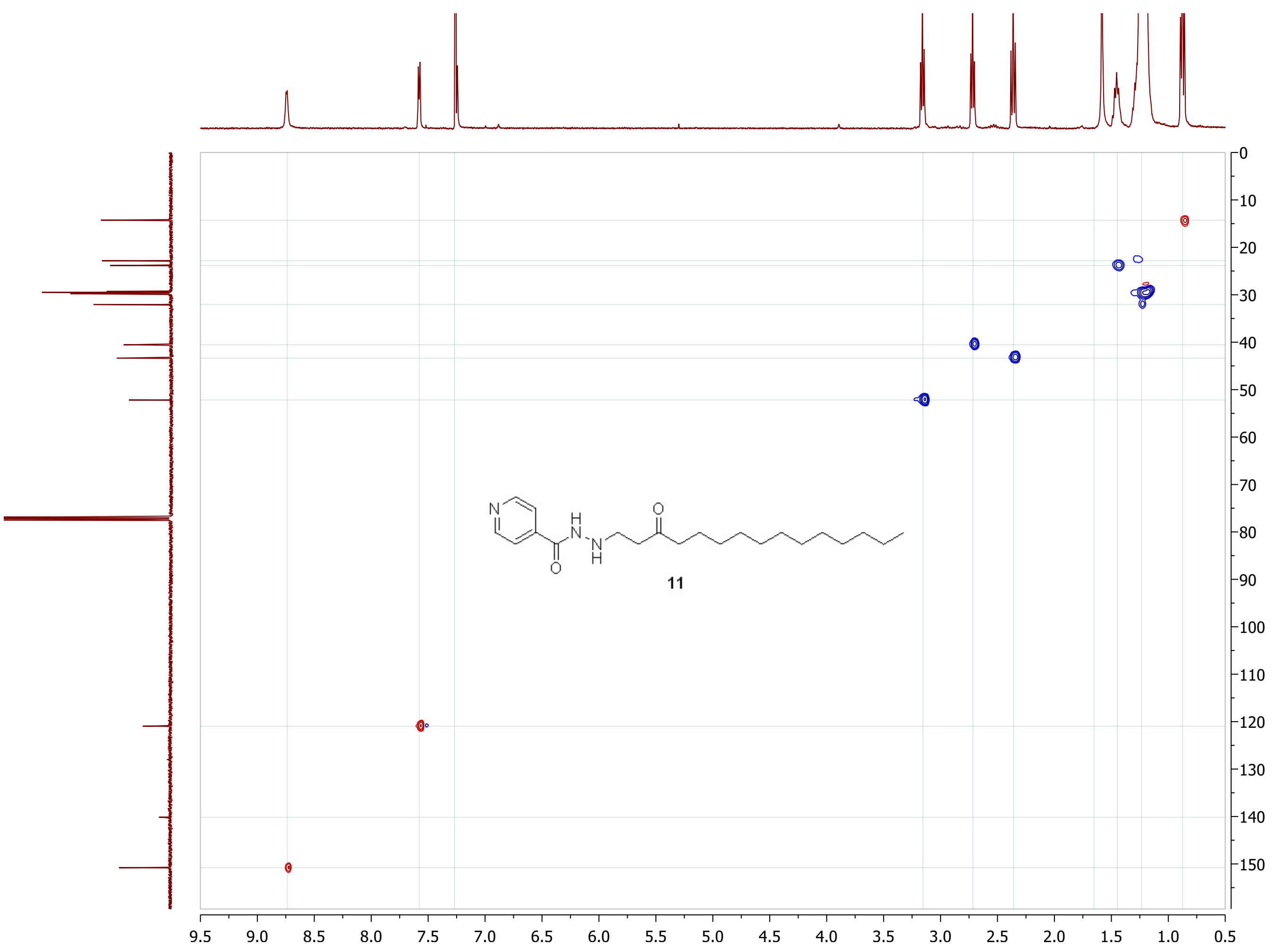


$\mathrm{HO}_{(\mathrm{S})} \mathrm{C}_{10} \mathrm{H}_{21}$

RBM10-1 (2S,3S)

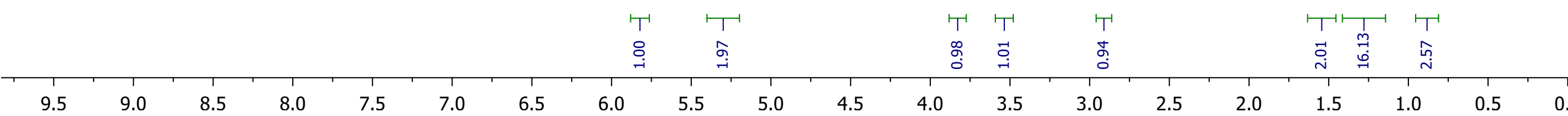


$\mathrm{C}_{10} \mathrm{H}_{21}$

RBM10-1 (2S,3S) 


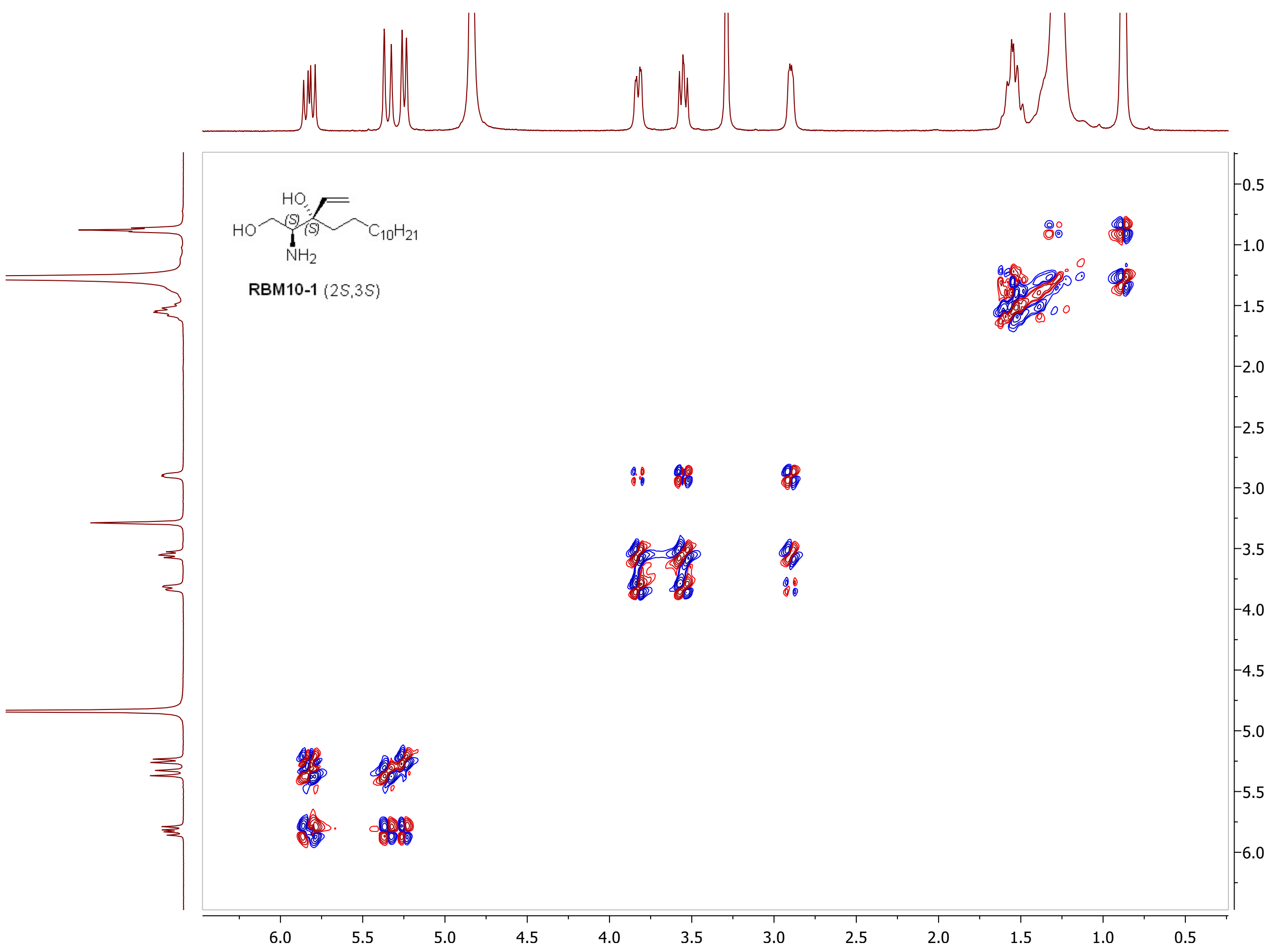




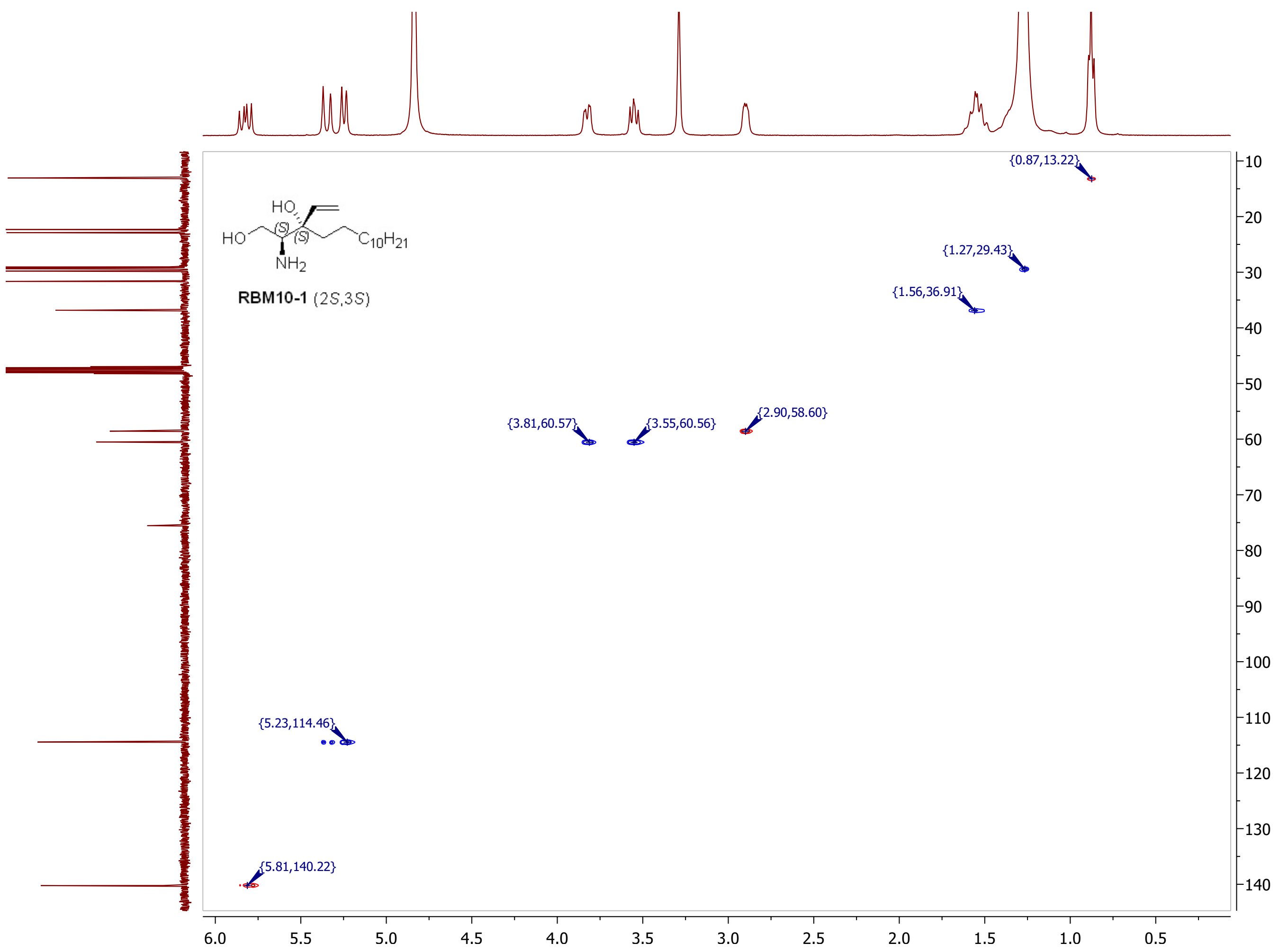




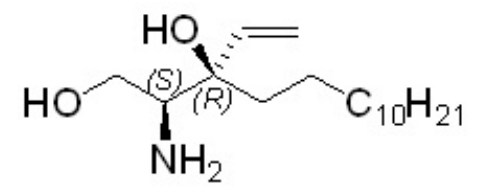

RBM10-2 (2S,3R)
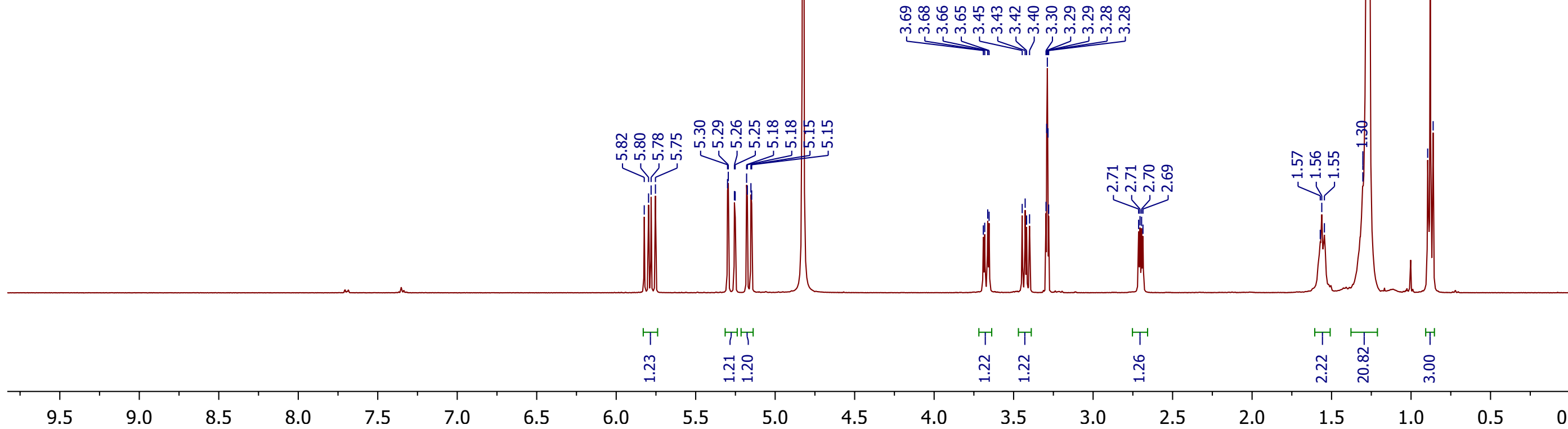


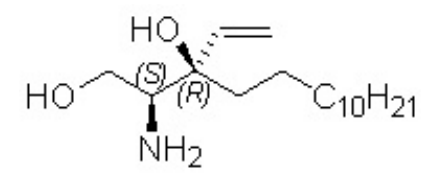

RBM10-2 $(2 S, 3 R)$

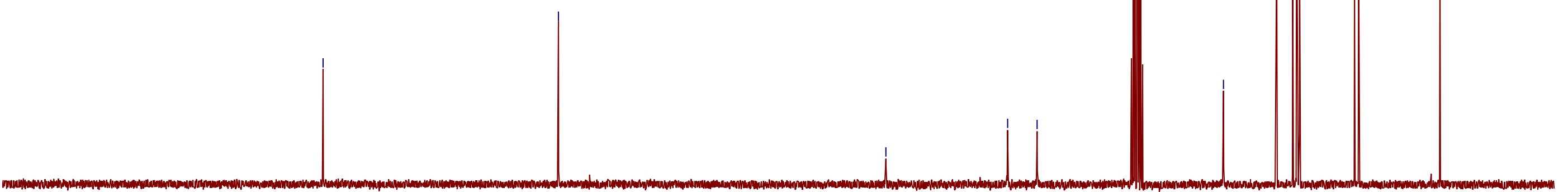




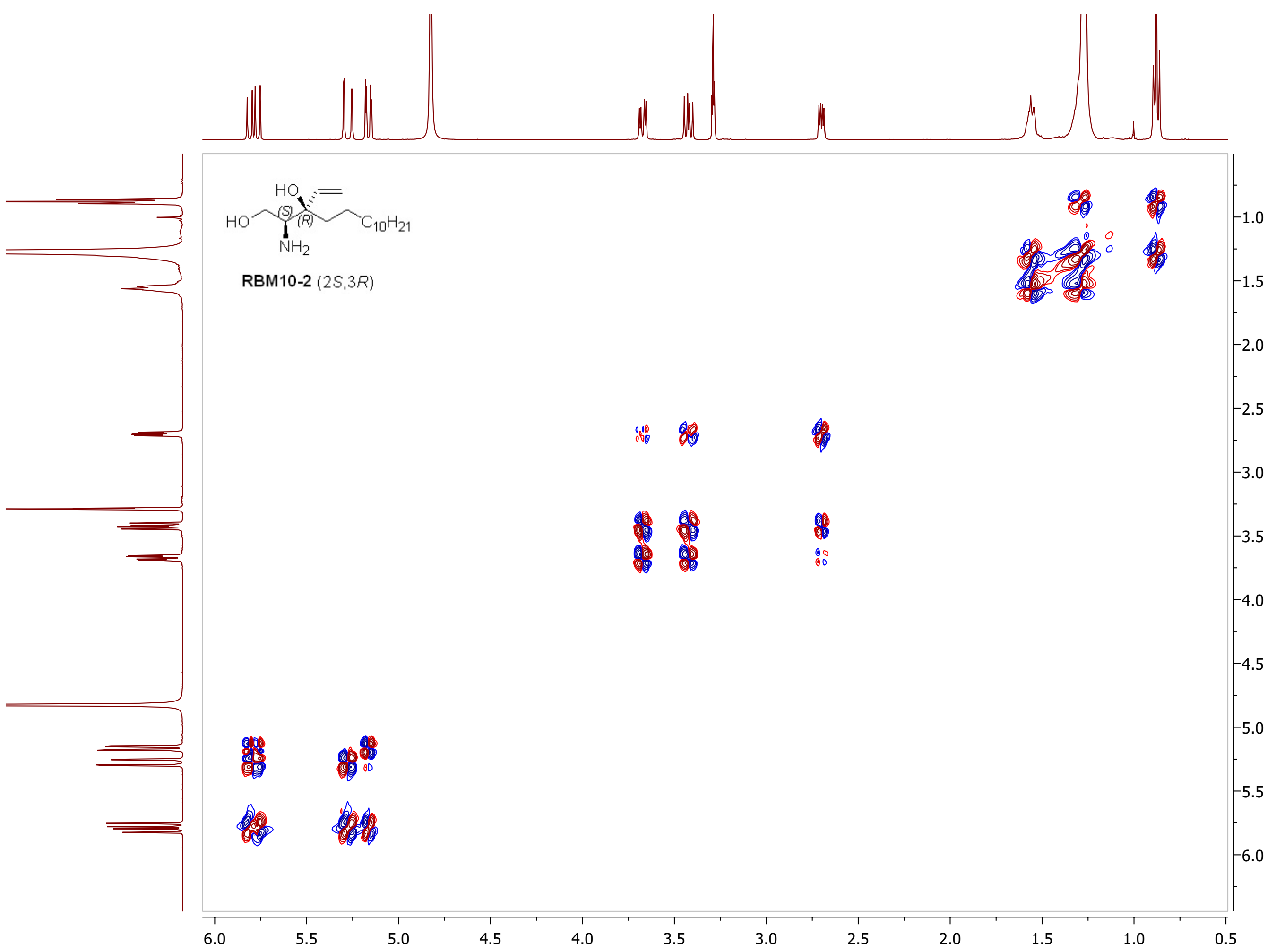




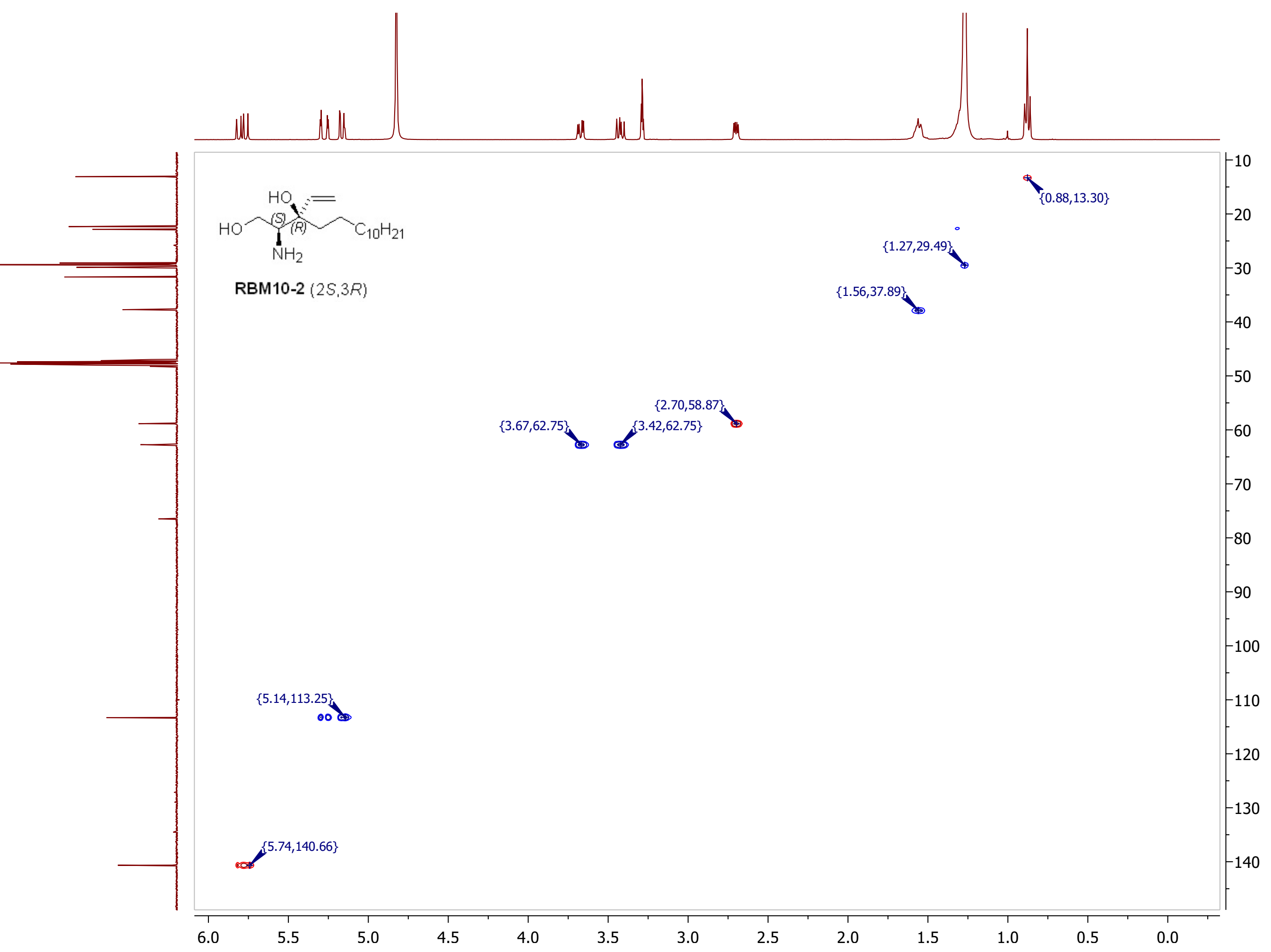




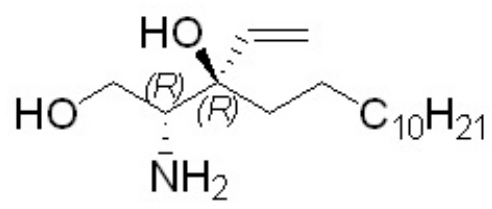

RBM10-3 $(2 R, 3 R)$

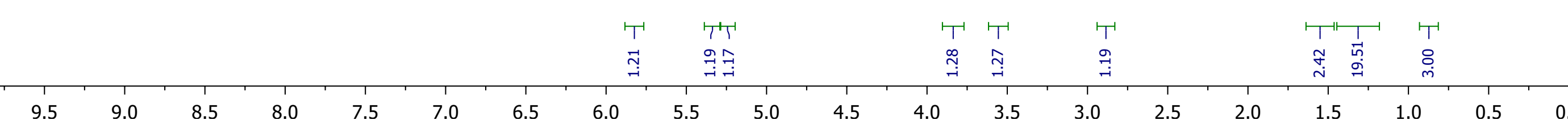




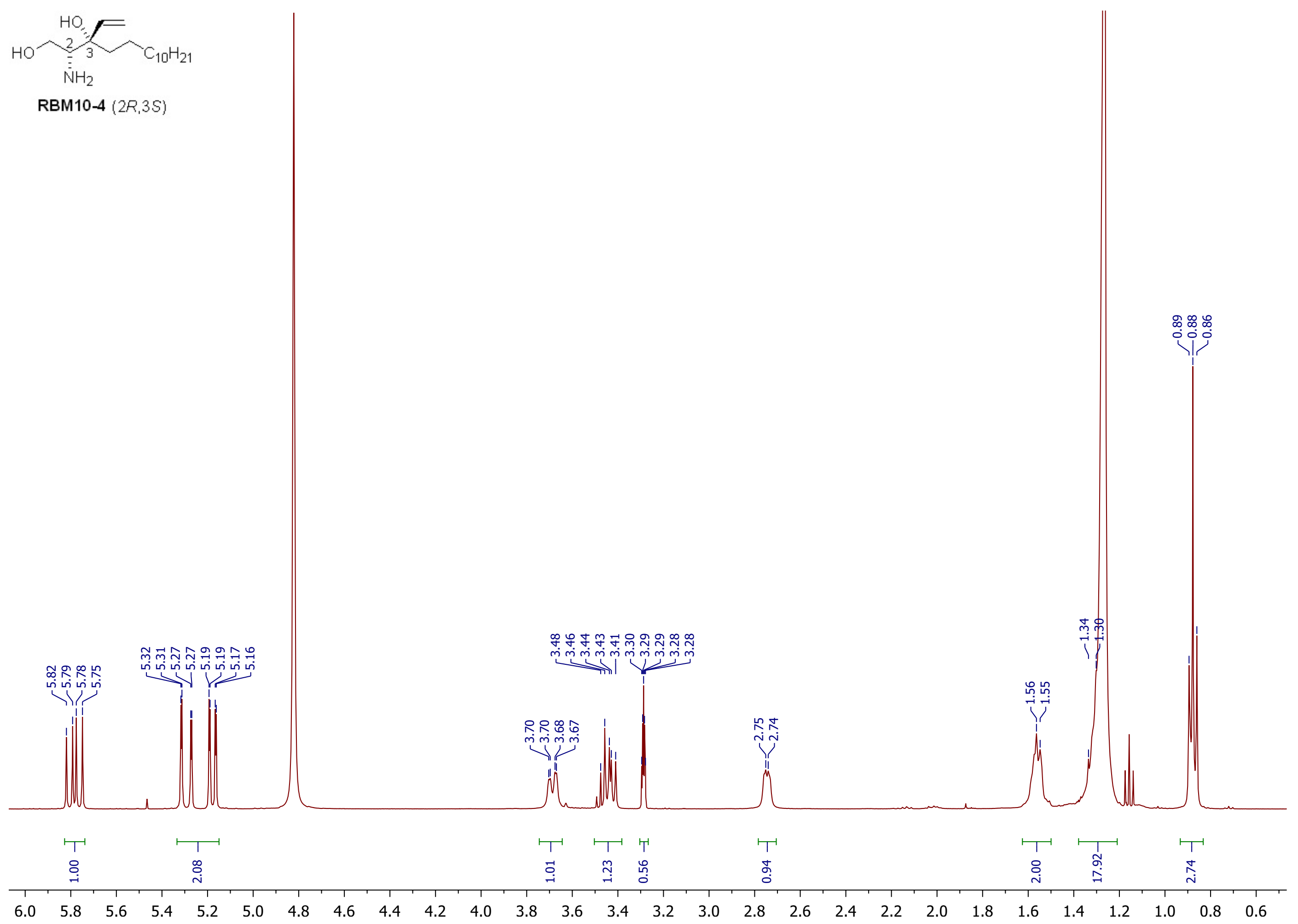


${ }_{\mathrm{HO}_{2}}$

RBM10-5 $(2 S, 3 S)$

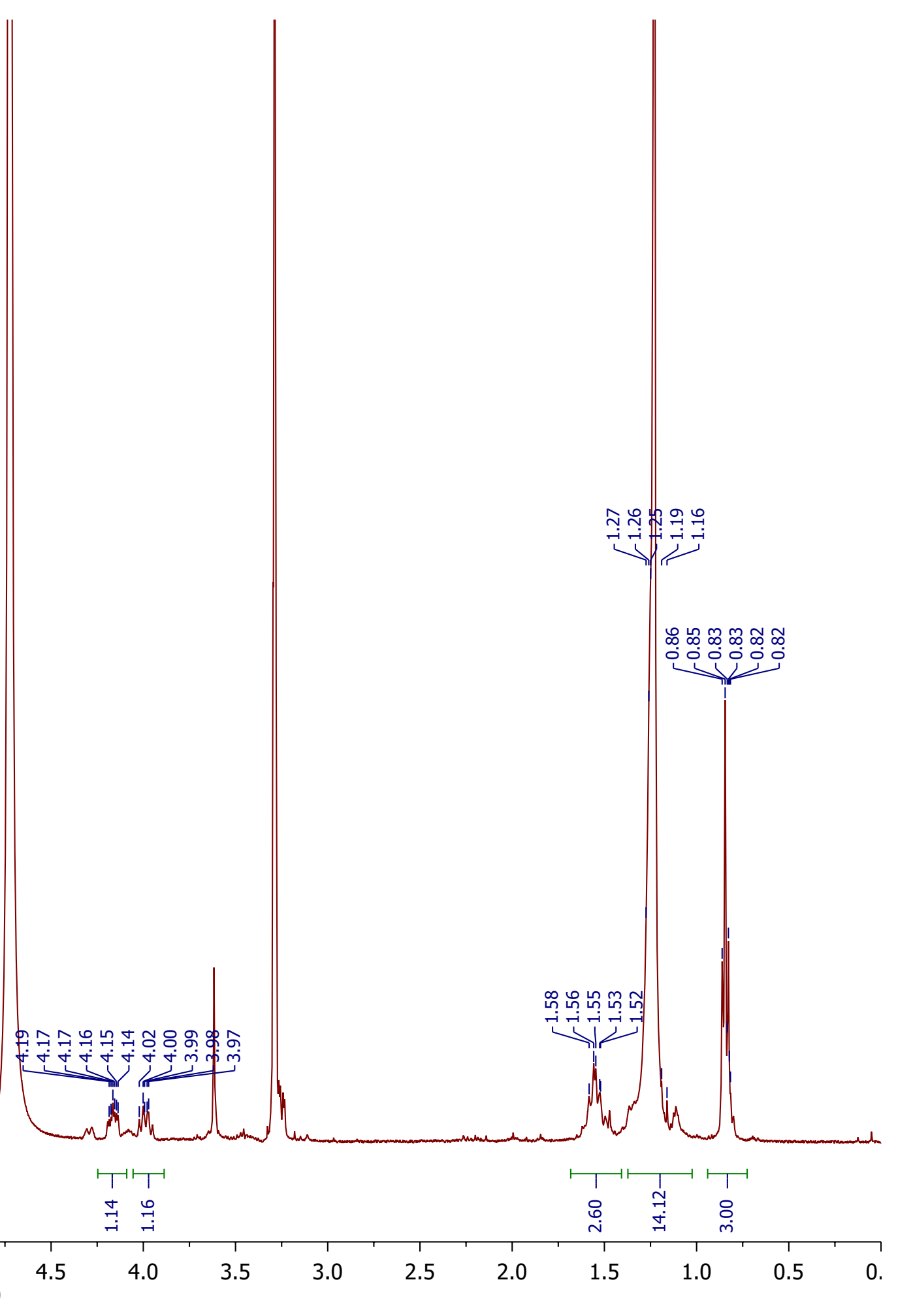




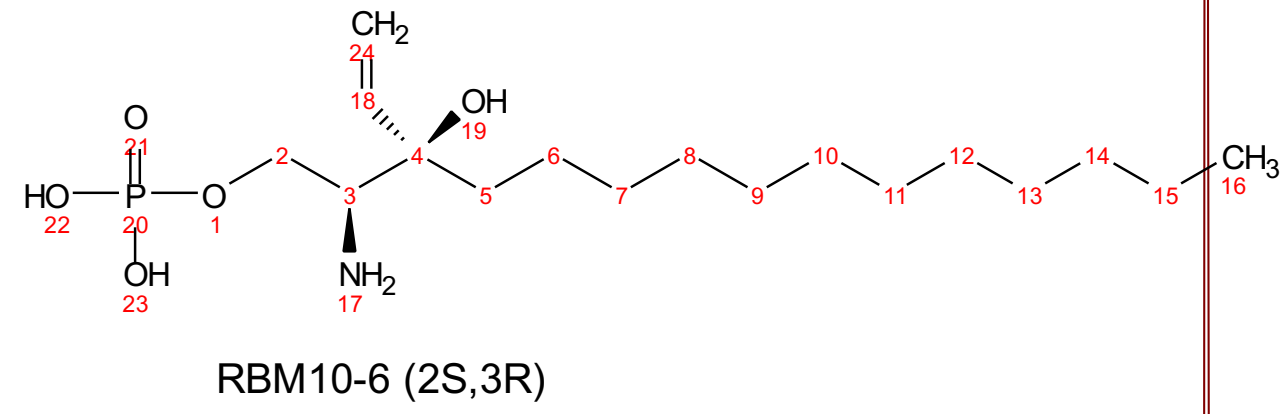


$\mathrm{HO}^{\mathrm{P}} \stackrel{\mathrm{P}}{\mathrm{O}} \mathrm{O}$

RBM10-7 $(2 R, 3 R)$

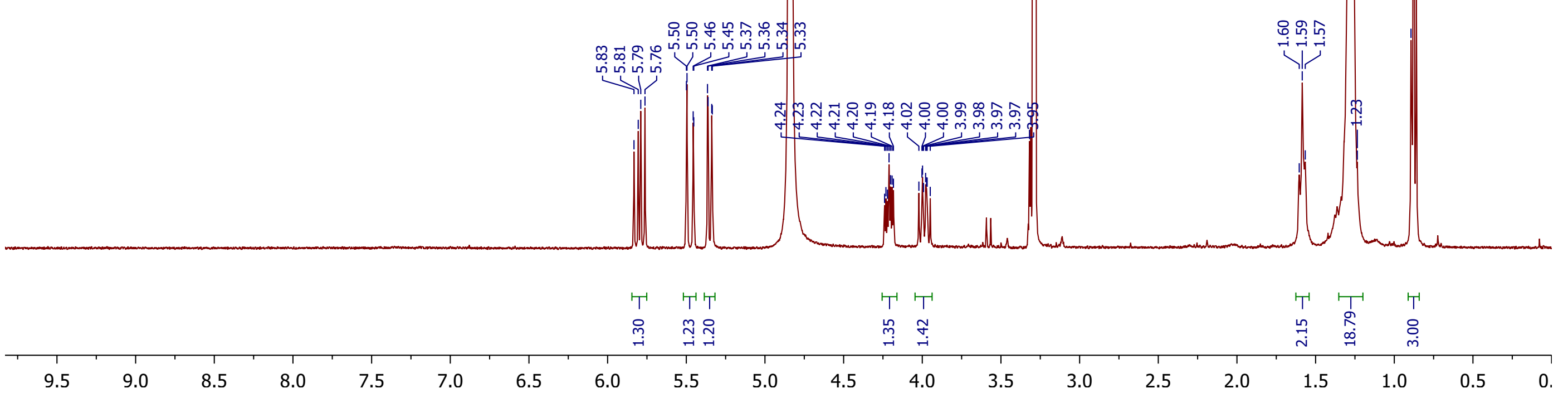




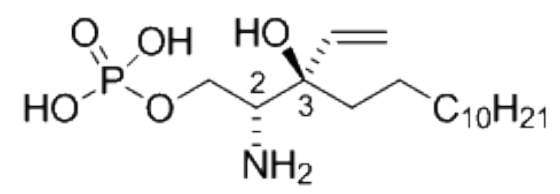

RBM10-7 $(2 R, 3 R)$

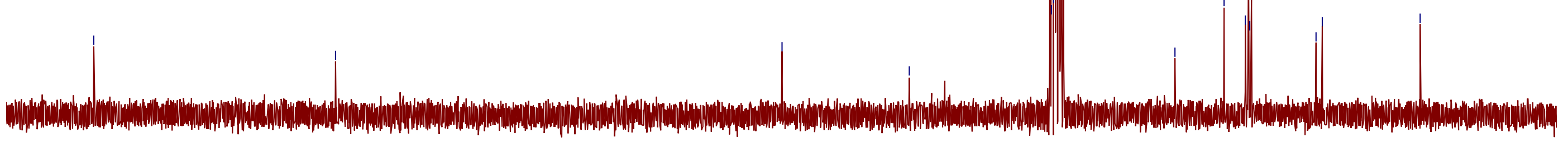




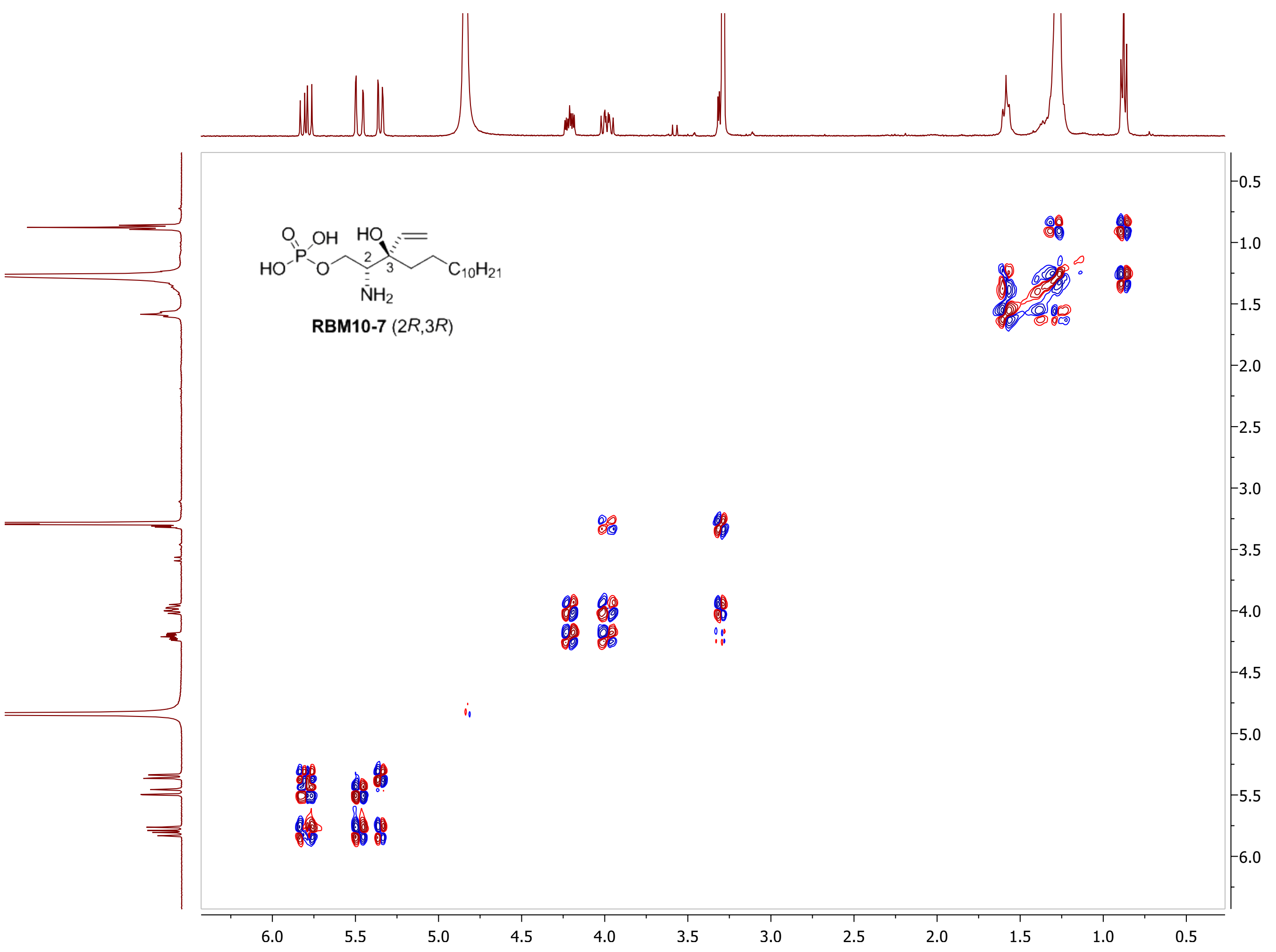


${ }_{\mathrm{C}_{10} \mathrm{H}_{21}}$

RBM10-8 $(2 R, 3 S)$

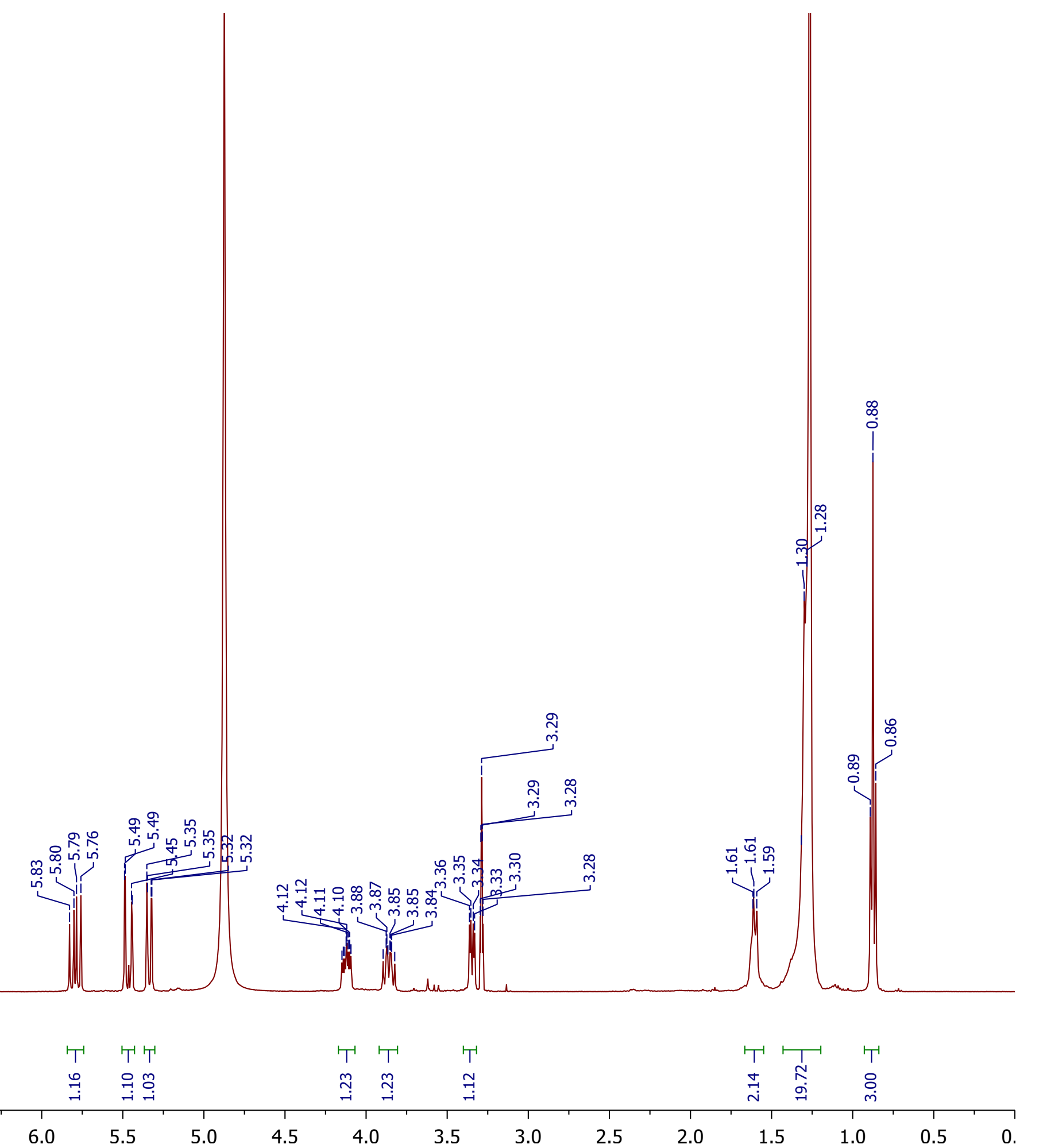


<smiles>C=C[C@](O)(CCC=O)[C@H](N)COP(=O)(O)O</smiles>

RBM10-8 $(2 R, 3 S)$ 


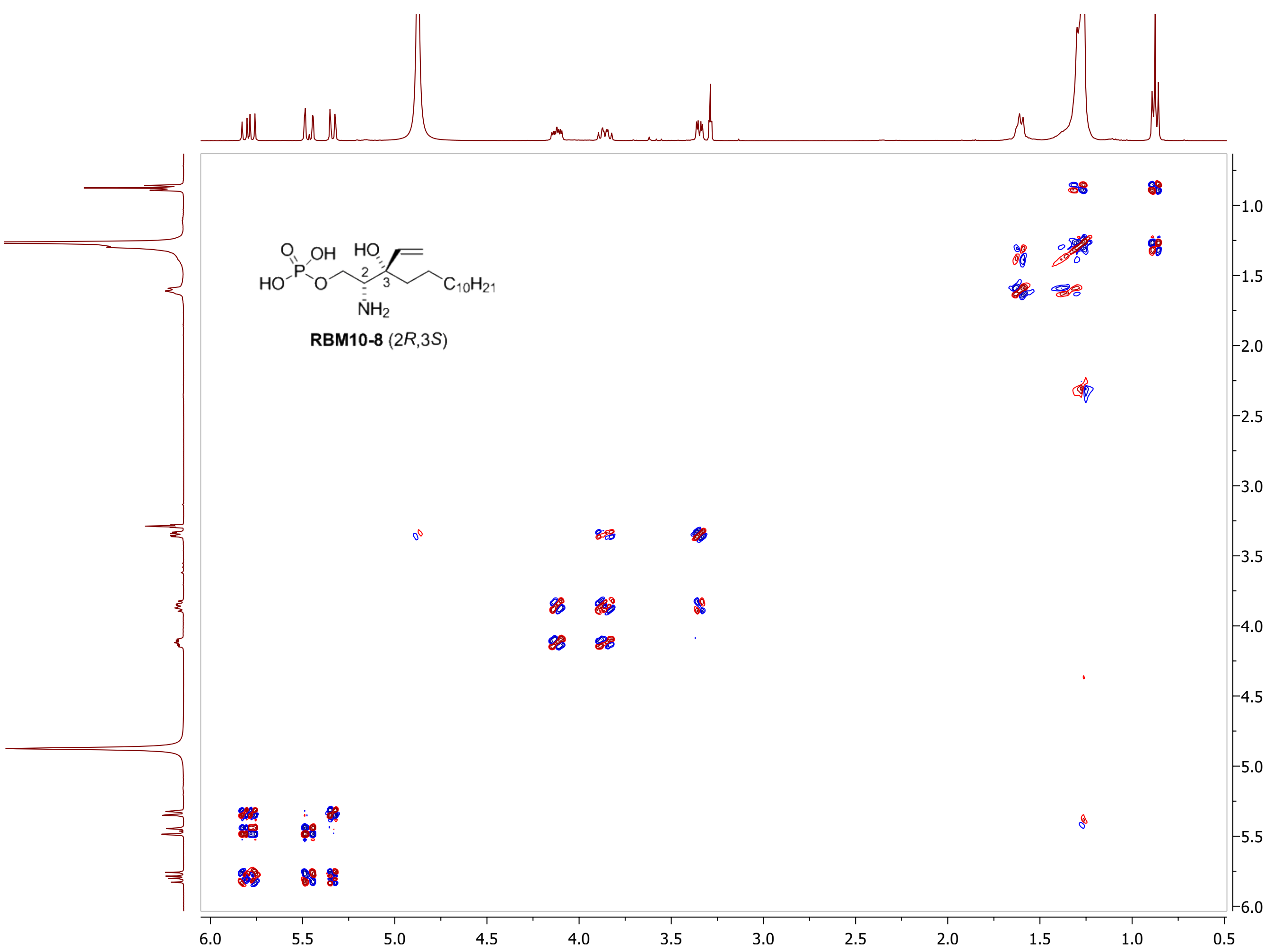




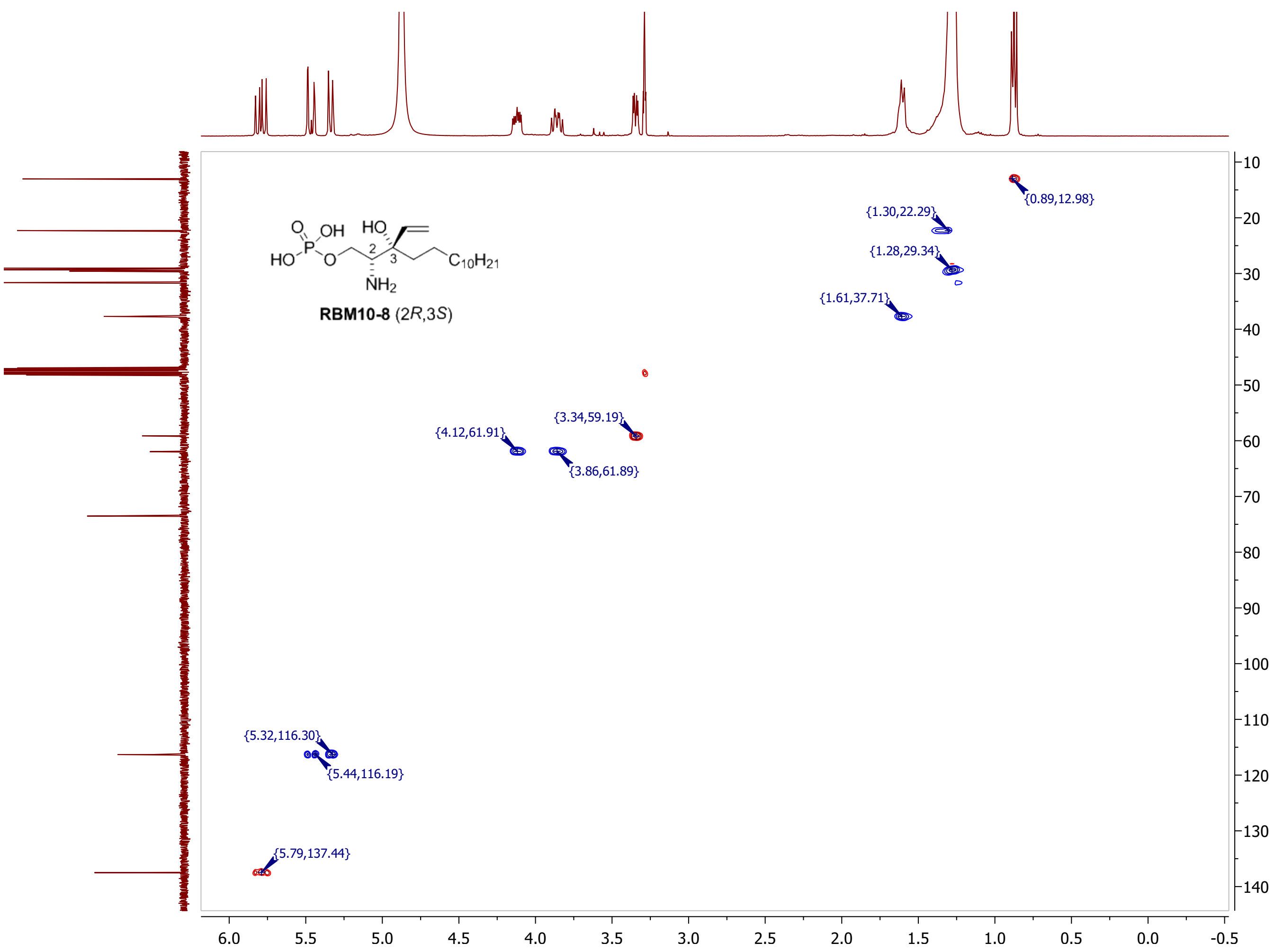




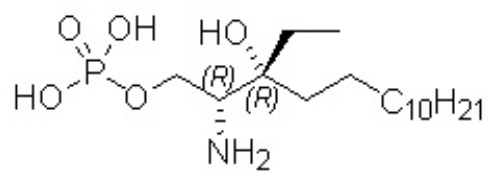

RBM10-9 $(2 R, 3 R)$

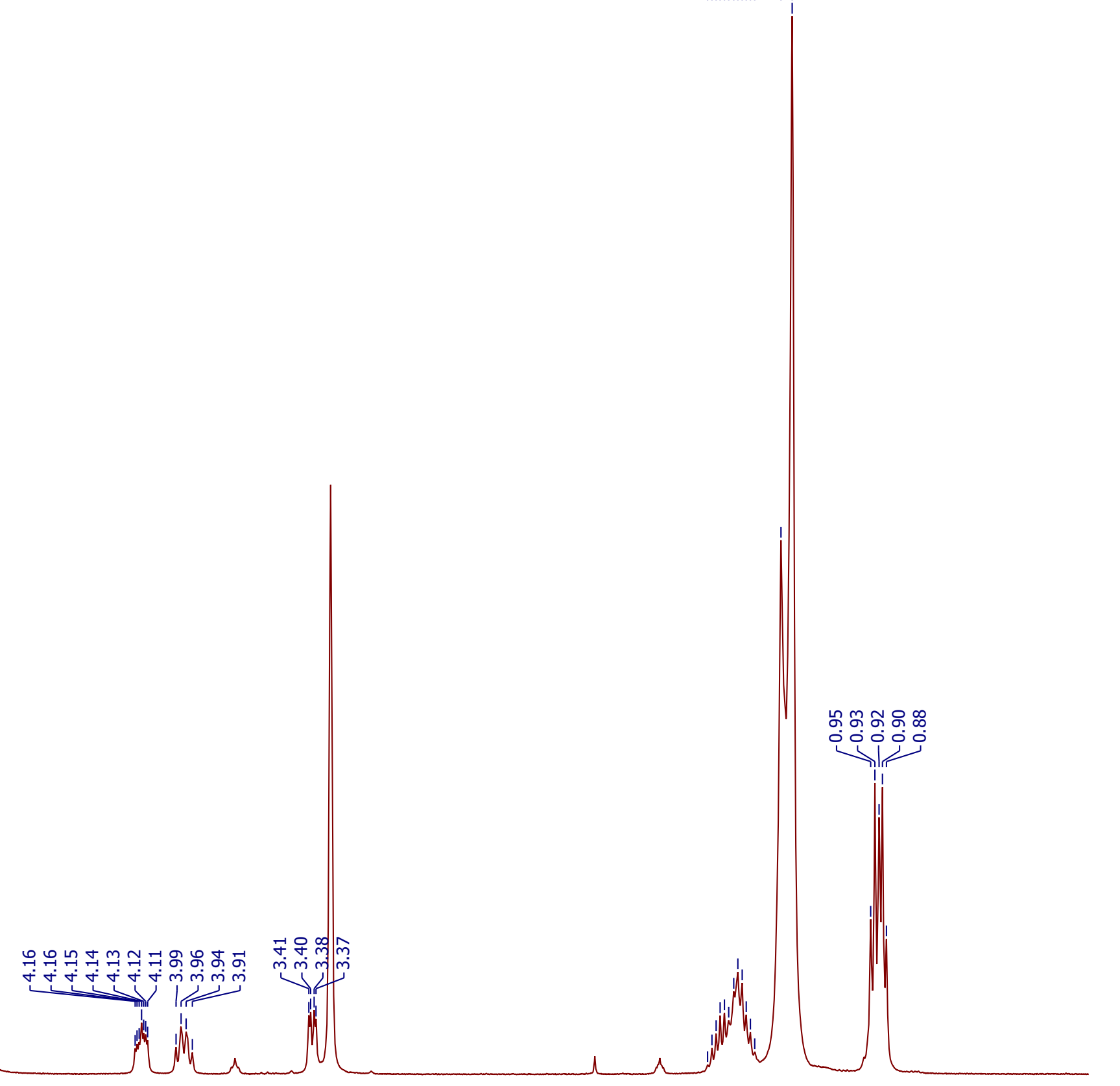

T $T$ T

$\stackrel{\sim}{\stackrel{\sim}{m}}$ $\stackrel{\text { กִ }}{i}$ 
$\mathrm{C}_{10} \mathrm{H}_{21} \quad$ RBM10-9 (2R,3R)

$\begin{array}{lllllllllllllllllllllllllllllllll}115 & 110 & 105 & 100 & 95 & 90 & 85 & 80 & 75 & 70 & 65 & 60 & 55 & 50 & 45 & 40 & 35 & 30 & 25 & 20 & 15 & 10 & 5 & \end{array}$




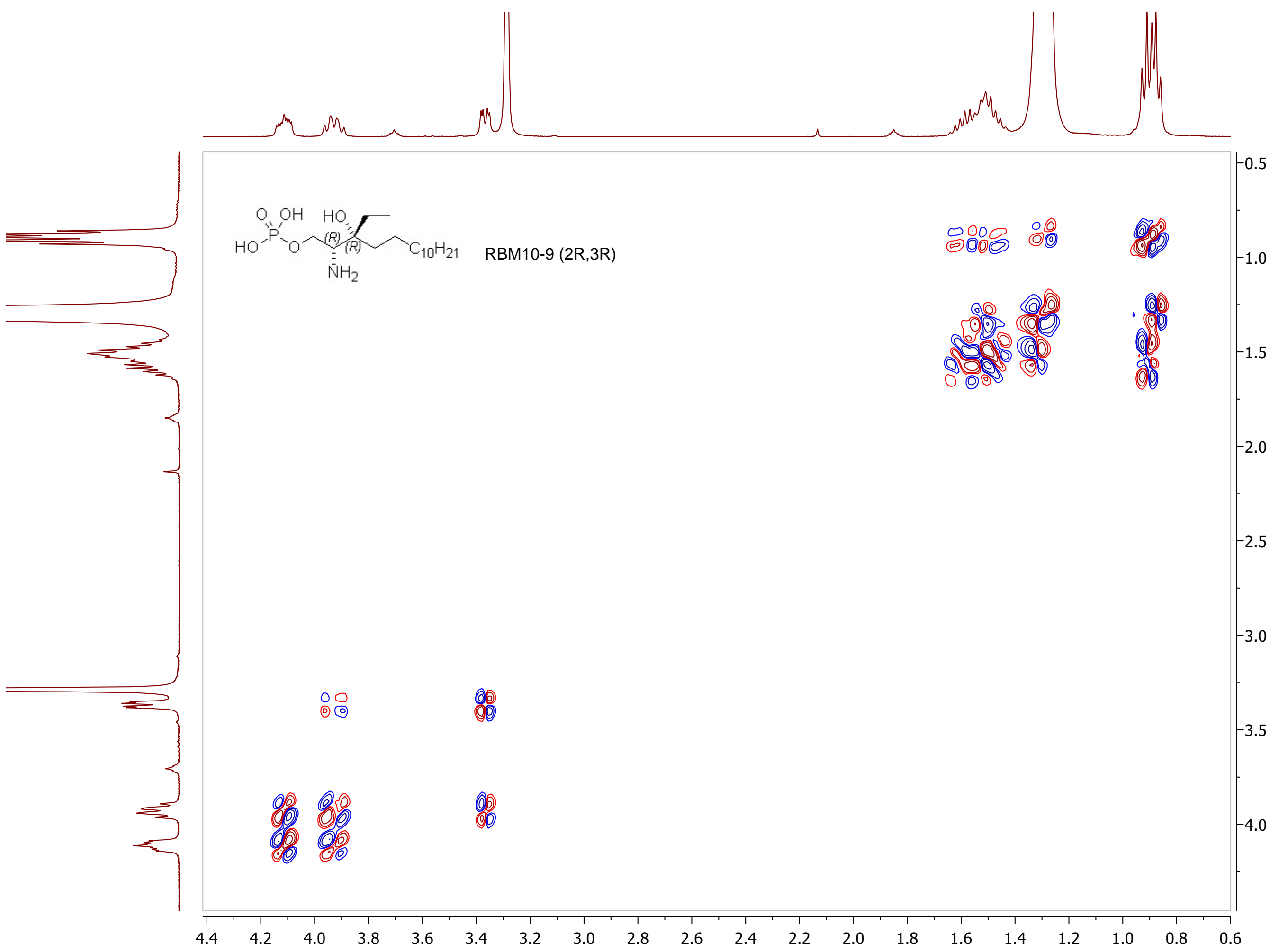




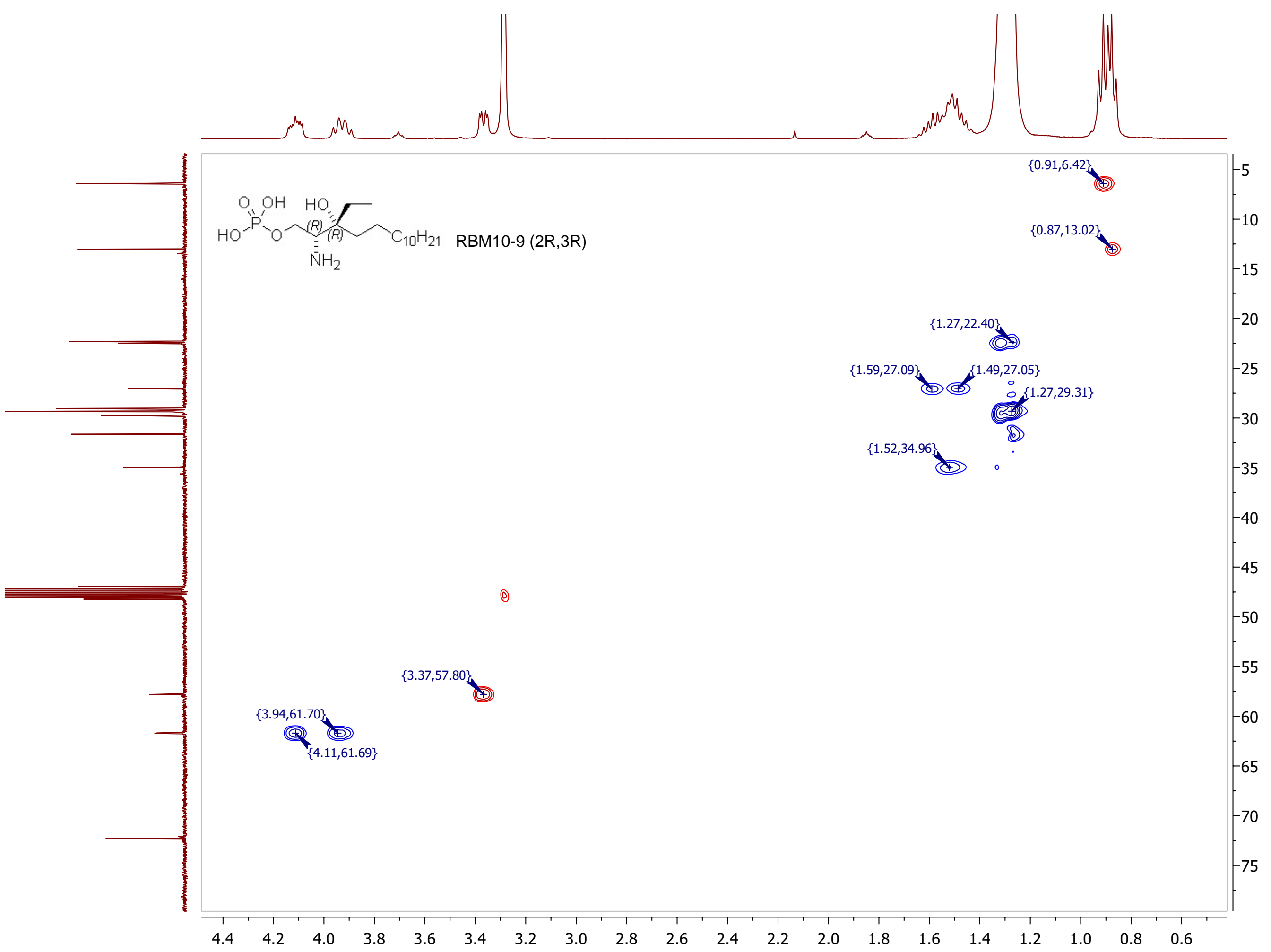

